\title{
Compactness in gauge theory
}

\author{
Patrícia Muñoz Ewald
}

\author{
Dissertation submitted to the \\ Institute of Mathematics and \\ Statistics of the University of São \\ Paulo for the degree of \\ Master of Science
}

Program: Mathematics

Advisor: Prof. Dr. Cristian Ortiz

During the development of this dissertation the author was financially supported by CNPq

São Paulo, June 2021 


\section{Compactness in gauge theory}

This is the original version of the dissertation developed by the candidate Patrícia Muñoz Ewald as it was submitted to the Judging

Committee. 
Ao meu Tata. 


\begin{abstract}
In 1982, Karen Uhlenbeck published two foundational papers in gauge theory, which quickly led to Simon Donaldson's Fields medal winning result on topology of four-manifolds, and to the beginning of an era of using gauge theoretic techniques as tools for proving theorems. In 2019, she became the first (and thus far only) woman to receive the Abel prize, for these and other groundbreaking works in geometric analysis.

In one of these works, entitled Connections with $L^{p}$ bounds on curvature, Uhlenbeck proved two very important technical results on the existence of a good gauge, and the sequential compactness of weak connections with bounded curvature. In this work, we prove these results and then address their immediate consequence: the uniform convergence of weak Yang-Mills connections with bounded curvature.
\end{abstract}

Keywords: gauge theory, geometric analysis, fibre bundles, connections, Uhlenbeck compactness, Yang-Mills equation.

\title{
Resumo
}

Em 1982, Karen Uhlenbeck publicou dois artigos fundamentais em teoria de gauge, que rapidamente levaram Simon Donaldson ao resultado em topologia de 4-variedades que lhe rendeu a medalha Fields, e ao início de uma era de utilização de técnicas de teoria de gauge em demonstrações. Em 2019, se tornou a primeira (e por enquanto, única) mulher a receber o prêmio Abel, por esses e outros trabalhos revolucionários em análise geométrica.

Em um desses trabalhos, Connections with $L^{p}$ bounds on curvature, Uhlenbeck provou dois resultados técnicos muito importantes sobre a existência de uma boa escolha de gauge, e sobre a compacidade sequencial de conexões fracas com curvatura uniformemente limitada. Neste trabalho, provamos esses resultados e em seguida nos voltamos à uma consequência imediata: a convergência uniforme de sequências de conexões Yang-Mills fracas com curvatura uniformemente limitada.

Palavras-chave: teoria de gauge, análise geométrica, fibrados, conexões, compacidade de Uhlenbeck, equação de Yang-Mills. 



\section{Contents}

$\begin{array}{lll}1 & \text { Gauge theory } & 1\end{array}$

1.1 Vector bundles . . . . . . . . . . . . . . . . . . . . . . 1

1.1.1 Riemannian manifolds . . . . . . . . . . . . . . . . . . 5

1.2 Principal bundles . . . . . . . . . . . . . . . . . . . . 7

1.2 .1 Local formulations and results $\ldots \ldots \ldots \ldots$. . . . . . . . . 14

1.2 .2 Several results on $G, \mathfrak{g}$ and the action on $P \ldots \ldots$. . . . . 17

1.3 An analytic interlude . . . . . . . . . . . . . . . . . . . . . . . 19

1.4 Analysis in gauge theory $\ldots \ldots \ldots \ldots . \ldots \ldots 25$

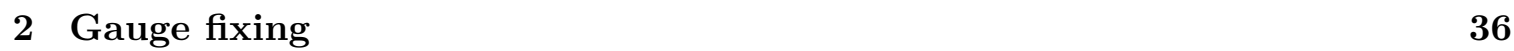

2.1 Motivation and main result $\ldots \ldots \ldots \ldots \ldots$. . . . . . . . . . . . . . . . . . . .

$2.2 \quad$ Proof $\ldots \ldots \ldots \ldots \ldots \ldots$

3 Compactness $\quad \mathbf{5 2}$

3.1 Weak compactness . . . . . . . . . . . . . . . . . . . . . . 54

3.2 Strong compactness $\ldots \ldots \ldots \ldots \ldots \ldots \ldots \ldots$

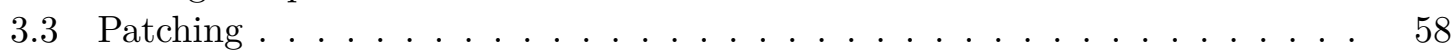

\begin{tabular}{|ll}
\hline A Background material & 64
\end{tabular}

\begin{tabular}{lr}
\hline Bibliography & 68
\end{tabular} 


\section{Chapter 1}

\section{GAUGe THEORY}

Gauge theory is essentially the study of connections on principal bundles. The goal of this chapter is to expand on this, by first giving an introduction to the theory of vector and principal bundles, and giving the definitions of connections and gauge transformations, first assuming that all objects are smooth. Then, in section 1.3, we introduce Sobolev spaces of sections of fibre bundles and state the results that will be used extensively throughout this work. Finally, in section 1.4, we define the Sobolev spaces of connections and gauge transformations and prove several lemmas.

\subsection{Vector bundles}

In this first short section we define vector bundles and connections. At the end of the section we especialize to the case of Riemannian manifolds in order to introduce notation and objects which will be used extensively throughout the rest of the text. For an introduction to this subject see e.g. [Cra15.

Definition 1.1. A vector bundle of rank $r$ over a manifold $M$ consists of

- a manifold $E$,

- a surjective map $\pi: E \rightarrow M$, and

- for each $x \in M$, a vector space structure on the fibres $E_{x}:=\pi^{-1}(x)$,

satisfying a local triviality condition: around each $x \in M$ there is a neighbourhood $U$ and a diffeomorphism

$$
\phi:\left.E\right|_{U}:=\pi^{-1}(U) \rightarrow U \times \mathbb{K}^{r}
$$

sending each fibre $E_{x}$ isomorphically to $\{x\} \times \mathbb{K}^{r}$, for $\mathbb{K}=\mathbb{R}$ or $\mathbb{C}$.

A section of the vector bundle is a map $s: M \rightarrow E$ such that $\pi \circ s(x)=x$, that is, it takes points on $M$ to the corresponding fibre. It is a smooth section if $s$ is a smooth map between manifolds, and we denote the space of sections of a vector bundle $E$ by $\Gamma(E)$; it is easily seen to be a vector space over $\mathbb{K}$ with the operations defined pointwise, and in fact it is a module over $C^{\infty}(M)$.

An important notion is that of a trivial vector bundle. The (real or complex) trivial vector bundle of rank $r$ over $M$ is simply $\pi: M \times \mathbb{K}^{r} \rightarrow M$, where $\pi$ is the projection on the first factor. A vector bundle is said to be trivializable if it is isomorphic to the 
corresponding trivial bundle. A vector bundle morphism 1 between two vector bundles $E$ and $F$ over $M$ is a smooth map $u: E \rightarrow F$ such that it preserves the fibres and the restriction $u_{x}: E_{x} \rightarrow F_{x}$ is linear; it is an isomorphism when $u$ is a diffeomorphism, or equivalently when the $u_{x}$ are linear isomorphisms.

Just as manifolds have local coordinate charts which make them more tractable using tools from calculus, vector bundles have frames. A frame for a rank $r$ vector bundle is a collection of $r$ sections,

$$
e=\left(e^{1}, \ldots, e^{r}\right)
$$

such that for each $x \in M, e^{1}(x), \ldots, e^{r}(x)$ is a basis for the vector space $E_{x}$. Vector bundles may not have globally defined frames, and indeed a choice of frame is equivalent to a trivialization (a choice of isomorphism to the trivial bundle). Nevertheless, local frames always exist: the local triviality condition in the definition is equivalent to the existence of a local frame around every point in $M$.

New vector bundles can be constructed from previously known ones. Any operation that is natural in the context of vector spaces (e.g., direct sum, dual, tensor product) extends to vector bundles. For instance, if $E \rightarrow M$ is a vector bundle, we can define its dual $E^{*} \rightarrow M$ by defining

$$
E_{x}^{*}=\left(E_{x}\right)^{*}
$$

for all $x \in M$; if $F \rightarrow M$ is another vector bundle, we can define the bundle $\operatorname{Hom}(E, F) \rightarrow M$ with fibres

$$
\operatorname{Hom}(E, F)_{x}=\operatorname{Hom}\left(E_{x}, F_{x}\right) ;
$$

similarly, since we will always be working over vector bundles with finite rank, we can define the tensor product as

$$
(E \otimes F)_{x}=\operatorname{Hom}\left(E_{x}^{*}, F_{x}\right) .
$$

Moreover, it is possible to take pullbacks of vector bundles. Given a smooth map $f: M \rightarrow N$ and a vector bundle $E \rightarrow N$, we can form the pullback bundle $f^{*} E \rightarrow M$ by letting

$$
\left(f^{*} E\right)_{x}=E_{f(x)} .
$$

Example 1.2. A classical example of a vector bundle is the tangent bundle $T M \rightarrow M$, and its sections are vector fields, $\Gamma(T M)=\mathcal{X}(M)$. A manifold whose tangent bundle is trivializable is called parallelizable. More interestingly, differential forms are sections of a vector bundle, $\Omega^{k}(M)=\Gamma\left(\Lambda^{k} T^{*} M\right)$.

Definition 1.3. A connection on a vector bundle $E \rightarrow M$ is a bilinear map

$$
\begin{aligned}
\nabla: \mathcal{X} \times \Gamma(E) & \longrightarrow \Gamma(E) \\
(X, s) & \mapsto \nabla_{X}(s)
\end{aligned}
$$

satisfying

- $\nabla_{f X} s=f \nabla_{X} s$,

- $\nabla_{X}(f s)=f \nabla_{X} s+X(f) s$ (Leibniz rule)

\footnotetext{
${ }^{1}$ One says that morphisms like this "cover the identity", because it is also possible to define morphism between vector bundles over different bases, say $E \rightarrow M$ and $F \rightarrow N$. In this case a map $f: M \rightarrow N$ is also needed, and then $u_{x}: E_{x} \rightarrow F_{f(x)}$.
} 
for all $X \in \mathcal{X}(M), s \in \Gamma(E)$ and $f \in C^{\infty}(M)$.

On a given trivialization of the vector bundle with frame $e=\left(e_{1}, \ldots, e_{r}\right)$, the connection is uniquely characterized by a connection matrix $A:=\left(A_{i j}\right)$, which is an $r$-by- $r$ matrix of 1-forms, $A_{i j} \in \Omega^{1}(M)$,

$$
\nabla_{X}^{A}\left(e_{j}\right)=\sum_{i=1}^{r} A_{i j}(X) e_{i}
$$

and we denote by $\nabla^{A}$ the connection associated to the connection matrix $A$. Using the Leibniz rule, on a local section

$$
s=\sum_{i=1}^{r} f^{i} e_{i}
$$

we have

$$
\nabla_{X}^{A} s(x)=\sum_{i} \mathrm{~d} f^{i}\left(X_{x}\right) e_{i}(x)+\sum_{i, j} f^{j}(x) A_{i j}\left(X_{x}\right) e_{i}(x) .
$$

Another way to interpret a connection is as a covariant derivative,

$$
\mathrm{d}_{A}: \Gamma(E) \longrightarrow \Omega^{1}(M, E), \mathrm{d}_{A}(s)(X):=\nabla_{X}^{A}(s) .
$$

From the properties of the connection it is immediate that the covariant derivative is linear and satisfies the Leibniz rule

$$
\mathrm{d}_{A}(f s)=f \mathrm{~d}_{A} s+\mathrm{d} f \otimes s .
$$

Moreover, from (1.1) we see that on a local section $s$ defined in terms of a local frame $e$ as above,

$$
\mathrm{d}_{A} s=\sum_{i} \mathrm{~d} f^{i} e_{i}+\sum_{i, j} f^{j} A_{i j} e_{i},
$$

which leads to the frequently used notation

$$
\mathrm{d}_{A}=\mathrm{d}+A
$$

for the local representation of the covariant derivative.

There are two usual ways to extend the covariant derivative from sections to more general $k$-forms on the vector bundle, and we define two operators

$$
\nabla^{A}, \mathrm{~d}_{A}: \Omega^{k}(M, E) \rightarrow \Omega^{k+1}(M, E)
$$

as follows: for $X_{0}, \ldots, X_{k} \in T M$ and $\omega \in \Omega^{k}(M, E)$,

$$
\begin{array}{r}
\nabla^{A} \omega\left(X_{0}, \ldots, X_{k}\right):=\nabla_{X_{0}}^{A}\left(\omega\left(X_{1}, \ldots, X_{k}\right)\right)-\omega\left(\nabla_{X_{0}}^{M} X_{1}, \ldots, X_{k}\right) \\
-\ldots-\omega\left(X_{1}, \ldots, \nabla_{X_{0}}^{M} X_{k}\right),
\end{array}
$$

and

$$
\begin{aligned}
\mathrm{d}_{A} \omega\left(X_{0}, \ldots, X_{k}\right) & :=\sum_{i=0}^{k}(-1)^{i} \nabla_{X_{i}}^{A}\left(\omega\left(X_{0}, \ldots, \hat{X}_{i}, \ldots, X_{k}\right)\right) \\
& +\sum_{i<j}(-1)^{i+j} \omega\left(\left[X_{i}, X_{j}\right], X_{0}, \ldots, \hat{X}_{i}, \ldots, \hat{X}_{j}, \ldots, X_{k}\right),
\end{aligned}
$$


where $\nabla^{M}$ is the Levi-Civita connection on $T M \rightarrow M$. 2 2 These operators are related to one another by

$$
\mathrm{d}_{A} \omega\left(X_{0}, \ldots, X_{k}\right)=\sum_{i=0}^{k}(-1)^{i} \nabla^{A} \omega\left(X_{i}, X_{0}, \ldots, \hat{X}_{i}, \ldots, X_{k}\right),
$$

and as expected when $k=0$ (that is, in sections of the bundle), $\mathrm{d}_{A}=\nabla^{A}$, so that the notation $\mathrm{d}_{A} s(X)=\nabla_{X}^{A} s$ is still consistent.

Just as we can define new bundles from old using operations which are natural to vector spaces and pullbacks, the same can be done with connections. Let $E \rightarrow M$ be a vector bundle, and let $f: N \rightarrow M$ be a smooth map. Given a connection $\nabla$ on $E$ we can define a pullback connection $f^{*} \nabla$ on $f^{*} E$ as follows: for $s \in \Gamma(E), x \in N$ and $X \in T N$,

$$
\left(f^{*} \nabla\right)_{X}\left(f^{*} s\right)(x)=\nabla_{\mathrm{d}_{x} f(X)} s(f(x)) .
$$

This can be used to define a further useful property of a connection: it defines a way to move from one fibre $E_{x}$ to another along paths on $M$. Given a path on the base, say $\gamma: I \rightarrow M$ for some interval $I \subseteq \mathbb{R}$, we can define paths on $E$ above $\gamma$ as a section $u \in \Gamma\left(\gamma^{*} E\right)$, and then

$$
\begin{aligned}
& u: I \longrightarrow E, \\
& \quad u(t) \in E_{\gamma(t)} .
\end{aligned}
$$

Such a path $u$ is said to be parallel to $\gamma$ if

$$
\left(\gamma^{*} \nabla\right)_{\frac{\mathrm{d}}{\mathrm{d} t}} u=0 .
$$

Given $\gamma, \nabla$ and an initial point $s \in E_{\gamma\left(t_{0}\right)}$ for $t_{0} \in I$, there is a unique path $u_{s}$ such that $u_{s}\left(t_{0}\right)=s$ and $u_{s}$ is parallel to $\gamma$. A collection of such paths taking each point in $E_{\gamma\left(t_{0}\right)}$ to $E_{\gamma\left(t_{1}\right)}$ for $I=\left[t_{0}, t_{1}\right]$ is called parallel transport,

$$
\begin{aligned}
P_{\gamma}^{t_{1}, t_{0}}: E_{\gamma\left(t_{0}\right)} & \rightarrow E_{\gamma\left(t_{1}\right)} \\
s & \mapsto u_{s}\left(t_{1}\right),
\end{aligned}
$$

and this is a linear isomorphism of the fibres it connects.

One last thing that needs to be discussed is vector bundle metric. A metric on a vector bundle $E \rightarrow M$ is a family

$$
h=\left\{h_{x}\right\}_{x \in M}
$$

of inner products on the vector spaces $E_{x}$, which vary smoothly on $M$ in the sense that, for two sections $s, s^{\prime} \in \Gamma(E)$,

$$
h\left(s, s^{\prime}\right)(x)=h_{x}\left(s(x), s^{\prime}(x)\right)
$$

is smooth as a function $M \rightarrow \mathbb{K}$. Given a vector bundle $E \rightarrow M$ with metric $h$, a connection on $E$ is said to be compatible with $h$ if, for instance,

$$
X h\left(s, s^{\prime}\right)=h\left(\nabla_{X} s, s^{\prime}\right)+h\left(s, \nabla_{X} s^{\prime}\right)
$$

holds for every $X \in \mathcal{X}(M)$ and $s, s^{\prime} \in \Gamma(E)$.

Proposition 1.4 ([Cra15], proposition 1.41). Every vector bundle admits a metric, and for every metric there always exists a compatible connection.

\footnotetext{
${ }^{2}$ See the discussion below on Riemannian manifolds for the definition of the Levi-Civita connection.
} 


\subsubsection{Useful notions on Riemannian manifolds}

Before moving on from vector bundles, let us discuss a bit about the very important and special case of the tangent bundle. A Riemannian metric on a manifold $M$ is simply a metric on the tangent bundle, therefore by the previous proposition we know that every smooth manifold admits a Riemannian metric. We will denote a Riemannian metric by $g$.

When $E=T M$, the sections of the vector bundle are also vector fields, and so a connection is an operator

$$
\nabla: \mathcal{X}(M) \times \mathcal{X}(M) \rightarrow \mathcal{X}(M)
$$

which makes it possibly to talk about torsion,

$$
T_{\nabla}(X, Y)=\nabla_{X} Y-\nabla_{Y} X-[X, Y] .
$$

A connection is said to be torsion-free if $T_{\nabla}=0$. Because of the existence of torsion, in this case it is possible to single out a canonical connection:

Proposition 1.5 (Cra15, theorem 1.43). On a Riemannian manifold there exists a unique connection compatible with the metric and torsion-free. It is called the Levi-Civita connection.

It will be useful to define the Christoffel symbols for the Levi-Civita connection. On $T M \rightarrow M$ it is natural to use a local frame

$$
\frac{\partial}{\partial x_{1}}, \ldots, \frac{\partial}{\partial x_{n}}
$$

induced by a local coordinate chart $\left(U, x_{1}, \ldots, x_{n}\right)$. Let $\partial_{i}:=\frac{\partial}{\partial x_{i}}$ and $\nabla_{i}:=\nabla_{\partial_{i}}$, then the Christoffel symbols $\Gamma_{i j}^{k}$ are defined as ${ }^{3}$

$$
\nabla_{i} \partial_{j}=\Gamma_{i j}^{k} \partial_{k}
$$

The fact that $\nabla$ is torsion-free is reflected in the symmetry of the symbols,

$$
\Gamma_{i j}^{k}=\Gamma_{j i}^{k} .
$$

Moreover, representing the metric $g$ locally by a matrix

$$
g_{i j}:=g\left(\partial_{i}, \partial_{j}\right)
$$

and letting $\left(g^{i j}\right)$ be its inverse, the compatibility of $\nabla$ with the metric becomes

$$
\Gamma_{i j}^{k}=\frac{1}{2} \sum_{l}\left(\frac{\partial g_{j l}}{\partial x_{i}}+\frac{\partial g_{i l}}{\partial x_{j}}-\frac{\partial g_{i j}}{\partial x_{l}}\right) g^{l k}
$$

The Christoffel symbols are also useful to show the dependence of the covariant derivative on the metric on the base manifold. For instance, we can write locally for $A \in \Omega^{1}(M, E)$ :

$$
(\nabla A)_{i j}:=\nabla A\left(\partial_{i}, \partial_{j}\right)=\nabla_{i}^{E}\left(A\left(\partial_{j}\right)\right)-A\left(\nabla_{j}^{M} \partial_{i}\right)=\nabla_{i}^{E} A_{j}-\Gamma_{i j}^{k} A_{k} .
$$

Besides a canonical choice of connection, the metric on $M$ also gives natural definitions to operations such as integration and inner products, and then a further choice of vector

\footnotetext{
${ }^{3}$ We use the Einstein summation convention for repeated indices.
} 
bundle metric extends these notions to bundle valued objects. We give these definitions now.

First, the metric defines a volume form $\mathrm{d} v o l$ and then for $f \in C_{0}(M)$ we can define

$$
\int_{M} f:=\int_{M} f \mathrm{~d} v o l \text {. }
$$

Locally, the volume form is given by $\mathrm{d} v o l=\sqrt{|\operatorname{det} g|} \mathrm{d} x_{1} \wedge \cdots \wedge \mathrm{d} x_{n}$. For simplicity we are assuming that $M$ is oriented in order to have a globally defined volume form, however that is not necessary for defining integration, see e.g. [Aub82, chapter 1, sections 9 and 11.

Moreover, the metric induces a pointwise inner product on tensors $\alpha, \beta \in \otimes^{k} T_{x}^{*} M$, which can be written as

$$
\langle\alpha, \beta\rangle=g^{i_{1} j_{1}} \cdots g^{i_{k} j_{k}} \alpha_{i_{1} \cdots i_{k}} \beta_{j_{1} \cdots j_{k}} .
$$

This is independent of the local coordinates. Note that a Riemannian metric is itself a tensor, $g \in \Gamma\left(\otimes^{2} T^{*} M\right)$.

The previous definitions can now be used to define the Hodge star, $*: \Omega^{k}(M) \rightarrow$ $\Omega^{n-k}(M)$, as the only map that satisfies

$$
\alpha \wedge * \beta=\langle\alpha, \beta\rangle \mathrm{d} v o l, \quad \forall \alpha, \beta \in \Omega^{k}(M) .
$$

We can also define an inner product on the space of $k$-forms: for $\alpha, \beta \in \Omega^{k}(M)$,

$$
\langle\alpha, \beta\rangle:=\int_{M}\langle\alpha, \beta\rangle \mathrm{d} v o l=\int_{M} \alpha \wedge * \beta .
$$

We will sometimes denote $\mathrm{d} v o l=: * 1$. Note that $*$ is its own inverse up to a sign: for $\alpha \in \Omega^{k}(M), *^{2} \alpha=(-1)^{k(n-k)} \alpha$. Occasionally, when the sign itself is not important, we will simply use $*^{2}= \pm 1$.

The Hodge star is also used to define the codifferential,

$$
\begin{aligned}
\mathrm{d}^{*}: \Omega^{k}(M) & \longrightarrow \Omega^{k-1}(M) \\
\alpha & \mapsto-(-1)^{n(k-1)} * \mathrm{~d} * \alpha .
\end{aligned}
$$

The operator $\mathrm{d}^{*}$ is also called the formal adjoint to the exterior derivative because of the following: for $\alpha \in \Omega^{k}(M)$ and $\beta \in \Omega^{k+1}(M)$,

$$
\begin{aligned}
\int_{M} \mathrm{~d}(\alpha \wedge * \beta) & =\int_{M} \mathrm{~d} \alpha \wedge * \beta+(-1)^{k} \alpha \wedge \mathrm{d} * \beta \\
& =\int_{M} \mathrm{~d} \alpha \wedge * \beta+(-1)^{k}(-1)^{(n-k+1)(k-1)} \alpha \wedge(* *) \mathrm{d} * \beta \\
& =\int_{M} \mathrm{~d} \alpha \wedge * \beta+(-1)^{n(k-1)} \alpha \wedge *(* \mathrm{~d} *) \beta \\
& =\int_{M} \mathrm{~d} \alpha \wedge * \beta+-\alpha \wedge * \mathrm{~d}^{*} \beta \\
& =\langle\mathrm{d} \alpha, \beta\rangle-\left\langle\alpha, \mathrm{d}^{*} \beta\right\rangle .
\end{aligned}
$$

Then by Stokes's theorem, if either $M$ has no boundary or one of the forms vanishes on $\partial M$

$$
\langle\mathrm{d} \alpha, \beta\rangle=\left\langle\alpha, \mathrm{d}^{*} \beta\right\rangle .
$$

Finally, it is possible to extend these notions to vector bundle valued differential forms. Let $E \rightarrow M$ be such a vector bundle, with bundle metric $h$. Given a local frame $e$ on 
a trivializing neighbourhood $U \subseteq M$, for $\alpha \in \Omega^{k}(M, E)$ and $\beta \in \Omega^{l}(M, E)$ we can write $\alpha=\alpha^{i} \otimes e_{i}$ and $\beta=\beta^{i} \otimes e_{i}$, for $\alpha^{i}, \beta^{i} \in \Omega^{\bullet}(M)$. Then $h$ induces a pairing

$$
\langle\cdot \wedge \cdot\rangle: \Omega^{k}(M, E) \times \Omega^{l}(M, E) \rightarrow \Omega^{k+l}(M),
$$

which is given locally by

$$
\langle\alpha \wedge \beta\rangle:=\left(\alpha^{i} \wedge \beta^{j}\right) h\left(e_{i}, e_{j}\right) .
$$

There is also a natural extension for the Hodge star, given locally by

$$
* \alpha:=\left(* \alpha^{i}\right) \otimes e_{i} .
$$

Note that now for any $\alpha, \beta \in \Omega^{k}(M, E)$ we can associate a top-form on $M$ given by $\langle\alpha \wedge * \beta\rangle$, and thus we define an inner product on $\Omega^{k}(M, E)$,

$$
\langle\alpha, \beta\rangle=\int_{M}\langle\alpha \wedge * \beta\rangle
$$

For the purposes of later use, we will actually refer to

$$
\langle\alpha, \beta\rangle:=*\langle\alpha \wedge * \beta\rangle \in \Omega^{n}(M)
$$

as the (pointwise) inner product on $\Omega^{k}(M, E)$.

Moreover, if $A$ is a connection on the vector bundle $E \rightarrow M$, we may also define the formal adjoint of the covariant derivative $\mathrm{d}_{A}$,

$$
\begin{gathered}
\mathrm{d}_{A}^{*}: \Omega^{k}(M, E) \rightarrow \Omega^{k-1}(M, E), \\
\mathrm{d}_{A}^{*}=-(-1)^{n(k-1)} * \mathrm{~d}_{A} * .
\end{gathered}
$$

\section{$1.2 \quad$ Principal bundles}

Principal bundles are the underlying objects in gauge theory. Our main objects of study, connections and gauge transformations, will be defined in this context, and in fact will be defined in more than one equivalent way. There is much more that can be said about principal bundles and their relationship to vector bundles 4 but our focus in this section will be on the many ways the bundle, connections and gauge transformations can be defined. For organizational purposes, and because we will refer back to them often, all of the local results are collected in a separate subsection.

Definition 1.6. A principal $G$-bundle is a manifold $P$ along with

- a $G$ action on $P, P \times G \rightarrow P,(p, g) \mapsto p g$,

- a surjective map $\pi: P \rightarrow M$ that is $G$-invariant, i.e. $\pi(p g)=\pi(p)$ for all $p \in P$ and $g \in G$,

and such that a local triviality condition is satisfied: for all $x \in M$, there exists a neighbourhood $U$ and a diffeomorphism

$$
\begin{aligned}
\Phi: \pi^{-1}(U) & \longrightarrow U \times G \\
p & \mapsto(\pi(p), \phi(p))
\end{aligned}
$$

taking a fibre $\pi^{-1}(x)$ to $\{x\} \times G$ and which is $G$-equivariant, that is $\phi(p g)=\phi(p) g$.

\footnotetext{
${ }^{4}$ Frame bundles and $G$-structures, for instance. See Cra15.
} 
Equivalently, $\pi: P \rightarrow M$ is a principal $G$-bundle if $\pi$ is a submersion and there is a free and proper $G$-action on $P$ which is fibre preserving. The action is also transitive on the fibres, that is, for any $p, q \in \pi^{-1}(x)$ there exists $g \in G$ such that $q=p g$. Unlike vector bundles, principal bundles do not generally have global sections; indeed, a global section is equivalent to a trivialization of the bundle, $P \simeq M \times G$.

A more direct point of view is to use a bundle atlas $\left(U_{\alpha}, \Phi_{\alpha}=\left(\pi, \phi_{\alpha}\right)\right)_{\alpha \in A}$ to write any $p \in P$ as

$$
p=\lfloor\alpha, x, g\rceil
$$

for $\alpha \in A, x=\pi(p)$ and $g=\phi_{\alpha}(p) \in G$. This is an equivalence class, and we will want that on non-empty intersections $U_{\alpha} \cap U_{\beta}$

$$
\left\lfloor\alpha, x, \phi_{\alpha}(p)\right\rceil=\left\lfloor\beta, x, \phi_{\beta}(p)\right\rceil .
$$

We define so called transition functions $\phi_{\alpha \beta}: U_{\alpha} \cap U_{\beta} \rightarrow G$ as

$$
\phi_{\alpha \beta}(x)=\phi_{\alpha}(p) \phi_{\beta}(p)^{-1}
$$

for any $p \in \pi^{-1}(x)$; this is well defined, as for any $q \in \pi^{-1}(x)$ there exists $g \in G$ such that $q=p g$ and

$$
\phi_{\alpha}(p g) \phi_{\beta}^{-1}(p g)=\phi_{\alpha}(p) g\left(\phi_{\beta}(p) g\right)^{-1}=\phi_{\alpha}(p) \phi_{\beta}(p)^{-1} .
$$

Then the equivalence relation that defines $\lfloor\cdot, \cdot, \cdot\rceil$ will be

$$
(\alpha, x, g) \sim(\beta, x, h) \Longleftrightarrow h=\phi_{\beta \alpha}(x) g .
$$

The $G$-action is defined naturally as

$$
p g=\left\lfloor\alpha, \pi(p), \phi_{\alpha}(p) g\right\rceil .
$$

Note that these functions satisfy cocycle conditions, on $U_{\alpha} \cap U_{\beta} \cap U_{\gamma}$

$$
\phi_{\alpha \gamma}(x)=\phi_{\alpha \beta}(x) \phi_{\beta \gamma}(x)
$$

and also $\phi_{\alpha \alpha}=\mathbb{1}$ and $\phi_{\alpha \beta}(x)^{-1}=\phi_{\beta \alpha}(x)$.

The open cover of $M$ and the transition functions defined on the intersections encode the whole principal bundle. Indeed, given such a cover $M=\bigcup_{\alpha \in A} U_{\alpha}$ and $G$-valued transition functions $\left\{\phi_{\alpha \beta}\right\}_{\alpha, \beta \in A}$, the equivalence relation $(\alpha, x, g) \sim\left(\beta, x, \phi_{\beta \alpha}(x) g\right)$ gives rise to a principal $G$-bundle

$$
\pi:\{\lfloor\alpha, x, g\rceil: \alpha \in A, x \in M, g \in G\} \rightarrow M .
$$

If these transition functions originated from a pre-existing bundle $P$, then this procedure reconstructs $P$. More generally, it is possible to check if two principal $G$-bundles are isomorphic by observing their transition functions on the same open cover of the base bundle. A $G$-bundle isomorphism is a bundle isomorphism 5 that also preserves the group action.

Lemma 1.7. Let $M=\bigcup_{\alpha \in A} U_{\alpha}$ be an open cover of $M$ and let $\left\{\phi_{\alpha \beta}\right\},\left\{\psi_{\alpha \beta}\right\}, \alpha, \beta \in A$ be two sets of transition functions for two principal $G$-bundles over $M$. Then these bundles are isomorphic if, and only if, there exist a cover $M=\bigcup_{\alpha \in A} V_{\alpha}$ with $V_{\alpha} \subseteq U_{\alpha}$ and local functions $g_{\alpha}: V_{\alpha} \rightarrow G$ such that $\psi_{\alpha \beta}(x)=g_{\alpha}(x) \phi_{\alpha \beta}(x) g_{\beta}^{-1}(x)$.

\footnotetext{
${ }^{5} \mathrm{~A}$ diffeomorphism of the total spaces which preserves the fibres.
} 
Isomorphic bundles will usually be identified, and so an object of great importance is the group of $G$-bundle automorphisms of a principal bundle $P$,

$$
\operatorname{Aut}(P):=\{\psi: P \rightarrow P: \pi \circ \psi=\psi \text { and } \psi(p g)=\psi(p) g\} .
$$

To each $\psi \in \operatorname{Aut}(P)$ we will associate a map called a gauge transformation. To properly discuss these transformations and connections on principal bundles, we need to look at associated bundles.

Let $F$ be some other manifold with a representation $\rho: G \rightarrow \operatorname{Diff}(F)$ which gives a $G$-action on it. Then we may define an associated bundle to a principal $G$-bundle $P$ as the set of equivalence classes $\lfloor p, f\rceil=\left\lfloor p g, \rho\left(g^{-1}\right) f\right\rceil$ for all $g \in G$, and we denote it $E(P, F)=(P \times F) / G$. If $F=V$ is a vector space, then $\rho: G \rightarrow G L(V)$ is a representation of $G$ in the usual sense, and $E(P, V)$ is a vector bundle. We will now define and give properties of two bundles associated to $P$ which will be especially important.

We denote by $\tilde{\pi}: \operatorname{Ad}(P) \rightarrow M$ the associated bundle with fibre $G$

$$
\operatorname{Ad}(P):=\frac{P \times G}{G}
$$

where $G$ acts on itself by conjugation, $(g, h) \mapsto h g h^{-1}$. Thus we have the fibres

$$
\tilde{\pi}^{-1}(x)=\operatorname{Ad}(P)_{x}=\{\lfloor p, g\rceil: g \in G\},
$$

for $p \in \pi^{-1}(x)$, and note that $\lfloor p g, h\rceil=\left\lfloor p, g h g^{-1}\right\rceil$. We can identify the sections of $\operatorname{Ad}(P)$ with the $G$-equivariant functions,

$$
C^{\infty}(P, G)^{G}:=\left\{u: P \rightarrow G: u(p g)=g^{-1} u(p) g=\operatorname{Ad}_{g^{-1}} u(p)\right\},
$$

in the following way:

- a section $s \in \Gamma \operatorname{Ad}(P)$ induces a function $u$ by $s(\pi(p))=\lfloor p, u(p)\rceil$, and $u$ is equivariant because

$$
\lfloor p, u(p)\rceil=\lfloor p g, u(p g)\rceil=\left\lfloor p, \operatorname{Ad}_{g} u(p g)\right\rceil
$$

implies $u(p)=g u(p g) g^{-1}$;

- a function $u \in C^{\infty}(P, G)^{G}$ induces a section $s(x)=\lfloor p, u(p)\rceil$, and this does not depend on the choice of $p \in \pi^{-1}(x)$, as for any other $q \in \pi^{-1}(x)$ we write $q=p g$ for some $g \in G$, and so

$$
\lfloor q, u(q)\rceil=\lfloor p g, u(p g)\rceil=\left\lfloor p g, \operatorname{Ad}_{g^{-1}} u(p)\right\rceil=\lfloor p, u(p)\rceil .
$$

Furthermore, the sections of $\operatorname{Ad}(P)$ form a group under pointwise multiplication,

$$
\left(s \cdot s^{\prime}\right)(x):=\left\lfloor p, u(p) u^{\prime}(p)\right\rceil .
$$

The $G$-equivariant functions can further be identified with the $G$-bundle automorphisms of $P$. This identification can be written as follows:

- A function $u \in C^{\infty}(P, G)^{G}$ induces an automorphism $\psi: P \rightarrow P, \psi(p)=p u(p)$ which is clearly fibre preserving, and is equivariant:

$$
\psi(p g)=p g u(p g)=p u(p) g=\psi(p) g .
$$


- Conversely, there is a unique map $u: P \rightarrow G$ such that $\psi(p)=p u(p)$ for a given $\psi \in \operatorname{Aut}(P)$. The equivariance of $\psi$ gives

$$
\psi(p g)=p(g u(p g))=p(u(p) g)=\psi(p) g,
$$

and because the action of $G$ is free, $g u(p g)=u(p) g$, which establishes $u \in C^{\infty}(P, G)^{G}$.

This identification also introduces a group multiplication in $\operatorname{Aut}(P)$ via composition of automorphisms,

$$
\psi_{1} \circ \psi_{2}(p)=p \cdot u_{2}(p) u_{1}(p) .
$$

Thus we have the group isomorphisms

$$
\Gamma \operatorname{Ad}(P) \simeq C^{\infty}(P, G)^{G} \simeq \operatorname{Aut}(P) .
$$

Definition 1.8 (Gauge transformation). We will call $u \in C^{\infty}(P, G)^{G}$ a gauge transformation, and denote the group of gauge transformations $\mathcal{G}(P)$.

We will have more to say about gauge transformations, their action and local representation, further into the chapter.

Let $\mathfrak{g}$ be the Lie algebra of $G$, and let $G$ act on $\mathfrak{g}$ via the adjoint action

$$
\begin{aligned}
\operatorname{Ad}: G & \rightarrow \operatorname{End}(\mathfrak{g}) \\
\xi & \mapsto \operatorname{Ad}_{g}(\xi)=g \xi g^{-1} .
\end{aligned}
$$

Then $\operatorname{ad}(P) \rightarrow M$ is the associated vector bundle with standard fibre $\mathfrak{g}$,

$$
\operatorname{ad}(P):=\frac{P \times \mathfrak{g}}{G} .
$$

Just as with $\operatorname{Ad}(P)$, we can identify the sections of $\operatorname{ad}(P)$ with $G$-equivariant functions $P \rightarrow \mathfrak{g}$,

$$
C^{\infty}(P, \mathfrak{g})^{G}:=\left\{f: P \rightarrow \mathfrak{g}: f(p g)=\operatorname{Ad}_{g^{-1}} f(p)\right\},
$$

such that any section can be written $s(x)=\lfloor p, f(p)\rceil$, for any $p \in \pi^{-1}(x)$. The space of sections $\Gamma \operatorname{ad}(P)$ has a natural Lie algebra structure induced by the bracket on $\mathfrak{g}$,

$$
\left[s, s^{\prime}\right](x)=\left\lfloor p,\left[f(p), f^{\prime}(p)\right]\right\rceil .
$$

The importance of this associated bundle will become clear after the next definition.

There is a canonical vertical subbundle $T^{V} P \subseteq T P$ given by $T^{V} P=\operatorname{ker} \mathrm{d} \pi$, composed of vectors tangent to the fibres $P_{x} \simeq G$. Each vertical tangent space is isomorphic to the Lie algebra,

$$
T_{p}^{V} P=\operatorname{ker} \mathrm{d}_{p} \pi \simeq \mathfrak{g},
$$

and the tangent vectors will be denoted $p \xi$, as defined in subsection 1.2.2, Every complement of these vertical spaces is isomorphic to $\operatorname{Im}\left(\mathrm{d}_{p} \pi\right)=T_{\pi(p)} M$, but there is in general no canonical choice for these horizontal spaces; a connection on a principal bundle represents precisely a choice of equivariant horizontal distribution $H$ such that $T P=T^{V} P \oplus H$.

Definition 1.9 (Connection). A connection on $P$ is a 1 -form $A \in \Omega^{1}(P, \mathfrak{g})$ satisfying 
- $A_{p g}(v g)=g^{-1} A_{p}(v) g=\operatorname{Ad}_{g^{-1}} A$, for all $g \in G$ and $v \in T_{p} P$,

- $A_{p}(p \xi)=\xi$, for all $\xi \in \mathfrak{g}$,

that is, it is $G$-equivariant and takes fixed values on vertical tangent vectors. We denote the set of smooth connections $\mathcal{A}(P)$.

To see the relation between both notions, observe that we can write

$$
T P=T^{V} P \oplus \operatorname{ker} A .
$$

Note that $\Omega^{1}(P, \mathfrak{g})$ means that the one-forms take values in $\mathfrak{g}$, which is a finite dimensional vector space. In the language of vector bundles, $\Omega^{1}(P, \mathfrak{g})=\Gamma\left(\Lambda T^{*} P \otimes P \times \mathfrak{g}\right)$, where $P \times \mathfrak{g} \rightarrow P$ is a trivializable vector bundle of rank dim $\mathfrak{g}$. From either point of view it is clear that many global results on forms will readily generalize to connections; for instance, it makes sense to write $\mathrm{d} A$ in this case, when in general connections are needed to define covariant derivatives.

It is easy to see from the definition that the difference of two connections is a basic form: $G$-equivariant and horizontal ${ }^{6}$. On the other hand, it is known that the space of basic $k$-forms $\Omega_{\text {bas }}^{k}(P, \mathfrak{g})$ is isomorphic to $\Omega^{k}(M, \operatorname{ad}(P))$ : for $\tau \in \Omega_{b a s}^{k}(P, \mathfrak{g})$, the corresponding $\tilde{\tau} \in \Omega^{k}(M, \operatorname{ad}(P))$ is uniquely defined by

$$
\left\lfloor p, \tau_{p}\left(X_{1}, \ldots, X_{k}\right)\right\rceil=\tilde{\tau}_{\pi(p)}\left(\mathrm{d}_{p} \pi\left(X_{1}\right), \ldots, \mathrm{d}_{p} \pi\left(X_{k}\right)\right) \in \operatorname{ad}(P)_{\pi(p)}
$$

for any $X_{1}, \ldots, X_{k} \in T_{p} P$. Thus, the space of connections is an affine space, and fixing a reference connection $\tilde{A} \in \mathcal{A}(P)$ we can write

$$
\mathcal{A}(P)=\tilde{A}+\Omega^{1}(M, \operatorname{ad}(P)) .
$$

A similar isomorphism exists for general associated vector bundles $E(P, V)$, and it allows a connection on the principal bundle to induce one on the associated bundle. Suppose $\rho: G \rightarrow G L(V)$ is a representation and $E(P, V)$ is the corresponding bundle. There is an isomorphism $h: \Omega^{k}(M, E(P, V)) \stackrel{\sim}{\longrightarrow} \Omega_{b a s}^{k}(P, V)$, and furthermore we can differentiate the representation, $\mathrm{d}_{\mathbb{1}} \rho: \mathfrak{g} \rightarrow \operatorname{End}(V)$. For $A \in \mathcal{A}(P)$ we can then define

$$
\begin{aligned}
\mathrm{d}_{A}: \Omega_{b a s}^{k}(P, V) & \longrightarrow \Omega_{b a s}^{k+1}(P, V) \\
\omega & \mapsto \mathrm{d} f+\mathrm{d}_{\mathbb{1}} \rho(A)(\omega),
\end{aligned}
$$

and this in turn will induce a connection on $E(P, V)$,

$$
\nabla^{A}:=h^{-1} \circ \mathrm{d}_{A} \circ h: \Omega^{k}(M, E(P, V)) \rightarrow \Omega^{k+1}(M, E(P, V)) .
$$

In the particular case of $\operatorname{ad}(P)$, which is the associated vector bundle $E(P, \mathfrak{g})$ with $\rho=\operatorname{Ad}: G \rightarrow G L(\mathfrak{g})$, we have $\mathrm{d}_{\mathbb{1}} \rho(\xi) \eta=\operatorname{ad}(\xi) \eta=[\xi, \eta]$ and so a connection $A \in \mathcal{A}(P)$ defines

$$
\begin{aligned}
\mathrm{d}_{A}: \Omega_{\text {bas }}^{k}(P, \mathfrak{g}) & \longrightarrow \Omega_{\text {bas }}^{k+1}(P, \mathfrak{g}) \\
\tau & \mapsto \mathrm{d} \tau+[A \wedge \tau],
\end{aligned}
$$

\footnotetext{
${ }^{6}$ It kills vertical tangent vectors.
} 
where $[\cdot \wedge \cdot]$ is the wedge product of two forms with the Lie bracket used to combine the values in $\mathfrak{g} \cdot 7$ This will then induce on $\operatorname{ad}(P)$ a covariant derivative

$$
\nabla^{A}: \Gamma(\operatorname{ad}(P)) \longrightarrow \Omega^{1}(M, \operatorname{ad}(P))
$$

which can be written explicitly in the following way: for $s \in C^{\infty}(P, \mathfrak{g})^{G} \simeq \Gamma(\operatorname{ad}(P))$, $X \in T_{x} M$ and $Y \in T_{p} P$ such that $\pi(p)=x$ and $\mathrm{d}_{p} \pi(Y)=X$,

$$
\nabla^{A} s(X)=\left\lfloor p, \mathrm{~d}_{p} s(Y)+[A(Y), s(p)]\right\rceil \in \operatorname{ad}(P)_{x} .
$$

This, of course, extends to $\nabla^{A}: \Omega^{k}(M, \operatorname{ad}(P)) \rightarrow \Omega^{k+1}(M, \operatorname{ad}(P))$ exactly as in 1.2 .

Now, while it is true that for the usual (de Rham) exterior derivative we have $\mathrm{d}^{2}=0$, this will not hold in general for $\mathrm{d}_{A}$ defined above. This failure can be measured by the curvature of the connection,

$$
F_{A}=\mathrm{d} A+\frac{1}{2}[A \wedge A] \in \Omega_{b a s}^{2}(P, \mathfrak{g}),
$$

and we obtain $\mathrm{d}_{A} \mathrm{~d}_{A} \tau=\left[F_{A} \wedge \tau\right]$ for all $\tau \in \Omega_{b a s}^{k}(P, \mathfrak{g})$. A connection for which $F_{A}=0=\mathrm{d}_{A}^{2}$ is called a flat connection. The curvature satisfies the Bianchi identity,

$$
\mathrm{d}_{A} F_{A}=0 .
$$

Moreover, it can be seen as a differential form in $\Omega^{2}(M, \operatorname{ad}(P))$.

We now calculate the effect of a gauge transformation on a connection and its curvature. For that, define the Maurer-Cartan form $\theta_{g}=\mathrm{d}_{g} L_{g^{-1}}: T_{g} G \rightarrow \mathfrak{g}$, for $L_{g}(h)=g h$.

Lemma 1.10 (The gauge action). Let $u \in \mathcal{G}(P)$ and $\psi(p)=p u(p)$ its corresponding automorphism. Then the action of a gauge transformation on a connection is defined as $u^{*} A:=\psi^{*} A$ and can be written

$$
\begin{aligned}
u^{*} A & =u^{-1} A u+u^{*} \theta \\
& =u^{-1} A u+u^{-1} \mathrm{~d} u .
\end{aligned}
$$

Proof. Define the multiplication $\sigma$ on $P \times G$ as $\sigma(p, g)=p g=\tilde{R}_{g}(p)=\tilde{L}_{p}(g)$ and write $\psi(p)=\sigma(I d, u)(p)$. Then for $v \in T_{p} P$,

$$
\begin{aligned}
\mathrm{d}_{p} \psi(v) & =\mathrm{d}_{(p, u(p))} \sigma \circ \mathrm{d}_{p}(I d, u)(v) \\
& =\mathrm{d}_{(p, u(p))} \sigma\left(v, \mathrm{~d}_{p} u(v)\right) \\
& \stackrel{(*)}{=} \mathrm{d}_{p} \tilde{R}_{u(p)}(v)+\mathrm{d}_{u(p)} \tilde{L}_{p}\left(\mathrm{~d}_{p} u(v)\right),
\end{aligned}
$$

where $(*)$ follows because $T_{(p, g)}(P \times G) \simeq T_{p} P \oplus T_{g} G$ and using curves one can easily show that $\mathrm{d}_{(p, g)} \sigma(v, 0)=\mathrm{d}_{p} \tilde{R}_{g}(v)$ and $\mathrm{d}_{(p, g)} \sigma(0, X)=\mathrm{d}_{g} \tilde{L}_{p}(X)$. Since $\tilde{L}_{p}(h)=p h=p g g^{-1} h$, we may write $\tilde{L}_{p}=\tilde{L}_{p g} \circ L_{g^{-1}}$, so that

$$
\mathrm{d}_{u(p)} \tilde{L}_{p}\left(\mathrm{~d}_{p} u(v)\right)=\mathrm{d}_{\mathbb{I}} \tilde{L}_{p u(p)} \mathrm{d}_{u(p)} L_{u(p)^{-1}}\left(\mathrm{~d}_{p} u(v)\right)=\mathrm{d}_{\mathbb{I}} \tilde{L}_{p u(p)} \theta_{u(p)}\left(\mathrm{d}_{p} u(v)\right)
$$

For $p \xi=\mathrm{d}_{\mathbb{1}} \tilde{L}_{p}(\xi)$ and $v g=\mathrm{d}_{p} \tilde{R}_{g}(v)$ as defined in subsection 1.2 .2

$$
\mathrm{d}_{p} \psi(v)=v u(p)+p u(p) \theta_{u(p)}\left(\mathrm{d}_{p} u(v)\right) .
$$

\footnotetext{
${ }^{7}$ For example, for $\alpha, \beta \in \Omega_{b a s}^{1}(P, \mathfrak{g})$ and $X, Y \in T_{p} P,[\alpha \wedge \beta](X, Y)=[\alpha(X), \beta(Y)]-[\alpha(Y), \beta(X)]$, and then note $[\alpha \wedge \alpha]=2[\alpha, \alpha]$.
} 
Now, using this expression and calculating using the properties of the connection,

$$
\begin{aligned}
\psi^{*} A(v) & =A_{\psi(p)}\left(\mathrm{d}_{p} \psi(v)\right) \\
& \left.=A_{p u(p)}(v u(p)+p u(p) \underbrace{\theta_{u(p)}\left(\mathrm{d}_{p} u(v)\right.}_{\in \mathfrak{g}})\right) \\
& =u(p)^{-1} A_{p}(v) u(p)+\theta_{u(p)}\left(\mathrm{d}_{p} u(v)\right),
\end{aligned}
$$

and rewriting $\theta_{u(p)}\left(\mathrm{d}_{p} u(v)\right)$ as $\left(u^{*} \theta\right)_{p}(v)$ or $\mathrm{d}_{u(p)} L_{u(p)^{-1}}\left(\mathrm{~d}_{p} u(v)\right)=u(p)^{-1} \mathrm{~d}_{p} u(v)$ from $g v_{h}=\mathrm{d}_{h} L_{g}(v)$, we have the expressions we wanted for the gauge action.

From this, it is also possible to show the effect of a gauge transformation on the curvature,

$$
F_{u^{*} A}=u^{*} F_{A}=u^{-1} F_{A} u \text {. }
$$

Finally, we define a pointwise inner product on $\Omega^{k}(M, \operatorname{ad}(P))$ as in (1.7). We assume the Riemannian metric on $M$ is given, and the metric on $\operatorname{ad}(P)$ is induced by the Ad-invariant inner product on $\mathfrak{g}$ defined in subsection 1.2 .2 .

$$
\langle\lfloor p, \xi\rceil,\lfloor p, \zeta\rceil\rangle_{\operatorname{ad}(P)}:=\langle\xi, \zeta\rangle_{\mathfrak{g}}
$$

Now, viewing the curvature as being in $\Omega^{2}(M, \operatorname{ad}(P))$ and writing $|\cdot|=\sqrt{\langle\cdot, \cdot\rangle}: M \rightarrow \mathbb{R}$, we can define the Yang-Mills functional

$$
\mathcal{Y} \mathcal{M}(A):=\int_{M}\left|F_{A}\right|^{2} \mathrm{~d} v o l=\int_{M} *\left\langle F_{A} \wedge * F_{A}\right\rangle
$$

Due to the gauge action on $F_{A}$ and the invariance of the metric on $\operatorname{ad}(P)$, this functional is invariant under gauge transformations,

$$
\mathcal{Y} \mathcal{M}\left(u^{*} A\right)=\mathcal{Y} \mathcal{M}(A), \quad \forall u \in \mathcal{G}(P)
$$

We will want to study its extrema. For that, note that because $\mathcal{A}(P)$ is an affine space with vector space $\Omega^{1}(M, \operatorname{ad}(P))$, it is enough to check the variation of the curvature along lines $A+t \beta$, for $\beta \in \Omega^{1}(M, \operatorname{ad}(P))$. Now,

$$
F_{A+t \beta}=F_{A}+t \mathrm{~d}_{A} \beta+\frac{1}{2} t^{2}[\beta \wedge \beta],
$$

and thus momentarily writing $\langle\cdot, \cdot\rangle$ for the integrated (not pointwise) inner product it is straightforward to calculate

$$
\begin{aligned}
\left.\frac{\mathrm{d}}{\mathrm{d} t}\right|_{t=0} \mathcal{Y} \mathcal{M}(A+t \beta) & =\left.\frac{\mathrm{d}}{\mathrm{d} t}\right|_{t=0}\left\langle F_{A+t \beta}, F_{A+t \beta}\right\rangle \\
& =2\left\langle F_{A}, \mathrm{~d}_{A} \beta\right\rangle,
\end{aligned}
$$

and so an extremum of the $\mathcal{Y} \mathcal{M}$ functional has to satisfy the weak Yang-Mills equation,

$$
\int_{M}\left\langle F_{A}, \mathrm{~d}_{A} \beta\right\rangle=0, \quad \forall \beta \in \Omega^{1}(M, \operatorname{ad}(P)) .
$$


If everything is smooth, this is equivalent to the (strong) Yang-Mills equation,

$$
\begin{cases}\mathrm{d}_{A}^{*} F_{A}=0 & \text { on } M, \\ \left.* F_{A}\right|_{\partial M}=0 & \text { on } \partial M,\end{cases}
$$

where in the case of a manifold without boundary this is just the usual Yang-Mills equation. This will be proved further ahead in a more general context as lemma 1.37. Just as the functional, these equations are also invariant under gauge transformations, and because of this it is said that the solutions to the Yang-Mills equations come in gauge orbits.

\subsubsection{Local formulations and results}

In this subsection we will derive local representations and formulas for the objects we have defined, as these will be the forms which we will mainly use throughout the text.

Recall the description of a principal $G$-bundle $\pi: P \longrightarrow M$ via local trivializations and transition functions given at the beginning of this section. On a trivializing open set $U_{\alpha} \subseteq M$ we have a bundle chart

$$
\begin{aligned}
\Phi_{\alpha}: \pi^{-1}\left(U_{\alpha}\right) & \longrightarrow U_{\alpha} \times G \\
p & \mapsto\left(\pi(p), \phi_{\alpha}(p)\right),
\end{aligned}
$$

and the transition functions defined on intersections $U_{\alpha} \cap U_{\beta}$

$$
\phi_{\alpha \beta}(x)=\phi_{\alpha}(p) \phi_{\beta}(p)^{-1},
$$

which obey the cocycle conditions 1.8 . Moreover, we write the induced trivializations on the associated bundles $\operatorname{Ad}(P)$ and $\operatorname{ad}(P)$ over $U_{\alpha}$ with the same notation, as there is very little chance of confusion in context. For $\tilde{\pi}: \operatorname{Ad}(P) \longrightarrow M$,

$$
\begin{aligned}
\tilde{\Phi}_{\alpha}: \tilde{\pi}^{-1}\left(U_{\alpha}\right) & \longrightarrow U_{\alpha} \times G \\
\lfloor p, g\rceil & \mapsto\left(\pi(p), \tilde{\phi}_{\alpha}(\lfloor p, g\rceil),\right.
\end{aligned}
$$

with $\tilde{\phi}_{\alpha}(\lfloor p, g\rceil)=\phi_{\alpha}(p) g \phi_{\alpha}(p)^{-1}$. And for $\tilde{\pi}: \operatorname{ad}(P) \rightarrow M$,

$$
\begin{aligned}
\tilde{\Phi}_{\alpha}: \tilde{\pi}^{-1}\left(U_{\alpha}\right) & \longrightarrow U_{\alpha} \times \mathfrak{g} \\
\lfloor p, \xi\rceil & \mapsto\left(\pi(p), \tilde{\phi}_{\alpha}(\lfloor p, \xi\rceil),\right.
\end{aligned}
$$

with $\tilde{\phi}_{\alpha}(\lfloor p, \xi\rceil)=\phi_{\alpha}(p) \xi \phi_{\alpha}(p)^{-1}$. Throughout this subsection we will assume this setting.

For a gauge transformation $u \in \mathcal{G}(P)$, let $\tilde{u} \in \Gamma(\operatorname{Ad}(P))$ be the corresponding section, such that $\tilde{u}(x)=\lfloor p, u(p)\rceil$ for $x=\pi(p)$. Then we can use this to define $u$ locally on $U_{\alpha}$,

$$
u_{\alpha}:=\tilde{\phi}_{\alpha} \circ \tilde{u}: U_{\alpha} \rightarrow G,
$$

and this acts on $U_{\alpha} \times G$ by $(x, g) \mapsto\left(x, g u_{\alpha}(x)\right)$. Thus, for any $p \in \pi^{-1}(x)$,

$$
u_{\alpha}(x)=\phi_{\alpha}(p) u(p) \phi_{\alpha}(p)^{-1}
$$

is well defined, and we can recover $u$ on $U_{\alpha}$,

$$
u(p)=\phi_{\alpha}(p)^{-1} u_{\alpha}(x) \phi_{\alpha}(p) .
$$


If we assume this to be valid for all $\alpha \in A$, then using the transition functions on $U_{\alpha} \cap U_{\beta}$ we get the transition identity

$$
u_{\beta}=\phi_{\alpha \beta}^{-1} u_{\alpha} \phi_{\alpha \beta}
$$

Likewise, any collection of $G$-valued functions $\left\{u_{\alpha}\right\}_{\alpha \in A}$ satisfying 1.18 uniquely defines a global gauge transformation in the same way as we used to recover $u(p)$. Equivalently, a global gauge transformation must satisfy

$$
\phi_{\alpha \beta}=u_{\alpha}^{-1} \phi_{\alpha \beta} u_{\beta}
$$

The local description of connections on open sets $U \subseteq M$ is very similar, using the isomorphism $\Omega_{\text {bas }}^{k}(P, \mathfrak{g}) \simeq \Omega^{k}(M, \operatorname{ad}(P))$ and then the second component of the local trivialization for $\operatorname{ad}(P)$. For $\tau \in \Omega_{\text {bas }}^{k}(P, \mathfrak{g})$ let $\tilde{\tau} \in \Omega^{k}(M, \operatorname{ad}(P))$ be the corresponding form given by $(1.9)$. This defines $\tau$ locally,

$$
\tau_{\alpha}:=\tilde{\phi}_{\alpha} \circ \tilde{\tau} \in \Omega^{k}\left(U_{\alpha}, \mathfrak{g}\right),
$$

and similar to the case of the gauge transformation, on an intersection $U_{\alpha} \cap U_{\beta}$ these local forms will satisfy

$$
\tau_{\beta}=\phi_{\alpha \beta}^{-1} \tau_{\alpha} \phi_{\alpha \beta}
$$

and the global form can be reconstructed as

$$
\tau\left(Y_{1}, \ldots, Y_{k}\right)=\phi_{\alpha}(p)^{-1} \tau_{\alpha}\left(\mathrm{d}_{p} \pi\left(Y_{1}\right), \ldots, \mathrm{d}_{p} \pi\left(Y_{k}\right)\right) \phi_{\alpha}(p), \forall Y_{1}, \ldots, Y_{k} \in T_{p} P .
$$

In the case of connections it is necessary to choose a reference connection in order to use this isomorphism, and there is no canonical choice if the bundle is not flat. However, locally on $\pi^{-1}\left(U_{\alpha}\right)$ there is a natural choice, namely $\tilde{A}_{\alpha}:=\phi_{\alpha}^{-1} \mathrm{~d} \phi_{\alpha}$. Then for the local representative $A_{\alpha} \in \Omega^{1}\left(U_{\alpha}, \mathfrak{g}\right)$ of $A \in \mathcal{A}(P)$ we write

$$
A_{\alpha}\left(\mathrm{d}_{p} \pi(Y)\right)=\phi_{\alpha}(p) A(Y) \phi_{\alpha}(p)^{-1}-\mathrm{d}_{p} \phi_{\alpha}(Y) \phi_{\alpha}(p)^{-1}, \forall Y \in T_{p} P .
$$

Assuming that $A$ can be recovered over $U_{\alpha}$ as

$$
A(Y)=\phi_{\alpha}(p)^{-1} A_{\alpha}\left(\mathrm{d}_{p} \pi(Y)\right) \phi_{\alpha}(p)+\phi_{\alpha}(p)^{-1} \mathrm{~d}_{p} \phi_{\alpha}(Y)
$$

we will once again get a transition identity for the local representatives of connections over intersections. This is stated in the following lemma, along with the local formula for the gauge action, which shows that locally a gauge transformation can be thought of as a change of trivialization.

Lemma 1.11. For a connection $A \in \mathcal{A}(P)$, its local representatives $A_{\alpha}, A_{\beta}$ have to meet the following on $U_{\alpha} \cap U_{\beta}$ :

$$
A_{\beta}=\phi_{\alpha \beta}^{-1} A_{\alpha} \phi_{\alpha \beta}+\phi_{\alpha \beta}^{-1} \mathrm{~d} \phi_{\alpha \beta}
$$

Moreover, the local effect of a gauge transformation is

$$
\left(u^{*} A\right)_{\alpha}=u_{\alpha}^{-1} A_{\alpha} u_{\alpha}+u_{\alpha}^{-1} \mathrm{~d} u_{\alpha} .
$$


Proof. Both of the affirmations are checked with straightforward calculations. For the change in trivialization, omitting the evaluations at $p, Y$ and $\mathrm{d}_{p} \pi(Y)$, we calculate

$$
\begin{aligned}
A_{\beta} & =\phi_{\beta} A \phi_{\beta}^{-1}-\mathrm{d} \phi_{\beta} \cdot \phi_{\beta}^{-1} \\
& =\phi_{\beta}\left(\phi_{\alpha}^{-1} A_{\alpha} \phi_{\alpha}+\phi_{\alpha}^{-1} \mathrm{~d} \phi_{\alpha}\right) \phi_{\beta}^{-1}-\mathrm{d} \phi_{\beta} \cdot \phi_{\beta}^{-1} \\
& =\phi_{\alpha \beta}^{-1} A_{\alpha} \phi_{\alpha \beta}+\phi_{\alpha \beta}^{-1} \mathrm{~d} \phi_{\alpha} \cdot \phi_{\beta}^{-1}-\mathrm{d} \phi_{\beta} \cdot \phi_{\beta}^{-1} \\
& =\phi_{\alpha \beta}^{-1} A_{\alpha} \phi_{\alpha \beta}+\phi_{\alpha \beta}^{-1}\left(\mathrm{~d} \phi_{\alpha} \cdot \phi_{\beta}^{-1}-\phi_{\alpha \beta} \mathrm{d} \phi_{\beta} \cdot \phi_{\beta}^{-1}\right) \\
& =\phi_{\alpha \beta}^{-1} A_{\alpha} \phi_{\alpha \beta}+\phi_{\alpha \beta}^{-1}\left(\mathrm{~d} \phi_{\alpha} \cdot \phi_{\beta}^{-1}+\phi_{\alpha} \phi_{\beta}^{-1} \phi_{\beta} \mathrm{d} \phi_{\beta}^{-1}\right) \\
& =\phi_{\alpha \beta}^{-1} A_{\alpha} \phi_{\alpha \beta}+\phi_{\alpha \beta}^{-1} \mathrm{~d} \phi_{\alpha \beta} .
\end{aligned}
$$

Then for the local gauge action

$$
\begin{aligned}
\left(u^{*} A\right)_{\alpha}= & \phi_{\alpha}\left(u^{*} A\right) \phi_{\alpha}^{-1}-\mathrm{d} \phi_{\alpha} \cdot \phi_{\alpha}^{-1} \\
= & \phi_{\alpha}\left(u^{-1} A u+u^{-1} \mathrm{~d} u\right) \phi_{\alpha}^{-1}-\mathrm{d} \phi_{\alpha} \cdot \phi_{\alpha}^{-1} \\
= & \phi_{\alpha}\left(\phi_{\alpha}^{-1} u_{\alpha}^{-1} \phi_{\alpha} A \phi_{\alpha}^{-1} u_{\alpha} \phi_{\alpha}+\phi_{\alpha}^{-1} u_{\alpha}^{-1} \phi_{\alpha} \mathrm{d}\left(\phi_{\alpha}^{-1} u_{\alpha} \phi_{\alpha}\right)\right) \phi_{\alpha}^{-1}-\mathrm{d} \phi_{\alpha} \cdot \phi_{\alpha}^{-1} \\
= & u_{\alpha}^{-1}\left(\phi_{\alpha} A \phi_{\alpha}^{-1}\right) u_{\alpha} \\
& \quad+u_{\alpha}^{-1} \phi_{\alpha} \mathrm{d} \phi_{\alpha}^{-1} \cdot u_{\alpha}+u_{\alpha}^{-1} \mathrm{~d} u_{\alpha}+\mathrm{d} \phi_{\alpha} \cdot \phi_{\alpha}^{-1} \\
& \quad-\mathrm{d} \phi_{\alpha} \cdot \phi_{\alpha}^{-1} \\
= & u_{\alpha}^{-1} A_{\alpha} u_{\alpha}+u_{\alpha}^{-1} \mathrm{~d} \phi_{\alpha} \cdot \phi_{\alpha}^{-1} u_{\alpha}+u_{\alpha}^{-1} \phi_{\alpha} \mathrm{d} \phi_{\alpha}^{-1} \cdot u_{\alpha}+u_{\alpha}^{-1} \mathrm{~d} u_{\alpha} \\
= & u_{\alpha}^{-1} A_{\alpha} u_{\alpha}+u_{\alpha}^{-1} \mathrm{~d} u_{\alpha} .
\end{aligned}
$$

Finally, the covariant exterior derivative $\mathrm{d}_{A}: \Omega_{\text {bas }}^{k}(P, \mathfrak{g}) \rightarrow \Omega_{\text {bas }}^{k+1}(P, \mathfrak{g})$ induced by a connection $A \in \mathcal{A}(P)$ as in 1.10 can be written locally on $U_{\alpha}$, taking the local representatives $A_{\alpha} \in \Omega^{1}\left(U_{\alpha}, \mathfrak{g}\right)$ and $\tau_{\alpha} \in \Omega^{k}\left(U_{\alpha}, \mathfrak{g}\right)$,

$$
\left(\mathrm{d}_{A} \tau\right)_{\alpha}=\mathrm{d} \tau_{\alpha}+\left[A_{\alpha} \wedge \tau_{\alpha}\right]
$$

its formal adjoint $\mathrm{d}_{A}^{*}: \Omega_{b a s}^{k}(P, \mathfrak{g}) \rightarrow \Omega_{\text {bas }}^{k+1}(P, \mathfrak{g})$ is given locally as

$$
\left(\mathrm{d}_{A}^{*} \tau\right)_{\alpha}=\mathrm{d}^{*} \tau_{\alpha}-(-1)^{(n-k)(k-1)} *\left[A_{\alpha} \wedge * \tau_{\alpha}\right] ;
$$

and the curvature 1.12 can be written

$$
\left(F_{A}\right)_{\alpha}=\mathrm{d} A_{\alpha}+\frac{1}{2}\left[A_{\alpha} \wedge A_{\alpha}\right] \in \Omega^{2}\left(U_{\alpha}, \mathfrak{g}\right),
$$

and transforms under a change of trivialization in the same way it does under a gauge transformation, $\left(F_{A}\right)_{\beta}=\phi_{\alpha \beta}^{-1}\left(F_{A}\right)_{\alpha} \phi_{\alpha \beta}$.

A word on notation. Occasionally, when working locally but not specifying a bundle atlas, we will drop subscripts $\alpha$ and a connection $A \in \mathcal{A}(P)$ will be represented by $A \in$ $\Omega^{1}(U, \mathfrak{g})$, or a gauge transformation $u \in \mathcal{G}(P)$ will be represented by $u: U \rightarrow G$. When specifying a global reference connection $\tilde{A}$, a connection $A \in \mathcal{A}(P)$ will be denoted $A=$ $\tilde{A}+\alpha$, and $\alpha \in \Omega_{\text {bas }}^{1}(P, \mathfrak{g})$ will be its representative. 


\subsubsection{Several results on $G, \mathfrak{g}$ and the action on $P$}

In this subsection we fix some notation pertaining to the action of $G$ on $P$ and $\mathfrak{g}$, define the inner product that will be fixed on $\mathfrak{g}$ and some other notions that depend on it, such as the metric on $G$, and prove a result on the derivative of the adjoint action which will be needed later.

We begin with some notation: For $p \in P$ and $g, h \in G$,

$$
\begin{aligned}
& g h=L_{g}(h), \quad L_{g}: G \rightarrow G, \\
& h g=R_{g}(h), \quad R_{g}: G \rightarrow G, \\
& p g=\tilde{L}_{p}(g), \quad \tilde{L}_{p}: G \rightarrow P, \\
& p g=\tilde{R}_{g}(p), \quad \tilde{R}_{g}: P \rightarrow P .
\end{aligned}
$$

For $p \in P, \xi \in \mathfrak{g}$, and $v \in T_{p} P$,

$$
\begin{aligned}
p \xi:=\mathrm{d}_{\mathbb{1}} \tilde{L}_{p}(\xi)=\left.\frac{\mathrm{d}}{\mathrm{d} t}\right|_{t=0} p \exp (t \xi) & \in T_{p} P, \\
v g:=\mathrm{d}_{p} \tilde{R}_{g}(v) & \in T_{p g} P,
\end{aligned}
$$

and for $g, h \in G, \xi \in \mathfrak{g}$ and $v \in T_{h} G$,

$$
\begin{aligned}
g \xi:=\mathrm{d}_{\mathbb{1}} L_{g}(\xi)=\left.\frac{\mathrm{d}}{\mathrm{d} t}\right|_{t=0} g \exp (t \xi) & \in T_{g} G, \\
g v:=\mathrm{d}_{h} L_{g}(v) & \in T_{g h} G .
\end{aligned}
$$

We need to fix a certain inner product on $\mathfrak{g}$. The following lemma is the main reason that we restrict to compact Lie groups; its proof can be found in [Kna96, proposition 4.24, and the subsequent properties of the inner product and the induced metrics can be found in [Weh04, Remark A.3.

Lemma 1.12. Let $G$ be a compact Lie group. Then the Lie algebra $\mathfrak{g}$ admits an inner product which is invariant under the adjoint action of $G$ on $\mathfrak{g}$, that is, for all $\xi, \eta \in \mathfrak{g}$ and $g \in G$,

$$
\left\langle g \xi g^{-1}, g \eta g^{-1}\right\rangle=\langle\xi, \eta\rangle .
$$

This inner product satisfies, for all $\xi, \eta, \zeta \in \mathfrak{g}$,

$$
\langle[\xi, \eta], \zeta\rangle=\langle\xi,[\eta, \zeta]\rangle,
$$

and it can be rescaled in such a way that the associated norm $|\xi|=\sqrt{\langle\xi, \xi\rangle}$ satisfies, for all $\xi, \eta \in \mathfrak{g}$,

$$
|[\xi, \eta]| \leq|\xi| \cdot|\eta| .
$$

We will fix this inner product on $\mathfrak{g}$ throughout all of this text.

The inner product on $\mathfrak{g}$ also induces a metric on $T G$,

$$
\langle X, Y\rangle_{G}:=\left\langle g^{-1} X, g^{-1} Y\right\rangle_{\mathfrak{g}}, \forall X, Y \in T_{g} G,
$$


where $g^{-1} X$ is understood in the sense defined above. It is clear that with this metric left and right multiplication are isometries of $G$. If we denote by $\exp _{g}$ the exponential map with base point $g \in G$, then for all $\xi \in \mathfrak{g}$ and $g \in G$,

$$
\begin{aligned}
\exp _{g}(g \xi) & =g \exp (\xi), \\
\exp \left(g^{-1} \xi g\right) & =g^{-1} \exp (\xi) g .
\end{aligned}
$$

Moreover, the geodesics are 1-parameter subgroups,

$$
\exp ((s+t) \xi)=\exp (s \xi) \exp (t \xi)
$$

for all $s, t \in \mathbb{R}$ and $\xi \in \mathfrak{g}$.

Furthermore, we can define a geodesic distance on $G$,

$$
\mathrm{d}_{G}(g, h):=\inf \left\{|X|, X \in T_{g} G \text { and } h=\exp _{g}(X)\right\},
$$

which is invariant under left and right multiplication, and this can be used to define a metric on $C^{0}(U, G)$. For maps $u$ and $v$ from some domain $U$ to $G$, it will be denoted by

$$
\mathrm{d}(u, v):=\sup _{x \in U} \mathrm{~d}_{G}(u(x), v(x)),
$$

and this too will be invariant under left and right multiplication by continuous maps.

We also define a convex geodesic ball of radius $R$ around $\mathbb{1} \in G$ to be such that

(i) the exponential map is a bijection between $B_{R}(\mathbb{1}) \subseteq \mathfrak{g}$ and $B_{R}(\mathbb{1}) \subseteq G$,

(ii) and for all $g, h \in B_{R}(\mathbb{1})$ there is a unique minimal geodesic from $g$ to $h$ that lies entirely within $B_{R}(\mathbb{1})$.

For the existence of such balls see e.g. [GHL90, 2.89, 2.90], Moreover, because left multiplications are isometries of $G$, there exist convex geodesic balls of same radius around any $g \in G$.

Finally, we prove a small lemma on the (covariant) derivative of the adjoint action on $k$-forms.

Lemma 1.13. For $\tau \in \Omega^{k}(U, \mathfrak{g})$ and $u: U \rightarrow G$,

$$
\mathrm{d}\left(\operatorname{Ad}_{u} \tau\right)=\operatorname{Ad}_{u} \mathrm{~d} \tau+\left[\mathrm{d} u \cdot u^{-1} \wedge \operatorname{Ad}_{u} \tau\right] .
$$

Moreover, for the covariant derivative $\nabla$ induced by the canonical flat connection on the trivial vector bundle $U \times \mathfrak{g} \rightarrow U$,

$$
\nabla\left(u \tau u^{-1}\right)=u(\nabla \tau) u^{-1}+\left[\mathrm{d} u \cdot u^{-1}, u \tau u^{-1}\right] .
$$

Proof. For a fixed point $p \in U$ and some $X \in T U$,

$$
\begin{aligned}
\mathrm{d}_{p}\left(u \tau u^{-1}\right)(X) & =\mathrm{d}\left(\operatorname{Ad}_{u \cdot u(p)^{-1}}\left(u(p) \tau u(p)^{-1}\right)\right)(X) \\
& \stackrel{(1)}{=}\left(\mathrm{d}_{\mathbb{1}} \operatorname{Ad} \circ \mathrm{d}_{p}\left(u \cdot u(p)^{-1}\right) \cdot u(p) \tau u(p)^{-1}+\operatorname{Ad}_{u \cdot u(p)^{-1}} \mathrm{~d}_{p}\left(u(p) \tau u(p)^{-1}\right)\right)(X) \\
& \stackrel{(2)}{=}\left(\left[\mathrm{d}_{p} u \cdot u(p)^{-1}, u(p) \tau u(p)^{-1}\right]+u \mathrm{~d}_{p} \tau u^{-1}(p)\right)(X)
\end{aligned}
$$




$$
=\left(\left[\mathrm{d} u \cdot u^{-1}, u \tau u^{-1}\right]+u \mathrm{~d} \tau \cdot u^{-1}\right) X(p),
$$

where (1) follows from looking at Ad as a map on a product manifold, Ad : $G \times \mathfrak{g} \rightarrow \mathfrak{g}$, similar to what was done in the calculation of the gauge group action, lemma 1.10 , and (2) is just $\mathrm{d}_{\mathbb{1}} \operatorname{Ad}(\xi) \eta=\operatorname{ad}(\xi) \eta=[\xi, \eta]$.

We now use (1.23) to prove the second identity (1.24). With the canonical flat connection $\nabla^{E}=\mathrm{d}$, evaluating at vector fields $X_{j} \in T U$ and letting $\tilde{\tau}:=\tau\left(X_{1}, \ldots, X_{k}\right)$,

$$
\begin{gathered}
\nabla\left(u \tau u^{-1}\right)\left(X_{0}, \ldots, X_{k}\right)=\nabla_{X_{0}}^{E}\left(u \tilde{\tau} u^{-1}\right)-u \tau u^{-1}\left(\nabla_{X_{0}} X_{1}, \ldots, X_{k}\right) \\
-\cdots-u \tau u^{-1}\left(X_{1}, \ldots, \nabla_{X_{0}} X_{k}\right) \\
=\mathrm{d}\left(\operatorname{Ad}_{u} \tilde{\tau}\right) X_{0}-u \tau\left(\nabla_{X_{0}} X_{1}, \ldots, X_{k}\right) u^{-1} \\
-\cdots-u \tau\left(X_{1}, \ldots, \nabla_{X_{0}} X_{k}\right) u^{-1} \\
=\left[\mathrm{d} u\left(X_{0}\right) u^{-1}, u \tilde{\tau} u^{-1}\right]+u \mathrm{~d} \tilde{\tau}\left(X_{0}\right) u^{-1}-u \tau\left(\nabla_{X_{0}} X_{1}, \ldots, X_{k}\right) u^{-1} \\
-\cdots-u \tau\left(X_{1}, \ldots, \nabla_{X_{0}} X_{k}\right) u^{-1} \\
=\left[\mathrm{d} u \cdot u^{-1}, u \tau u^{-1}\right]\left(X_{0}, \ldots, X_{k}\right)+u \nabla \tau\left(X_{0}, \ldots, X_{k}\right) u^{-1} \\
=\left(\left[\mathrm{d} u \cdot u^{-1}, u \tau u^{-1}\right]+u(\nabla \tau) u^{-1}\right)\left(X_{0}, \ldots, X_{k}\right) .
\end{gathered}
$$

\subsection{An analytic interlude}

We now turn to the case when our objects may not be infinitely differentiable. The main reason for this is that $\Omega^{1}(M, \operatorname{ad}(P))$ has infinite dimension, and thus $\mathcal{A}(P)$ is an affine space with infinite dimensional vector space. Moreover, $\mathcal{G}(P)$ can be seen to be an infinite dimensional Lie group. Thus, we find ourselves in the realm of functional analysis, and we would like to work with Banach spaces.

In this section, our aim is to give the definitions and most relevant results on Sobolev spaces of sections of fibre bundles. For an introduction to the theory of Sobolev spaces on $\mathbb{R}^{n}$, we recommend [Eva10], chapter 5; for definitions and results on Sobolev spaces of functions on Riemannian manifolds, see Aub82, chapter 2. For Sobolev spaces of sections of vector bundles, see [Nic07, section 10.4.2. We mainly follow [Weh04], appendix B.

Let $(M, g)$ be a compact Riemannian manifold, $E \rightarrow M$ a vector bundle, and choose a bundle metric on $E$ and a compatible connection. We have shown that with these choices it is possible to define: a covariant derivative

$$
\nabla: \Omega^{k}(M, E) \rightarrow \Omega^{k+1}(M, E),
$$

given by the connection on $E$ and the Levi-Civita connection for the Riemannian metric $g$ as in 1.2 ; a pointwise inner product on $\Omega^{k}(M, E)$,

$$
\langle\alpha, \beta\rangle=*\langle\alpha \wedge * \beta\rangle: M \rightarrow \mathbb{R},
$$

given by the bundle metric and the Riemannian metric as in (1.7), which we then use to define $|\alpha|:=\sqrt{\langle\alpha, \alpha\rangle}$; and a way to integrate functions over $M$ given by the Riemannian metric, using the volume form as in 1.6 . 
Definition 1.14 (Sobolev space of sections of vector bundle). For $k \in \mathbb{N}_{0}$ and $1 \leq p<\infty$, the Sobolev space $W^{k, p}(M, E)$ of sections of the vector bundle $E \rightarrow M$ is defined as the completion of $\Gamma(E)$ with respect to the $W^{k, p}$-norm,

$$
\|\alpha\|_{k, p}=\sum_{j=1}^{k}\left\|\nabla^{j} \alpha\right\|_{p},
$$

where

$$
\left\|\nabla^{j} \alpha\right\|_{p}=\left(\int_{M}\left|\nabla^{j} \alpha\right|^{p}\right)
$$

Several properties of the usual Sobolev spaces on Euclidean space will generalize to Sobolev spaces of sections. From the definition, it is clear that $W^{k, p}(M, E)$ are Banach spaces, and therefore many important results from functional analysis are valid, in particular those from Appendix A. Moreover, this definition directly gives the density of smooth sections, which makes it possible to give proofs using approximating sequences of smooth sections, for which stronger results are valid; see, e.g., lemma 1.20 below. Other properties and results will be consequences of the following characterization:

Remark 1.15. Consider a finite bundle atlas $\left(U_{i}, \Phi_{i}\right)_{i=1}^{N}$ of $E \rightarrow M$, with $\Phi_{i}:\left.E\right|_{U_{i}} \rightarrow$ $V_{i} \times \mathbb{R}^{m}$ for $\phi: U_{i} \rightarrow V_{i} \subseteq \mathbb{R}^{n}$ a coordinate chart of $M$ and $m$ the rank of $E$. A section $\alpha \in \Gamma(E)$ which is locally $\alpha:\left.U_{i} \rightarrow E\right|_{U_{i}}$ is represented by $\Phi_{i *} \alpha: V_{i} \rightarrow \mathbb{R}^{m}$, or with $m$ components $\left(\Phi_{i *} \alpha\right)_{j}: V_{i} \rightarrow \mathbb{R}$. Then for $k \in \mathbb{N}_{0}$ and $1 \leq p<\infty$, the $W^{k, p}$-norm defined above is equivalent to

$$
\sum_{i=1}^{N} \sum_{j=1}^{m}\left\|\left(\Phi_{i *} \alpha\right)_{j}\right\|_{k, p\left(V_{i}\right)},
$$

where the $W^{k, p}$ norm on functions $\mathbb{R}^{n} \rightarrow \mathbb{R}$ is the usual one. Therefore $\alpha$ lies in $W^{k, p}(M, E)$ if and only if its local components $\left(\Phi_{i *} \alpha\right)_{j}$ are $W^{k, p}$-functions for all coordinate patches $i=1, \ldots, N$.

Note that it is essential that the base manifold is compact for the sum above to be finite and the norms equivalent.

From this characterization it also becomes clear that when $M$ is compact, the space $W^{k, p}(M, E)$ will not depend on the choices involved, as the $W^{k, p}$-norms induced will be equivalent. The choices were: the metric on $M$, the bundle metric on $E$, and the compatible connection on $E$.

Remark 1.16 (On the norm of a metric). In chapter 2, we will several times look at the $W^{k, \infty}$-norm of a Riemannian metric $g$. The metric is a tensor, $g \in \Gamma\left(\oplus^{2} T^{*} M\right)$, and therefore as a section of a vector bundle it makes sense to define its $W^{k, p}$-norm as above, but with respect to what metric on $M$ ? As we have noted, over a compact manifold all of the norms will be equivalent, so any fixed choice is valid. In chapter 2 we will mostly be working over open sets in Euclidean (half) space, and thus we may canonically choose the Euclidean metric in this case.

By far the most important result from the usual theory of Sobolev spaces which is also valid for sections of bundles is the Sobolev embeddings and estimates. Before stating the result, we need to define the norm on the spaces $C^{j}$ of continuous functions, for $j \in \mathbb{N}_{0}$. For $\alpha \in C^{0}\left(M, \otimes^{k} T^{*} M \otimes E\right)$ a $k$-form,

$$
\|\alpha\|_{\infty}:=\sup _{x \in M}|\alpha(x)|
$$


where $|\cdot|$ comes from the pointwise inner product defined on $\Omega^{k}(M, E)$ above; then for a section $\alpha \in C^{j}(M, E)$,

$$
\|\alpha\|_{j, \infty}:=\sup _{k \leq j}\left\|\nabla^{k} \alpha\right\|_{\infty}
$$

Finally, note that $W^{0, p}(M, E)$ is simply $L^{p}(M, E)$, and these $L^{p}$-spaces are also included in the following result, as $j=0$ is allowed.

A word on notation. We will denote the norm on the Sobolev space $W^{k, p}(M, E)$ over the Riemannian manifold $(M, g)$ as $\|\cdot\|_{g ; k, p(M)}$, and when the metric and the space are clear from context, they will be omitted from the norm.

Theorem 1.17 (Sobolev embeddings and estimates). Let $E \rightarrow M$ be a vector bundle over a compact Riemannian n-manifold, $j<k \in \mathbb{N}$ and $1 \leq p, q<\infty$.

(i) If $k-\frac{n}{p} \geq j-\frac{n}{q}$ then the inclusion

$$
W^{k, p}(M, E) \hookrightarrow W^{j, q}(M, E)
$$

is continuous, i.e., there exists a constant $C_{W}$ such that for $\alpha \in W^{k, p}(M, E)$,

$$
\|\alpha\|_{j, q} \leq C_{W}\|\alpha\|_{k, p}
$$

(ii) If $k-\frac{n}{p}>j-\frac{n}{q}$, this inclusion is a compact map.

(iii) Furthermore, if $k-\frac{n}{p}>j$, there is a continuous embedding $W^{k, p}(M) \hookrightarrow C^{j}(M)$, i.e., there exists a constant $C_{W}$ such that for $\alpha \in W^{k, p}(M, E)$,

$$
\|\alpha\|_{j, \infty} \leq C_{W}\|\alpha\|_{k, p} .
$$

Moreover, this inclusion is compact.

The generalization of the result from bounded domains in $\mathbb{R}^{n}$ to the case of vector bundles is straighforward using remark 1.15, see the discussion after Theorem A.2 in Weh04.

These embeddings will cause a few hypotheses to appear particularly frequently: the inclusion

$$
W^{k, p} \hookrightarrow C^{0}
$$

will lead to the condition $k p>n$; and the inclusion

$$
W^{1, p} \hookrightarrow L^{2 p}
$$

will lead to $p \geq \frac{n}{2}$. When looking at the Sobolev spaces of connections and gauge transformations, we will generally be working with the $W^{1, p}$ and $W^{2, p}$ spaces, and for $k=2$ the hypothesis $p>\frac{n}{2}$ guarantees both of these embeddings.

Of central importance to the results in this text is the Banach-Alaoglu theorem, which we state and use in the following form:

Theorem 1.18 (Banach-Alaoglu). Let $k \in \mathbb{N}$ and $1<p<\infty$, and let $E \rightarrow M$ be a vector bundle over a compact Riemannian manifold. Then every bounded sequence in $W^{k, p}(M, E)$ has a weakly convergent subsequence. 
The generalization from the result for bounded domains in $\mathbb{R}^{n}$ also follows from remark 1.15. As for the relation of this result to the usual Banach-Alaoglu theorem from functional analysis, note that Sobolev spaces are reflexive.

A recurrent argument used in proofs is to make use of the Banach-Alaoglu theorem to find a weakly convergent subsequence for a bounded sequence, and then use some compact Sobolev embedding to find a further subsequence which converges uniformly.

Finally, in order to deal with manifolds with boundary, we need the following result.

Theorem 1.19 (Trace theorem). Let $M$ be a compact Riemannian n-manifold and let $1 \leq p<\infty$. The restriction to the boundary $\partial M$ is a bounded linear operator $W^{1, p}(M) \rightarrow$ $L^{p}(\partial M)$.

Proof. See [Weh04], Theorem B.10.

This means that for any $h \in W^{1, p}(M)$,

$$
\left\|\left.h\right|_{\partial M}\right\|_{p(\partial M)} \leq C\|h\|_{1, p(M)} .
$$

In particular, any sequence of smooth functions that approximates $h$ in the $W^{k, p}(M)$-norm restricts to a sequence in the boundary that approximates $\left.h\right|_{\partial M}$ in the $L^{p}(\partial M)$-norm, since

$$
\left\|\left.\left(h_{j}-h\right)\right|_{\partial M}\right\|_{p(\partial M)} \leq c\left\|h_{j}-h\right\|_{1, p(M)} \leq c\left\|h_{j}-h\right\|_{k, p(M)} \rightarrow 0 .
$$

Using the trace theorem and approximating sequences, we can generalize Stokes's theorem to the non-smooth case. We state and prove this as a lemma, and make implicit use of it whenever we use Stokes's theorem.

Lemma 1.20 (Sobolev Stokes's Lemma). Let $M$ be a compact $n$-dimensional manifold with boundary, $E \rightarrow M$ a trivial vector bundle and $\alpha \in W^{k, p}\left(M, \otimes^{n-1} T^{*} M \otimes E\right)$ an $(n-1)$-form. Then

$$
\left.\int_{\partial M} \alpha\right|_{\partial M}=\int_{M} \mathrm{~d} \alpha
$$

Proof. We can approximate $\alpha$ in the $W^{1, p}(M)$-norm by a sequence $\left(\alpha_{j}\right)_{j \in \mathbb{N}} \subseteq \Omega^{n-1}(M, E)$ of smooth forms, and using the trace theorem $\left(\left.\alpha_{j}\right|_{\partial M}\right) \subseteq \Omega^{n-1}(\partial M, E)$ also approximates $\left.\alpha\right|_{\partial M}$ in the $L^{p}(\partial M)$-norm. Then, because of the triviality of the bundle, Stokes's theorem can be applied to each component of the smooth forms,

$$
\int_{M} \mathrm{~d} \alpha=\lim _{j \rightarrow \infty} \int_{M} \mathrm{~d} \alpha_{j}=\left.\lim _{j \rightarrow \infty} \int_{\partial M} \alpha_{j}\right|_{\partial M}=\left.\int_{\partial M} \alpha\right|_{\partial M}
$$

Finally, we will make use of a few product inequalities, but we state them in appendix A so as not to clutter the more important results here.

Let us shift our focus now to maps between manifolds, say $M$ and $X$. Suppose that $M$ is a compact $n$-dimensional manifold, and $X$ is an $\ell$-manifold, and fix on $X$ an embedding $\Phi: X \rightarrow \mathbb{R}^{2 \ell+1}$, an atlas $\left(U_{\alpha}, \phi_{\alpha}\right)_{\alpha \in A}$, and a metric. We then get the following two results.

Proposition 1.21. Let $M$ and $X$ be as above, and let $k \in \mathbb{N}$ and $1 \leq p<\infty$ be such that $k p>n$. For $u \in C^{0}(M, X)$ the following are equivalent: 
(i) $\phi_{\alpha} \circ u \in W^{k, p}\left(u^{-1}\left(U_{\alpha}, \mathbb{R}^{\ell}\right)\right.$ for all $\alpha \in A$,

(ii) $\Phi \circ u \in W^{k, p}\left(M, \mathbb{R}^{2 \ell+1}\right)$,

(iii) $u=\exp _{s}(V)$ for some $s \in C^{\infty}(M, X)$ and $V \in W^{k, p}\left(M, s^{*} T X\right)$.

In case $X=G$ is a Lie group with Lie algebra $\mathfrak{g}$, the last item can be reformulated as $u=s \cdot \exp (\xi)$ for $s \in C^{\infty}(M, G)$ and $\xi \in W^{k, p}(M, \mathfrak{g})$, and there is another equivalence,

(iv) $u^{-1} \mathrm{~d} u \in W^{k-1, p}\left(M, T^{*} M \otimes \mathfrak{g}\right)$.

Proposition 1.22. For a sequence $\left(u_{i}\right)_{i \in \mathbb{N}}$ and some $u$, all of which satisfy the equivalent conditions in the previous proposition, and under the same assumptions, the following are equivalent:

(i) $u_{i}$ converges to $u$ in the $C^{0}$-topology and $\phi_{\alpha} \circ u_{i}$ converges to $\phi_{\alpha} \circ u$ with respect to the $W^{k, p}$-norm for all $\alpha \in A$,

(ii) $\Phi \circ u_{i}$ converges to $\Phi \circ u$ with respect to the $W^{k, p}$-norm,

(iii) there exist $s \in C^{\infty}(M, X), V \in W^{k, p}\left(M, s^{*} T X\right)$, and for sufficiently large $i \in \mathbb{N}$ there are $V_{i} \in W^{k, p}\left(M, s^{*} T X\right)$ such that $u=\exp _{s}(V), u_{i}=\exp _{s}\left(V_{i}\right)$ and the $V_{i}$ converge to $V$ in the $W^{k, p}$-norm,

and in case $X=G$ is a Lie group with Lie algebra $\mathfrak{g}$, once again the last item can be reformulated with $u=s \cdot \exp (\xi), u_{i}=s \cdot \exp \left(\xi_{i}\right)$ and $\xi_{i} \rightarrow \xi$ in $W^{k, p}(M, \mathfrak{g})$, and there is another equivalence,

(iv) $u_{i}$ converges to $u$ in the $C^{0}$-topology and $u_{i}^{-1} \mathrm{~d} u_{i}$ converges to $u^{-1} \mathrm{~d} u$ in $W^{k-1, p}\left(M, T^{*} M \otimes\right.$ $\mathfrak{g})$.

Proof. See Weh04, lemmata B.5 and B.7.

A key lemma in the proof of the equivalences in proposition 1.22 is the following, which will also be used elsewhere in this text and for that reason we state it. This lemma is the reason for the assumption $k p>n$ in the previous results, and this hypothesis is needed here because of the embedding $W^{k, p} \hookrightarrow C^{0}$.

Lemma 1.23. Let $U \subseteq M$ be an open subset of a compact $n$-manifold, $1 \leq p<\infty$ and $k, m, N \in \mathbb{N}$ such that $k p>n$. If $f \in C^{k}\left(V \subseteq \mathbb{R}^{m}, \mathbb{R}^{N}\right)$, then composition with $f$ is a continuous map, that is

$$
\begin{aligned}
W^{k, p}(U, V) & \longrightarrow W^{k, p}\left(U, \mathbb{R}^{N}\right) \\
u & \mapsto f \circ u .
\end{aligned}
$$

Proof. See [Weh04], lemma B.8. This result too is stated there for bounded domains in $\mathbb{R}^{n}$ and then generalizes via remark 1.15 .

At last, we may give the definitions for Sobolev spaces of maps of manifolds and of sections of fibre bundles.

Definition 1.24 (Sobolev space of maps of manifolds). For $M$ and $X$ manifolds as described above, the Sobolev space $W^{k, p}(M, X)$ is given as a set by functions $u \in C^{0}(M, X)$ such that $u$ satisfies the equivalent statements of lemma 1.21 , and the topology on this space is given by defining its convergent sequences using the equivalent statements of lemma 1.22 . 
Definition 1.25 (Sobolev space of sections of fibre bundles). Let $X \hookrightarrow F \stackrel{\pi}{\longrightarrow} M$ be a fibre bundle, and fix a bundle atlas $\left(U_{\alpha}, \tau_{\alpha}\right)_{\alpha \in A}$. In every local trivialization $\pi \times \tau_{\alpha}: \pi^{-1}\left(U_{\alpha}\right) \rightarrow$ $U_{\alpha} \times X$ a section $u$ is represented by $\tau_{\alpha} \circ u: U_{\alpha} \rightarrow X$. Then define the Sobolev space $W^{k, p}(M, F)$ of sections of this fibre bundle to consist of all sections $u$ such that

$$
\tau_{\alpha} \circ u \in W^{k, p}\left(U_{\alpha}, X\right)
$$

for all $\alpha \in A$, following the definition above for maps of manifolds. The topology is once again given by the equivalent conditions in proposition 1.22 via convergence in a bundle atlas.

We make a few observations. First, proposition 1.21 shows that $W^{k, p}(M, X)$ is independent of the choice of atlas (used in condition $(i)$ ), embedding (used in condition $(i i)$ ) and metric (used in condition (iii)), and therefore it is well defined for $k p>n$. Moreover, the definition via embeddings makes it possible to extend the Sobolev embeddings in theorem 1.17 to the Sobolev spaces defined above.

\section{Partial differential equations and elliptic operators}

When working with partial differential equations (PDEs), there are four main aspects of solutions that one is usually concerned with in an abstract context: existence, uniqueness, regularity and estimates. Existence, of course, has to do with whether there is a solution to the given problem in the chosen domain, which might be some $C^{k}$ or Sobolev space; if a solution exists, it is often good to know whether it is unique, at least up to some equivalence class (up to a measure zero set, for instance); regularity means, roughly, the degree of smoothness of a solution; and estimates, for our purposes, are very useful tools in proofs.

Throughout this text, the main PDE we will be concerned with is the Yang-Mills equation. However, in the proofs of the main theorems we will need to quote results on two other equations: the Neumann problem, and the $\mathrm{d}+\mathrm{d}^{*}$ operator.

For the Yang-Mills equation, we will need a very important regularity result (theorem 1.39, which will be crucial in the proof of the strong compactness theorem 3.9. This will be discussed in more detail in the next section, when discussing non-smooth Yang-Mills connections.

For the Neumann problem, we will need results on existence and uniqueness, as well as an estimate; these will be used only once, in the proof of theorem 2.2, and can be found in appendix A.

For the $\mathrm{d}+\mathrm{d}^{*}$ operator, we will need the following estimate, which will be used twice in chapter 2 , in the motivation and proof of theorem 2.2 .

Theorem 1.26 ([Weh04, theorem 5.1). Let $M$ be a compact manifold with (possibly empty) boundary. For $1<p<\infty$, if $A \in W^{1, p}\left(M, T^{*} M\right)$ satisfies $\left.* A\right|_{\partial M}=0$ and $H^{1}(M ; \mathbb{R})=0$, then there is a constant $C$ such that

$$
\|A\|_{1, p} \leq C\left(\|\mathrm{~d} A\|_{p}+\left\|\mathrm{d}^{*} A\right\|_{p}\right) .
$$

Moreover, this constant depends $W^{1, \infty}$-continuously on the metric on $M$. This is also valid for 1-forms with values in a finite dimensional vector space. 
All of these results are proved individually and in great detail in the book [Weh04, and indeed have many chapters dedicated to them. However, it is worth commenting that all of the operators associated to these equations are part of a class of special operators with good properties, called elliptic operators. Indeed, it is often the case that saying that an operator is elliptic is enough justification for certain results. For instance, in Uhlenbeck's original paper [Uhl82], after the statement of Theorem 1.3, she writes: 8

"Regularity of solutions of Yang-Mills equations for connections $A \in \mathcal{A}^{1, p}, 2 p \geq$ $\operatorname{dim} M$ follows rather easily from [the gauge fixing] theorem. (...) The system of equations (...) is uniformly elliptic. Now standard techniques apply."

Another example, from Donaldson and Kronheimer's book [DK97], p. 55:

"The operator $\mathrm{d}+\mathrm{d}^{*}$ is elliptic, its kernel decomposes according to degree and so if, as we suppose, $H^{1}(M)$ is zero, all the 1-forms are orthogonal to the kernel. So elliptic theory gives inequalities

$$
\|A\|_{k, 2} \leq \text { const. }\left(\left\|\mathrm{d}^{*} A\right\|_{k-1,2}+\|\mathrm{d} A\|_{k-1,2}\right) .
$$

While we have been discussing above the case of a closed base manifold, similar ideas can be applied on manifolds with boundary or on complete manifolds, given appropriate boundary or decay conditions."

We will not delve into elliptic theory, as that is beyond the scope of this work, but we point to references. For elliptic theory on compact manifolds, a good introduction which avoids doing too much analysis can be found in [Nic07], chapter 10; a more succinct overview which delves into the analytical aspects is [Wel80], chapter IV. For elliptic boundary value problems (i.e. on manifolds with boundary), see [Sch95] or [H0̈7], section 20.1.

\subsection{Analysis in gauge theory}

With all of the analytical machinery defined, we can turn again to gauge theory. In all that follows, let $P \rightarrow M$ be a principal $G$-bundle over a compact Riemannian $n$-manifold.

Definition 1.27 (Sobolev space of connections). Fix a smooth reference connections $\tilde{A} \in$ $\mathcal{A}(P)$, and note that it gives a covariant derivative $\nabla^{\tilde{A}}$ on the associated vector bundle $\operatorname{ad}(P) \rightarrow M$. Then we define the (affine) Sobolev space of connections as

$$
\mathcal{A}^{k, p}(P):=\tilde{A}+W^{k, p}\left(M, T^{*} M \otimes \operatorname{ad}(P)\right),
$$

modelled after the Sobolev space of sections $W^{k, p}\left(M, T^{*} M \otimes \operatorname{ad}(P)\right) .9$ which is a vector space.

Once again, note that since $M$ is compact, $\mathcal{A}^{k, p}(P)$ will not depend on the choices of Riemannian metric and reference connection, even though the norm on $W^{k, p}\left(M, T^{*} M \otimes\right.$ $\operatorname{ad}(P))$ does depend on these choices.

\footnotetext{
${ }^{8}$ The mathematical notation in the quotes was changed to agree with the one fixed in this work, so as not to cause confusion.

${ }^{9}$ See definition 1.14 .
} 
Recalling the definitions given in subsection 1.2.1 in a local trivialization $\Phi=\pi \times \phi$ : $\pi^{-1}(U) \rightarrow U \times G$ over some $U \subseteq M$ we have a natural reference connections $\tilde{A}=\phi^{-1} \mathrm{~d} \phi$ and can represent a connection by $A=\tilde{A}+\alpha$ for $\alpha \in \Omega^{1}(U, \mathfrak{g})$. The reference connection can be used to define $\mathcal{A}^{k, p}\left(\left.P\right|_{U}\right)$, and we affirm that the norm on $W^{k, p}\left(U, T^{*} U \otimes \operatorname{ad}\left(\left.P\right|_{U}\right)\right)$ is equal to the norm on $W^{k, p}\left(U, T^{*} U \otimes \mathfrak{g}\right)$. Thus we are able to locally define the Sobolev space of connections as

$$
\mathcal{A}^{k, p}(U):=W^{k, p}\left(U, T^{*} U \otimes \mathfrak{g}\right),
$$

such that

$$
\mathcal{A}^{k, p}\left(\left.P\right|_{U}\right)=\phi^{-1} \mathrm{~d} \phi+\mathcal{A}^{k, p}(U) .
$$

Definition 1.28 (Sobolev space of gauge transformations). For $k p>n$, we define the Sobolev space of gauge transformations as the Sobolev space of sections of the bundle $\operatorname{Ad}(P) \rightarrow M$

$$
\mathcal{G}^{k, p}(P):=W^{k, p}(M, \operatorname{Ad}(P)) .
$$

Similar to the smooth case, this is naturally isomorphic to the Sobolev space of $G$ equivariant maps $W^{k, p}(P, G)^{G}$, and from proposition 1.21, a map $u \in W^{k, p}(P, G)^{G}$ can be written as $u=s \cdot \exp (\xi)$ for $s \in C^{\infty}(P, G)^{G}$ a smooth gauge transformation, and $\xi \in$ $W^{k, p}(P, \mathfrak{g})^{G}$. On a local trivialization over $U \subseteq M$, a gauge transformation is represented by a map $u: U \rightarrow G$, and thus locally we can identify $\mathcal{G}^{k, p}\left(\left.P\right|_{U}\right)$ with

$$
\mathcal{G}^{k, p}(U):=W^{k, p}(U, G),
$$

and furthermore proposition 1.21 will yield

$$
u^{-1} \mathrm{~d} u \in W^{k-1, p}\left(U, T^{*} U \otimes \mathfrak{g}\right)=\mathcal{A}^{k-1, p}(U)
$$

for $u \in \mathcal{G}^{k, p}(U)$.

Just as we defined the Yang-Mills functional to be an $L^{2}$-energy, we may generalize this and define an $L^{q}$-energy of a connection $A \in \mathcal{A}(P)$, for $1 \leq q<\infty$,

$$
\mathcal{E}_{q}(A):=\int_{M}\left|F_{A}\right|^{q}=\left\|F_{A}\right\|_{q}^{q} .
$$

We may extend these functionals to Sobolev spaces of connections, and they will be well behaved.

Lemma 1.29. When $\frac{n}{2} \leq q<\infty, \mathcal{E}_{q}$ is a continuous functional on $\mathcal{A}^{1, q}(P)$, and for every smooth reference connection $\tilde{A}$ there exists a constant $C$ such that for all $A=\tilde{A}+\alpha \in$ $\mathcal{A}^{1, p}(P)$

$$
\mathcal{E}_{q}(A)^{\frac{1}{q}} \leq \mathcal{E}_{q}(\tilde{A})^{\frac{1}{q}}+2\|\alpha\|_{1, q}+C\|\alpha\|_{1, q}^{2} .
$$

Proof. The curvature of $A=\tilde{A}+\alpha$ is

$$
\begin{aligned}
F_{A} & =\mathrm{d}(\tilde{A}+\alpha)+\frac{1}{2}[(\tilde{A}+\alpha) \wedge(\tilde{A}+\alpha)] \\
& =\mathrm{d} \tilde{A}+\mathrm{d} \alpha+\frac{1}{2}([\tilde{A} \wedge \tilde{A}]+[\alpha \wedge \alpha]+2[\tilde{A} \wedge \alpha])
\end{aligned}
$$

\footnotetext{
${ }^{10}$ See definition 1.25
} 


$$
\begin{aligned}
& =F_{\tilde{A}}+\mathrm{d} \alpha+[\tilde{A} \wedge \alpha]+\frac{1}{2}[\alpha \wedge \alpha] \\
& =F_{\tilde{A}}+\mathrm{d}_{\tilde{A}} \alpha+\frac{1}{2}[\alpha \wedge \alpha] .
\end{aligned}
$$

From (1.4), we know that for any $X, Y \in T M$

$$
\mathrm{d}_{\tilde{A}} \alpha(X, Y)=\nabla^{\tilde{A}} \alpha(X, Y)-\nabla^{\tilde{A}} \alpha(Y, X),
$$

which implies $\left|\mathrm{d}_{\tilde{A}} \alpha\right| \leq 2\left|\nabla^{\tilde{A}} \alpha\right|$.

Writing $\alpha=\alpha_{i} \mathrm{~d} x_{i}$ on a local frame $\left\{\partial x_{i}\right\}$,

$$
[\alpha \wedge \alpha]=\sum_{i, j}\left[\alpha_{i}, \alpha_{j}\right] \mathrm{d} x_{i} \mathrm{~d} x_{j}=\sum_{i<j} 2\left[\alpha_{i}, \alpha_{j}\right] \mathrm{d} x_{i} \mathrm{~d} x_{j}
$$

and so

$$
\left|\frac{1}{2}[\alpha \wedge \alpha]\right|^{2}=\sum_{i<j}\left|\left[\alpha_{i}, \alpha_{j}\right]\right|^{2} \stackrel{(*)}{\leq} \sum_{i<j}\left|\alpha_{i}\right|^{2}\left|\alpha_{j}\right|^{2} \leq \sum_{i, j}\left|\alpha_{i}\right|^{2}\left|\alpha_{j}\right|^{2}=|\alpha|^{4},
$$

where we used (1.21) in $(*)$, and then

$$
\left\|\frac{1}{2}[\alpha \wedge \alpha]\right\|_{q}=\left(\int\left|\frac{1}{2}[\alpha \wedge \alpha]\right|^{q}\right)^{\frac{1}{q}} \leq\left(\int|\alpha|^{2 q}\right)^{\frac{1}{q}}=\|\alpha\|_{2 q}^{2} .
$$

Finally,

$$
\begin{aligned}
\left|\mathcal{E}_{q}(A)^{\frac{1}{q}}-\mathcal{E}_{q}(\tilde{A})^{\frac{1}{q}}\right| & =\left|\left\|F_{\tilde{A}}+\mathrm{d}_{\tilde{A}} \alpha+\left.\frac{1}{2}[\alpha \wedge \alpha]\right|_{q}-\right\| F_{\tilde{A}} \|_{q}\right| \\
& \leq\left|\left\|\mathrm{d}_{\tilde{A}} \alpha\right\|_{q}+\left\|\frac{1}{2}[\alpha \wedge \alpha]\right\|_{q}\right| \\
& \leq 2\left\|\nabla^{\tilde{A}} \alpha\right\|_{q}+\|\alpha\|_{2 q}^{2} \\
& \leq 2\|\alpha\|_{1, q}+C_{W}\|\alpha\|_{1, q}^{2},
\end{aligned}
$$

where the last inequality follows because of the embedding $W^{1, q} \hookrightarrow L^{2 p}$ which holds with the assumption $q \geq \frac{n}{2}$.

Locally, the energy of a connection $A \in \mathcal{A}^{1, q}(U)$ is denoted the same way and given by

$$
\mathcal{E}_{q}(A)=\left\|F_{A}\right\|_{q(U)}^{q},
$$

where $F_{A} \in L^{q}\left(U, \Lambda^{2} T^{*} U \otimes \mathfrak{g}\right)$ is the local representative of the curvature. Then the estimate in the previous lemma becomes

$$
\mathcal{E}_{q}(A)^{\frac{1}{q}}=\left\|F_{A}\right\|_{q} \leq 2\|A\|_{1, q}+C\|A\|_{1, q}^{2} .
$$

We already know that the group of gauge transformations is indeed a group; we now prove that the group operations are continuous, and therefore the $\mathcal{G}^{k, p}$ are topological groups, for $k p>n$. 
Lemma 1.30. Let $k \in \mathbb{N}$ and $1 \leq p<\infty$ be such that $k p>n$; then group multiplication and inversion are continuous maps on $\mathcal{G}^{k, p}(P)$.

Proof. Let $\left(U_{\alpha}, \tau_{\alpha}\right)_{\alpha \in A}$ be a bundle atlas of $\operatorname{Ad}(P) \rightarrow M$. By definition, a gauge transformation $u \in \mathcal{G}^{k, p}(P)$ is a continuous section of this bundle such that for all $\alpha \in A$, $u_{\alpha}:=\tau_{\alpha} \circ u: U_{\alpha} \rightarrow G$ is in $W^{k, p}\left(U_{\alpha}, G\right)$. If $\left(V_{\beta}, \phi_{\beta}\right)_{\beta \in B}$ is an atlas of $G$, then this means that $\phi_{\beta} \circ u_{\alpha} \in W^{k, p}\left(u_{\alpha}^{-1}\left(V_{\beta}\right), \mathbb{R}^{\ell}\right)$, for all $\beta \in B$ and $\ell$ the dimension of $G$. We will prove the continuity of the inversion map, and the multiplication will be analogous.

Let $i: G \rightarrow G$ be the inversion map, $i(g)=g^{-1}$. Then $u^{-1}$ can be defined by $\left(u^{-1}\right)_{\alpha}=$ $i \circ u_{\alpha}$ for all $\alpha \in A$. We would like to show that $\left(u^{-1}\right)_{\alpha} \in W^{k, p}\left(U_{\alpha}, G\right)$. For $\gamma \in B$,

$$
\phi_{\gamma} \circ\left(u^{-1}\right)_{\alpha}=\phi_{\gamma} \circ i \circ u_{\alpha}=\left(\phi_{\gamma} \circ i \circ \phi_{\beta}^{-1}\right) \circ\left(\phi_{\beta} \circ u_{\alpha}\right) .
$$

Now we can use Lemma 1.23 with $f=\phi_{\gamma} \circ i \circ \phi_{\beta}^{-1} \in C^{\infty}\left(\phi_{\beta}\left(V_{\beta}\right) \subseteq \mathbb{R}^{\ell}, \mathbb{R}^{\ell}\right)$ to conclude that $\phi_{\gamma} \circ\left(u^{-1}\right)_{\alpha} \in W^{k, p}\left(\left(u^{-1}\right)_{\alpha}^{-1}\left(V_{\gamma}\right), \mathbb{R}^{\ell}\right)$. Since $\alpha \in A$ and $\gamma \in B$ were arbitrary, this proves $u^{-1} \in \mathcal{G}^{k, p}(P)$.

Moreover, the gauge action on the appropriate Sobolev space of connections is continuous.

Lemma 1.31. Let $k \in \mathbb{N}$ and $1 \leq p<\infty$ be such that $k p>n$; then the gauge action

$$
\begin{aligned}
\mathcal{G}^{k, p}(P) \times \mathcal{A}^{k-1, p}(P) & \rightarrow \mathcal{A}^{k-1, p}(P) \\
(u, A) & \mapsto u^{*} A
\end{aligned}
$$

is a continuous map. Moreover, for every trivializing neighbourhood $U \subseteq M$ there is a constant $C$ such that for $u \in \mathcal{G}^{k, p}(U)$ and $A \in \mathcal{A}^{k-1, p}(U)$ the following holds:

$$
\left\|u^{*} A\right\|_{k-1, p} \leq\left\|u^{-1} \mathrm{~d} u\right\|_{k-1, p}+C\|A\|_{k-1, p}\left(1+\left\|u^{-1} \mathrm{~d} u\right\|_{k-2,2 p}\right)^{k-1} .
$$

Proof. First, note that it suffices to prove continuity of the action on an arbitrary trivializing neighbourhood, say $U \subseteq M$. We wish to prove that for two sequences $\left(u_{i}\right) \subseteq \mathcal{G}^{k, p}(U)$ and $\left(A_{i}\right) \subseteq \mathcal{A}^{k-1, p}(U)$ converging to $u \in \mathcal{G}^{k, p}(U)$ and $A \in \mathcal{A}^{k-1, p}(U)$, respectively,

$$
\left(u_{i}\right)^{-1} A_{i} u_{i}+\left(u_{i}\right)^{-1} \mathrm{~d} u_{i}=u_{i}^{*} A_{i} \longrightarrow u^{*} A .
$$

We treat the cases $k=1$ and $k \geq 2$ separately. In both cases, note that by definition (lemma $1.22,\left(u_{i}\right)$ converges to $u$ in $C^{0}(U, G)$ and $\left(u_{i}\right)^{-1} \mathrm{~d} u_{i} \stackrel{(k-1, p)}{\longrightarrow} u^{-1} \mathrm{~d} u$.

For $k=1$, the inequality is simply

$$
\left\|u^{*} A\right\|_{p(U)} \leq\left\|u^{-1} \mathrm{~d} u\right\|_{p(U)}+\left\|u^{-1} A u\right\|_{p(U)},
$$

which is just the triangle inequality for the norm of the gauge action. Then when looking at $\left\|u^{*} A-u_{i}^{*} A_{i}\right\|_{p(U)}$, we have shown that the first term converges, and the second term

$$
\begin{aligned}
\left\|u^{-1} A u-u_{i}^{-1} A_{i} u_{i}\right\|_{p} & \leq\left\|u^{-1} A u-u^{-1} A_{i} u\right\|_{p}+\left\|u^{-1} A_{i} u-u_{i}^{-1} A_{i} u_{i}\right\|_{p} \\
& \leq\left\|A-A_{i}\right\|_{p}+\left\|\operatorname{Ad}_{u}-\operatorname{Ad}_{u_{i}}\right\|\left\|A_{i}\right\|_{p}
\end{aligned}
$$


will also converge because of the $C^{0}$-convergence $u_{i} \rightarrow u$. For the second inequality, we used the invariante of the metric on $\mathfrak{g}$ under conjugation and the fact that $\operatorname{Ad}_{g}$ is a bounded linear operator on $\mathfrak{g}$.

For $k \geq 2$, once again writing

$$
\left\|u^{*} A\right\|_{k-1, p(U)} \leq\left\|u^{-1} \mathrm{~d} u\right\|_{k-1, p(U)}+\left\|u^{-1} A u\right\|_{k-1, p(U)},
$$

the first term is known to converge, and for the second term we use lemma 1.32 below with $\tau=A, \tau_{i}=A_{i}$ and $\ell=k-1$ to get convergence and an estimate which yields the desired inequality.

Lemma 1.32. Let $U \subseteq M$ be a trivializing neighbourhood of $P \rightarrow M, 0 \leq \ell \leq k$ be integers and $1 \leq p<\infty$ be such that $k p>n$ and $p \geq \frac{n}{2}$. Then the following holds.

Let $\left(u_{i}\right) \subseteq \mathcal{G}^{k, p}(U)$ and $\left(\tau_{i}\right) \subseteq W^{\ell, p}\left(\Lambda^{\ell} T^{*} U \otimes \mathfrak{g}\right)$ be sequences that converge to $u \in \mathcal{G}^{k, p}(U)$ and $\tau \in W^{\ell, p}\left(\Lambda^{\ell} T^{*} U \otimes \mathfrak{g}\right)$, respectively and in the appropriate topologies. Then

$$
\left(u_{i}\right)^{-1} \tau_{i} u_{i} \stackrel{\ell, p}{\longrightarrow} u^{-1} \tau u .
$$

Moreover, there exists a constant $C$ such that

$$
\left\|u^{-1} \tau u\right\|_{\ell, p} \leq C\|\tau\|_{\ell, p}\left(1+\left\|u^{-1} \mathrm{~d} u\right\|_{\ell-1,2 p}\right)^{\ell}
$$

Proof. The proof will go through by induction on $\ell$.

For $\ell=0$, the estimate is provided by the invariance of the metric on $\mathfrak{g}$ under conjugation and is simply

$$
\left\|u^{-1} \tau u\right\|_{p}=\|\tau\|_{p}
$$

Convergence follows from the $C^{0}$ convergence of the $u_{i}$ (lemma 1.22).

Assume the lemma to hold for $\ell-1 \geq 0$. The case $\ell=0$ already provides convergence for the $L^{p}$ term in the norm and it remains to show that the derivative, which we write using (1.24) and $\mathrm{d} u^{-1} \cdot u=-u^{-1} \mathrm{~d} u$,

$$
\nabla\left(u_{i}^{-1} \tau_{i} u_{i}\right)=u_{i}^{-1} \nabla \tau_{i} u_{i}-\left[u_{i}^{-1} \mathrm{~d} u_{i}, u_{i}^{-1} \tau_{i} u_{i}\right],
$$

converges to $\nabla\left(u^{-1} \tau u\right)$ in the $W^{\ell-1, p_{-}}$norm. The first term in the derivative can be seen to converge from the induction hypothesis, using the lemma for $\nabla \tau$ and $\ell-1$. For the second term, the Lie bracket, note the calculation below,

$$
\begin{aligned}
& \left\|\left[u_{i}^{-1} \mathrm{~d} u_{i}, u_{i}^{-1} \tau_{i} u_{i}\right]-\left[u^{-1} \mathrm{~d} u, u^{-1} \tau u\right]\right\|_{\ell-1, p} \\
& =\left\|\left[u_{i}^{-1} \mathrm{~d} u_{i}-u^{-1} \mathrm{~d} u, u_{i}^{-1} \tau_{i} u_{i}\right]+\left[u^{-1} \mathrm{~d} u, u_{i}^{-1} \tau_{i} u_{i}-u^{-1} \tau u\right]\right\|_{\ell-1, p} \\
& \leq\left\|\left[u_{i}^{-1} \mathrm{~d} u_{i}-u^{-1} \mathrm{~d} u, u_{i}^{-1} \tau_{i} u_{i}\right]\right\|_{\ell-1, p}+\left\|\left[u^{-1} \mathrm{~d} u, u_{i}^{-1} \tau_{i} u_{i}-u^{-1} \tau u\right]\right\|_{\ell-1, p} \\
& \stackrel{(1)}{\leq}\left\|\left|u_{i}^{-1} \mathrm{~d} u_{i}-u^{-1} \mathrm{~d} u\right| \cdot\left|u_{i}^{-1} \tau_{i} u_{i}\right|\right\|_{\ell-1, p}+\left\|\left|u^{-1} \mathrm{~d} u\right| \cdot\left|u_{i}^{-1} \tau_{i} u_{i}-u^{-1} \tau u\right|\right\|_{\ell-1, p} \\
& \stackrel{(2)}{\leq}\left\|u_{i}^{-1} \mathrm{~d} u_{i}-u^{-1} \mathrm{~d} u\right\|_{\ell-1,2 p}\left\|u_{i}^{-1} \tau_{i} u_{i}\right\|_{\ell-1,2 p} \\
& +\left\|u^{-1} \mathrm{~d} u\right\|_{\ell-1,2 p}\left\|u_{i}^{-1} \tau_{i} u_{i}-u^{-1} \tau u\right\|_{\ell-1,2 p}
\end{aligned}
$$




$$
\begin{aligned}
\stackrel{(3)}{\leq} C_{W} \underbrace{\left\|u_{i}^{-1} \mathrm{~d} u_{i}-u^{-1} \mathrm{~d} u\right\|_{\ell, p}}_{\stackrel{(4)}{\longrightarrow} 0} \underbrace{\left\|u_{i}^{-1} \tau_{i} u_{i}\right\|_{\ell-1,2 p}}_{\text {const. }} \\
+C_{W}^{\prime} \underbrace{\stackrel{\stackrel{(5)}{\longrightarrow} \mathrm{d} u \|_{\ell, p}}{\left\|u_{i}^{-1} \tau_{i} u_{i}-u^{-1} \tau u\right\|_{\ell-1,2 p}}}_{\stackrel{(5)}{\longrightarrow}\left\|u^{-1} \tau u\right\|_{\ell-1,2 p}} \rightarrow 0,
\end{aligned}
$$

where (1) follows from 1.20); (2) is lemma A.8 with $r=s=2 p$; (3) is the Sobolev embedding $W^{\ell, p} \hookrightarrow W^{\ell-1,2 p}$ which is valid for $p \geq \frac{n}{2}$; (4) follows from Lemma 1.22 noting that $\ell \leq k-1 ;(5)$ and (6) follow taking the lemma with $\ell-1$ (which is valid by induction hypothesis) but with $(k-1,2 p)$ instead of $(k, p)$, since by the same embedding $W^{\ell, p} \hookrightarrow$ $W^{\ell-1,2 p}$, the original sequence $\left(\tau_{i}\right)$ also converges in $W^{\ell-1,2 p}$-norm and $\left(u_{i}\right)$ also converges in $\mathcal{G}^{k-1,2 p}$. This proves convergence for $\ell$ from $\ell-1$.

For the estimate, assume it valid for $\ell-1$. Then, denoting by $C$ all constants which do not come from a Sobolev estimate, and by $C_{W}$ all of the ones that do,

$$
\begin{aligned}
\left\|u^{-1} \tau u\right\|_{\ell, p} \leq\left\|u^{-1} \tau u\right\|_{p}+\left\|\nabla\left(u^{-1} \tau u\right)\right\|_{\ell-1, p} \\
\quad \stackrel{(1)}{\leq}\|\tau\|_{p}+\left\|u^{-1} \nabla \tau u\right\|_{\ell-1, p}+\left\|u^{-1} \mathrm{~d} u\right\|_{\ell-1,2 p}\left\|u^{-1} \tau u\right\|_{\ell-1,2 p} \\
\quad \stackrel{(2)}{\leq}\|\tau\|_{p}+C\|\nabla \tau\|_{\ell-1, p}\left(1+\left\|u^{-1} \mathrm{~d} u\right\|_{\ell-2,2 p}\right)^{\ell-1} \\
\quad+\left\|u^{-1} \mathrm{~d} u\right\|_{\ell-1,2 p} C\|\tau\|_{\ell-1,2 p}\left(1+\left\|u^{-1} \mathrm{~d} u\right\|_{\ell-2,4 p}\right)^{\ell-1} \\
\leq C\|\tau\|_{\ell, p}\left(1+\left\|u^{-1} \mathrm{~d} u\right\|_{\ell-1,2 p}\right)^{\ell-1} \\
\quad+\left(1+\left\|u^{-1} \mathrm{~d} u\right\|_{\ell-1,2 p}\right) C_{W}\|\tau\|_{\ell, p}\left(1+C_{W}\left\|u^{-1} \mathrm{~d} u\right\|_{\ell-1,2 p}\right)^{\ell-1} \\
\leq C\|\tau\|_{\ell, p}\left(1+\left\|u^{-1} \mathrm{~d} u\right\|_{\ell-1,2 p}\right)^{\ell}
\end{aligned}
$$

where (1) follows from arguments similar to those already used for the convergence above, (2) is the induction hypothesis, and the Sobolev estimates for $W^{\ell, p} \hookrightarrow W^{\ell-1,2 p}$ and $W^{\ell-1,2 p} \hookrightarrow$ $W^{\ell-2,4 p}$ hold due to $p \geq \frac{n}{2}$.

The following results will be critical for proving the weak and strong compactness theorems.

Lemma 1.33. Let $k \in \mathbb{N}$ and $1 \leq p<\infty$ be such that $k p>n$ and $p>\frac{n}{2}$. Let $\left(A^{i}\right)_{i \in \mathbb{N}} \subseteq$ $\mathcal{A}^{k-1, p}(P)$ and $\left(u^{i}\right)_{i \in \mathbb{N}} \subseteq \mathcal{G}^{k, p}(P)$ be two sequences such that both $\left\|A^{i}\right\|_{k-1, p}$ and $\left\|u^{i *} A^{i}\right\|_{k-1, p}$ are uniformly bounded. Then the following holds:

(i) For every trivialization over some domain $U_{\alpha} \subseteq M$, there is a uniform bound on $\left\|\left(u_{\alpha}^{i}\right)^{-1} \mathrm{~d} u_{\alpha}^{i}\right\|_{k-1, p\left(U_{\alpha}\right)}$.

(ii) There exists a subsequence of $\left(u^{i}\right)$ that converges in the $C^{0}$-topology to some limit in $\mathcal{G}^{k, p}(P)$.

Proof. See [Weh04, lemma A.8. 
Lemma 1.34. Let $1<p<\infty$ and $k \in \mathbb{N}_{0}$ such that $k p>n$ and $p \geq \frac{n}{2}$. Let $A, A^{\prime} \in \mathcal{A}^{k, p}(P)$. If there is a continuous gauge transformation $u$ such that $A^{\prime}=u^{*} A$, then $u \in \mathcal{G}^{k+1, p}(P)$.

Proof. Look at the local representatives on some chart $U \subseteq M$, such that $A, A^{\prime} \in \mathcal{A}^{k, p}(U)$ and $u \in C^{0}(U, G)$, then write $A^{\prime}=u^{-1} A u+u^{-1} \mathrm{~d} u$ (the gauge action looks the same locally, see lemma 1.11). In case $k=0$, it suffices to look at

$$
\left\|u^{-1} \mathrm{~d} u\right\|_{p} \leq\left\|A^{\prime}-u^{-1} A u\right\|_{p} \leq\left\|A^{\prime}\right\|_{p}+\|A\|_{p},
$$

where in the second inequality we used that the inner product on $\mathfrak{g}$ is Ad-invariant. Then $u^{-1} \mathrm{~d} u \in L^{p}\left(U, T^{*} U \otimes \mathfrak{g}\right)$, and so by proposition 1.21, $u \in \mathcal{G}^{1, p}(P)$.

For $k>1$, we will use the estimate in lemma 1.32 above. First, note that for all $j \leq k$,

$$
\left\|u^{-1} \mathrm{~d} u\right\|_{2^{j} p} \leq\left\|A^{\prime}\right\|_{2^{j} p}+\|A\|_{2^{j} p} \leq\left\|A^{\prime}\right\|_{k, p}+\|A\|_{k, p}
$$

since $k p>n>\frac{2^{j}-1}{2^{j}} n$. Then,

$$
\left\|u^{-1} \mathrm{~d} u\right\|_{k, p} \leq\left\|A^{\prime}\right\|_{k, p}+\left\|u^{-1} A u\right\|_{k, p},
$$

and from lemma 1.32 , $\left\|u^{-1} A u\right\|_{k, p}$ is bounded by $\|A\|_{k, p}$ and $\left\|u^{-1} \mathrm{~d} u\right\|_{k-1,2 p}$. The norm $\left\|A^{\prime}\right\|_{k, p}$ is finite by assumption, and we bound the second term as follows: we iterate the estimate above, using the embeddings

$$
W^{k, p} \hookrightarrow W^{k-1,2 p} \hookrightarrow W^{k-2,4 p} \hookrightarrow \cdots \hookrightarrow W^{k-j, 2^{j} p}
$$

to bound the $\left\|A^{\prime}\right\|$ term, which hold since $p \geq \frac{n}{2} \geq \frac{n}{2^{j}}$ for $j \geq 1$, and using lemma 1.32 to bound the $\left\|u^{-1} A u\right\|$ term. This process will finally end with $\left\|u^{-1} \mathrm{~d} u\right\|_{k, p}$ bounded by a finite amount of terms $\left\|A^{\prime}\right\|_{k, p}$ and $\left\|u^{-1} \mathrm{~d} u\right\|_{2^{k} p}$, which we showed above is finite. Thus, $u \in \mathcal{G}^{k+1, p}(P)$.

That is, bounds on the connection forms give bounds on the gauge transformations relating them.

\section{Non-smooth Yang-Mills connections}

In the smooth case, we defined the Yang-Mills functional (1.16), showed that critical points satisfied the weak Yang-Mills equation and said that smooth solutions to the weak equation also satisfy the strong Yang-Mills equation with boundary condition. It is possible to extend the functional to connections with less regularity as we have been discussing for the past section. For $\mathcal{Y} \mathcal{M}$ to be well defined, we need $F_{A}$ of class $L^{2}$. If $A \in \mathcal{A}^{1, p}(P)$, observing the formula for the curvature

$$
F_{A}=\mathrm{d} A+\frac{1}{2}[A \wedge A],
$$

we see that $\mathrm{d} A \in L^{p}(P, \mathfrak{g})$, so we need $p \geq 2$, and $\|[A \wedge A]\|_{2} \leq\|A\|_{4}^{2}$, hence we need the Sobolev embedding $W^{1, p} \hookrightarrow L^{4}$, which gives the condition $p \geq \frac{4 n}{4+n}$.

Definition 1.35. For $1 \leq p<\infty$ such that $p>\frac{n}{2}$, and in case $n=2$ assume in addition $p \geq \frac{4}{3}$, a connection $A \in \mathcal{A}^{1, p}(P)$ is called weak Yang-Mills if it satisfies

$$
\int_{M}\left\langle F_{A}, \mathrm{~d}_{A} \beta\right\rangle=0, \quad \forall \beta \in \Omega^{1}(M, \operatorname{ad}(P)) .
$$


This is, in fact, even weaker than the functional itself, as the functional might not be defined or finite for a weak Yang-Mills connection: we do not explicitly ask for $p \geq 2$, and while $p \geq \frac{n}{4}$ guarantees this for $n \geq 4$, this might fail if $n \leq 3$; moreover, the condition $p \geq \frac{4 n}{4+n}$ fails for $n=3$. What the conditions in the definition guarantee is that the weak equation is well defined, $\left\langle F_{A}, \mathrm{~d}_{A} \beta\right\rangle \in L^{1}(M)$. With $p \geq \frac{n}{2}$, the embedding $W^{1, p} \hookrightarrow L^{2 p}$ guarantees that $[A \wedge A]$ is in $L^{p}$, and therefore so is the curvature. Then we need $\mathrm{d}_{A} \beta$ of class $L^{p *}$, for

$$
\frac{1}{p *}:=1-\frac{1}{p}
$$

Since $\beta$ is smooth, we need $W^{1, p} \hookrightarrow L^{p *}$ for $A \in \mathcal{A}^{1, p}$, and the condition for this is $p \geq \frac{2 n}{n+1}$; for $n=1$ this is met due to $p \geq 1$, for $n \geq 3$ this is met due to $p \geq \frac{n}{2} \geq \frac{2 n}{n+1}$, and then for $n=2$ we need to assume $p \geq \frac{4}{3}$.

The following lemma shows that 1.30 is preserved under gauge transformations.

Lemma 1.36. Let $A \in A^{1, p}(P)$ be a weak Yang-Mills connection, and fix a compact set $K \subseteq M$. Then for every gauge transformation $u \in \mathcal{G}^{2, p}\left(\left.P\right|_{K}\right), u^{*} A \in \mathcal{A}^{1, p}\left(\left.P\right|_{K}\right)$ is also weak Yang-Mills.

Proof. First, we show that 1.30 still holds if we the test forms are not smooth but instead $\beta \in W^{2, p}(M, \operatorname{ad}(P))$. Since $F_{A} \in L^{p}$, we check that $\mathrm{d}_{A} \beta \in L^{p *}$. From the local formula

$$
\left(\mathrm{d}_{A} \beta\right)_{\alpha}=\mathrm{d} \beta_{\alpha}+\left[A_{\alpha} \wedge \beta_{\alpha}\right],
$$

the first term is in $L^{p *}$ since $\mathrm{d} \beta_{\alpha} \in W^{1, p}$ and $W^{1, p} \hookrightarrow L^{p *}$, and then

$$
\left\|\left[A_{\alpha} \wedge \beta_{\alpha}\right]\right\|_{p *} \leq c\left\|\left|A_{\alpha}\right|\left|\beta_{\alpha}\right|\right\|_{p *} \leq c\left\|A_{\alpha}\right\|_{p *}\left\|\beta_{\alpha}\right\|_{\infty} \leq C_{W}\left\|A_{\alpha}\right\|_{1, p}\left\|\beta_{\alpha}\right\|_{2, p},
$$

where $W^{2, p} \hookrightarrow C^{0}$ holds for $p>\frac{n}{2}$.

Now, let $K \subseteq M$ be compact and let $u \in \mathcal{G}^{2, p}\left(\left.P\right|_{K}\right)$. For a smooth test 1-form $\beta$ with support in $K$, we define $\tilde{\beta}:=u \beta u^{-1}$ and extend it to 0 outside $K$, such that $\tilde{\beta} \in$ $W^{2, p}\left(K, \operatorname{ad}\left(\left.P\right|_{K}\right)\right){ }^{11}$ We have shown that 1.30 holds for such $\tilde{\beta}$, and thus

$$
\int_{M}\left\langle F_{u^{*} A}, \mathrm{~d}_{u^{*} A} \beta\right\rangle=\int_{M}\left\langle u^{-1} F_{A} u, u^{-1} \mathrm{~d}_{A} \tilde{\beta} u\right\rangle=\int_{M}\left\langle F_{A}, \mathrm{~d}_{A} \tilde{\beta}\right\rangle=0 .
$$

This follows from the calculation below, where we use (1.23),

$$
\begin{aligned}
\left(\mathrm{d}_{A}\left(u \beta u^{-1}\right)\right)_{\alpha} & =\mathrm{d}\left(\operatorname{Ad}_{u_{\alpha}} \beta_{\alpha}\right)+\left[A_{\alpha} \wedge u_{\alpha} \beta_{\alpha} u_{\alpha}^{-1}\right] \\
& =\left[\mathrm{d} u_{\alpha} \cdot u_{\alpha}^{-1} \wedge u_{\alpha} \beta_{\alpha} u_{\alpha}^{-1}\right]+\operatorname{Ad}_{u_{\alpha}} \mathrm{d} \beta_{\alpha}+u_{\alpha}^{-1}\left[u_{\alpha} A_{\alpha} u_{\alpha}^{-1} \wedge \beta_{\alpha}\right] u_{\alpha} \\
& =u_{\alpha} \mathrm{d} \beta_{\alpha} u_{\alpha}^{-1}+u_{\alpha}\left[u_{\alpha}^{-1} \mathrm{~d} u_{\alpha} \wedge \beta_{\alpha}\right] u_{\alpha}^{-1}+u_{\alpha}^{-1}\left[u_{\alpha} A_{\alpha} u_{\alpha}^{-1} \wedge \beta_{\alpha}\right] u_{\alpha} \\
& =\left(u\left(\mathrm{~d}_{u^{*} A} \beta\right) u^{-1}\right)_{\alpha} .
\end{aligned}
$$

Next, we show that for sufficient regularity, the weak and strong Yang-Mills equations are equivalent, and this implies the result in the smooth case.

\footnotetext{
${ }^{11}$ Indeed, see lemmata 1.30 and 1.32
} 
Lemma 1.37. Let $1 \leq p<\infty$ be such that $p \geq \frac{2 n}{n+2}$ and let $k \in \mathbb{N}$. Fix a connection $A \in \mathcal{A}^{1, p}(P)$, and two equivariant forms $\omega \in W^{1, p}\left(M, \Lambda^{k} T^{*} M \otimes \operatorname{ad}(P)\right)$ and $\gamma \in L^{p}\left(M, \Lambda^{k-1} T^{*} M \otimes \operatorname{ad}(P)\right)$. Then the following are equivalent:

(i) For all smooth $\eta \in \Omega^{1}(M, \operatorname{ad}(P))$,

$$
\int_{M}\left\langle\omega, \mathrm{d}_{A} \eta\right\rangle=\int_{M}\langle\gamma, \eta\rangle
$$

(ii)

$$
\left\{\begin{array}{c}
\mathrm{d}_{A}^{*} \omega=\gamma, \\
\left.* \omega\right|_{\partial M}=0 .
\end{array}\right.
$$

Proof. Consider the calculation below,

$$
\begin{aligned}
\int_{M}\left\langle\omega, \mathrm{d}_{A} \eta\right\rangle & =\int_{M}\langle\omega, \mathrm{d} \eta\rangle+\int_{M}\langle\omega,[A \wedge \eta]\rangle \\
& =\int_{M}\left\langle\eta, \mathrm{d}^{*} \omega\right\rangle+\int_{M} \mathrm{~d}\langle\eta \wedge * \omega\rangle \pm \int_{M}\langle * \omega \wedge[A \wedge \eta]\rangle \\
& =\int_{M}\left\langle\mathrm{~d}^{*} \omega, \eta\right\rangle+\int_{\partial M}\langle\eta \wedge * \omega\rangle \pm \int_{M}\langle[\omega \omega \wedge A \wedge \eta\rangle \\
& =\int_{M}\left\langle\mathrm{~d}^{*} \omega, \eta\right\rangle-(-1)^{(n-k)(k-1)} \int_{M}\langle *[A \wedge * \omega], \eta\rangle+\int_{\partial M}\langle\eta \wedge * \omega\rangle \\
& =\int_{M}\left\langle\mathrm{~d}_{A}^{*} \omega, \eta\right\rangle+\int_{\partial M}\langle\eta \wedge * \omega\rangle
\end{aligned}
$$

where we have used Stokes's theorem and property 1.20 of the inner product on $\mathfrak{g}$-valued forms. We did not keep track of the sign in front of the $\int\langle\omega,[A \wedge \eta]\rangle$ term when writing down the calculation because it would become too cumbersome, but it can be checked that we obtain the correct sign. Moreover, the hypothesis $p \geq \frac{2 n}{n+2}$ guarantees $W^{1, p} \hookrightarrow L^{2}$, which is needed for the integral of $\langle * \omega \wedge[A \wedge \eta]\rangle$ to be well defined.

We use this identity to prove the lemma. If we assume $(i i)$, then it directly gives $(i)$. Now assume $(i)$ : testing with arbitrary $\eta$ that vanish at the boundary we get $\mathrm{d}_{A}^{*} \omega=\gamma$, and this further implies that the boundary term is zero for all $\eta$, which proves $\left.* \omega\right|_{\partial M}=0$.

The weak Yang-Mills equation is well behaved under limits, weak and strong.

Lemma 1.38. Let $1<p<\infty$ such that $p>\frac{n}{2}$, and in case $n=2$ let also $p \geq \frac{4}{3}$.

(i) If a sequence of weak Yang-Mills connections in $\mathcal{A}^{1, p}(P)$ converges strongly in the $W^{1, p}$ topology, the limit is also weak Yang-Mills.

(ii) If in case $n=2$ there is strict inequality $p>\frac{4}{3}$, for a sequence of weak Yang-Mills connections in $\mathcal{A}^{1, p}(P)$ with $L^{p}$-bound on curvature which converges weakly in the $W^{1, p}$ topology, the limit connection is also weak Yang-Mills.

Proof. We will prove $(i)$ and (ii) at the same time, commenting where the extra assumptions for $(i i)$ are needed $\sqrt{12}$ Remember that strong convergence implies weak convergence.

\footnotetext{
${ }^{12}$ Some steps would be more straightforward if we only wished to prove the case of strong convergence, however we do not comment on those.
} 
Suppose $\left(A^{i}\right) \subseteq \mathcal{A}^{1, p}(P)$ is a sequence which converges weakly to $A \in \mathcal{A}^{1, p}(P)$ such that each $A^{i}$ is weak Yang-Mills. Note that if the convergence is strong, then there is a uniform bound on $\left\|F_{A^{i}}\right\|_{p}$ a priori; if the convergence is weak, we must assume the uniform bound. Then, using the Cauchy-Schwartz ineqality and Hölder's inequality for $1=\frac{1}{p}+\frac{1}{p *}$, we prove that $A$ is also weak Yang-Mills, calculating for any $\beta \in \Omega^{1}(M, \operatorname{ad}(P))$ :

$$
\begin{aligned}
\int_{M}\left\langle F_{A}, \mathrm{~d}_{A} \beta\right\rangle & =\int_{M}\left\langle F_{A}, \mathrm{~d}_{A} \beta\right\rangle-\int_{M}\left\langle F_{A^{i}}, \mathrm{~d}_{A^{i}} \beta\right\rangle \\
& =\int_{M}\left\langle F_{A}-F_{A^{i}}, \mathrm{~d}_{A} \beta\right\rangle+\int_{M}\left\langle F_{A^{i}}, \mathrm{~d}_{A} \beta-\mathrm{d}_{A^{i}} \beta\right\rangle \\
& \leq \int_{M}\left\langle F_{A}-F_{A^{i}}, \mathrm{~d}_{A} \beta\right\rangle+\int_{M}\left|\left\langle F_{A^{i}}, \mathrm{~d}_{A} \beta-\mathrm{d}_{A^{i}} \beta\right\rangle\right| \\
& \leq \int_{M}\left\langle F_{A}-F_{A^{i}}, \mathrm{~d}_{A} \beta\right\rangle+\int_{M}\left|F_{A^{i}}\right| \cdot\left|\mathrm{d}_{A} \beta-\mathrm{d}_{A^{i}} \beta\right| \\
& \leq \underbrace{\int_{M}\left\langle F_{A}-F_{A^{i}}, \mathrm{~d}_{A} \beta\right\rangle}_{\longrightarrow 0}+\underbrace{\left\|F_{A^{i}}\right\|_{p}}_{\text {bounded }} \underbrace{\left\|\mathrm{d}_{A} \beta-\mathrm{d}_{A^{i}} \beta\right\|_{p^{*}}}_{\longrightarrow 0} \longrightarrow 0 .
\end{aligned}
$$

The first limit is the weak $L^{p}$-convergence of $F_{A^{i}}$, and we check weak convergence of local representatives on all bundle charts $U_{\alpha},\left(F_{A^{i}}\right)_{\alpha}=\mathrm{d} A_{\alpha}^{i}+\left[A_{\alpha}^{i} \wedge A_{\alpha}^{i}\right]$. For the weak convergence of the $\mathrm{d} A_{\alpha}^{i}$ term, test with any $\beta \in \Omega^{2}\left(U_{\alpha}, \mathfrak{g}\right)$ that vanishes on $\partial U_{\alpha}$,

$$
\int_{U_{\alpha}}\left\langle\mathrm{d} A_{\alpha}^{i}, \beta\right\rangle=\int_{U_{\alpha}}\left\langle A_{\alpha}^{i}, \mathrm{~d}^{*} \beta\right\rangle \stackrel{i \rightarrow \infty}{\longrightarrow} \int_{U_{\alpha}}\left\langle A_{\alpha}, \mathrm{d}^{*} \beta\right\rangle=\int_{U_{\alpha}}\left\langle\mathrm{d} A_{\alpha}, \beta\right\rangle
$$

then note that the limit holds for all $\beta \in \Omega^{2}\left(U_{\alpha}, \mathfrak{g}\right)$ since these can be $L^{p *}$-approximated by such test forms which vanish on the boundary ${ }^{13}$ For the $\left[A_{\alpha}^{i} \wedge A_{\alpha}^{i}\right]$ term, use Hölder's inequality for $\frac{1}{p}=\frac{1}{2 p}+\frac{1}{2 p}$,

$$
\left\|\left[A_{\alpha} \wedge A_{\alpha}\right]-\left[A_{\alpha}^{i} \wedge A_{\alpha}^{i}\right]\right\|_{p} \leq\left\|A_{\alpha}-A_{\alpha}^{i}\right\|_{2 p}\left\|A_{\alpha}\right\|_{2 p}+\left\|A_{\alpha}^{i}\right\|_{2 p}\left\|A_{\alpha}-A_{\alpha}^{i}\right\|_{2 p} \longrightarrow 0,
$$

and this converges strongly because of the compact Sobolev embedding $W^{1, p} \hookrightarrow L^{2 p}$ guaranteed by $p>\frac{n}{2}$.

As for the second limit, we again look at local representatives,

$$
\left(\mathrm{d}_{A} \beta-\mathrm{d}_{A^{i}} \beta\right)_{\alpha}=\left[\left(A_{\alpha}-A_{\alpha}^{i}\right) \wedge \beta_{\alpha}\right],
$$

and obtain convergence in all bundle charts observing the following:

$$
\left\|\left(\mathrm{d}_{A} \beta-\mathrm{d}_{A^{i}} \beta\right)_{\alpha}\right\|_{p *} \leq\left\|A_{\alpha}-A_{\alpha}^{i}\right\|_{p *} \cdot\left\|\beta_{\alpha}\right\|_{\infty} \longrightarrow 0 .
$$

This limit follows from the continuous embedding $W^{1, p} \hookrightarrow L^{p *}$ if the original sequence converged strongly in $\mathcal{A}^{1, p}$, however if we only had weak convergence the additional hypothesis $p>\frac{4}{3}$ is needed to ensure that the embedding is compact and therefore there is strong convergence in $L^{p *}$.

\footnotetext{
${ }^{13}$ Writing $\beta_{i}$ for the test forms vanishing on $\partial U_{\alpha}$ approximating $\beta$, using Hölder's inequality and noting that the compact embedding $W^{1, p} \hookrightarrow L^{p}$ gives a uniform bound on $\left\|A_{\alpha}^{i}\right\|_{p}$, the boundary term coming from Stokes's theorem will be bounded by $\left\|A_{\alpha}^{i}\right\|_{p}\left\|\beta-\beta_{i}\right\|_{p *} \rightarrow 0$.
} 
Finally, we state without proof the main result we will need on the regularity of YangMills connections.

Proposition 1.39. Let $M$ be a compact n-manifold, and let $\tilde{A} \in \mathcal{A}(P)$ be a smooth reference connection. Let $1<p<\infty$ and $k \in \mathbb{N}$ be such that either $k p>n$, or if $k=1$ then $\frac{n}{2}<p<n$, and in either case if $n=2$, then $p \geq \frac{4}{3}$. Moreover, let $q:=p$ in the first case or $q:=\frac{n p}{2 n-p}$ in the second case. Then there exists a constant $C$ with the following significance:

Let $A=\tilde{A}+\alpha \in \mathcal{A}^{k, p}(P)$ be a connection that satisfies

$$
\left\{\begin{array}{c}
\mathrm{d}_{\tilde{A}}^{*} \alpha=0 \\
\left.* \alpha\right|_{\partial M}=0
\end{array}\right.
$$

and for all smooth $\beta \in \Omega^{1}(M, \operatorname{ad}(P))$

$$
\int_{M}\left\langle F_{A}, \mathrm{~d}_{A} \beta\right\rangle=0
$$

Then $A \in \mathcal{A}^{k+1, q}(P)$ and

$$
\|\alpha\|_{k+1, q} \leq C\left(1+\|\alpha\|_{k, p}+\|\alpha\|_{k, p}^{3}\right) .
$$

Moreover, the constant $C$ can be chosen such that it depends $W^{k+1, \infty}$-continuously on the metric.

Proof. See [Weh04, corollary 9.6.

The first pair of equations in this proposition may seem arbitrary, but we will meet them again in the next chapter. This result essentially proves the regularity of the Yang-Mills operator in Coulomb gauge. 


\section{Chapter 2}

\section{The GAUGE FIXING LEMMA}

The main theorem in this chapter is very easy to state in simple terms: there always exists a good gauge locally. In the first section we motivate the idea behind gauge fixing and show an example of a good gauge in the simple case of $G$ abelian and $M$ a closed manifold, before giving the main definition 2.1 of what a "good" gauge is in the present text, and explaining how the main theorem 2.2 follows from a similar result in Euclidean space. Then, in the second section, we prove the result on a chart.

The gauge fixing lemma will be essential for the proofs of the compactness theorems in the next chapter.

\subsection{Motivation and main result}

\section{Flat connections, Coulomb gauge and Hodge theory}

In a problem that is gauge invariant, solutions come in families (equivalence classes) and one may wish to choose representatives for some reason. For instance, it may simplify calculations, as in the choice of gauge $\nabla \cdot A=0$ for the vector potential in electromagnetism; it may minimize some norm, or choose some specific representative that has some other important meaning.

If a connection is flat, then we know that we may choose a trivialization such that the connection matrix is identically zero, that is, $\nabla^{A}=\mathrm{d}$ is the canonical or product connection on a trivial vector bundle. This may be done with parallel transport, by first choosing a frame over a point $p$ and then extending this frame in each direction on the manifold. Similarly, on a holomorphic vector bundle one may wish to choose trivializations such that the partial connection $\bar{\partial}_{\alpha}=\bar{\partial}+\alpha$ has $\alpha=0$, that is, choose a gauge such that the flat-in-the- $(0,1)$-direction connection is the canonical one 1

So if a connection is somehow flat, one obtains local gauges such that the connection matrix is zero. If instead of flatness we have small curvature, can one find a correspondingly small connection matrix? In the case of the abelian $U(1)$ gauge group ${ }^{2}$ we may use Hodge theory. Assume $M$ is a simply connected closed base manifold for the time being, and let $A$ be a connection on a trivial $U(1)$-bundle over $M$; recall that the Lie algebra of $U(1)$ is $i \mathbb{R}$. The curvature of the connection is simply $F_{A}=\mathrm{d} A$, and any change $A \mapsto A+i \mathrm{~d} f$ for a smooth real valued function leaves the curvature unchanged. If $u: M \rightarrow U(1)$ is a gauge

\footnotetext{
${ }^{1}$ For the detailed statements and proofs of these results, see [DK97], section 2.2

${ }^{2}$ This corresponds to classical electromagnetism, see appendix ??.
} 
transformation, then it may be written as $u(x)=\exp ($ if $(x))$ and

$$
u^{-1} \mathrm{~d} u=\exp (-i f) \mathrm{d} \exp (i f)=i \mathrm{~d} f,
$$

so that indeed $A \mapsto A+i \mathrm{~d} f$ is a gauge transformation. We want to minimize the $L^{2}$-norm of the connection

$$
\int_{M}|A|^{2}
$$

along this gauge equivalent family. The Euler-Lagrange equation for this functional is

$$
\mathrm{d}^{*} A=0 .
$$

Indeed,

$$
\begin{aligned}
\left.\frac{\mathrm{d}}{\mathrm{d} t}\right|_{t=0}\langle A+i t \mathrm{~d} f, A+i t \mathrm{~d} f\rangle & =2 i\langle\mathrm{~d} f, A\rangle \\
& =2 i\left\langle f, \mathrm{~d}^{*} A\right\rangle=0, \forall f \in C^{\infty}(M)
\end{aligned}
$$

implies that $\mathrm{d}^{*} A=0$. Thus we wish to find $f$ such that $\tilde{A}:=A+i \mathrm{~d} f$ is gauge equivalent to $A$, and

$$
\mathrm{d}^{*} \tilde{A}=\mathrm{d}^{*}(A+i \mathrm{~d} f)=0 .
$$

This is equivalent to

$$
\Delta f=-i \mathrm{~d}^{*} A .
$$

From the Hodge decomposition theorem 3 we know that there is a solution $f$ if and only if $\mathrm{d}^{*} A$ is orthogonal to the kernel of the Laplacian, which in this case consists of the constant functions. So what we want is that $\int-1 \cdot i \mathrm{~d}^{*} A=0$, or $\int \mathrm{d}^{*} A=0$. But

$$
\int_{M} \mathrm{~d}^{*} A= \pm \int_{M} * \mathrm{~d} * A * 1= \pm \int_{M} \mathrm{~d} * A=0
$$

by Stokes's theorem, since we are assuming that $M$ has no boundary. Therefore we find a solution $f$. The proof that this critical point of the functional is in fact a minimum is a little more involved and we skip it, as there seems to be no insight to be gained from it in this context. More interesting than that is the fact that $d+d^{*}$ is an elliptic operator, which provides the following estimate for some constant $C$ (since $M$ is simply connected and so $\left.H^{1}(M)=0\right)^{4}$

$$
\|A\|_{k, p} \leq C\left(\|\mathrm{~d} A\|_{k-1, p}+\left\|\mathrm{d}^{*} A\right\|_{k-1, p}\right) .
$$

When the gauge is fixed such that $\mathrm{d}^{*} A=0$, and in the abelian case with $F_{A}=\mathrm{d} A$, we then have

$$
\|A\|_{k, p} \leq C\left\|F_{A}\right\|_{k-1, p}
$$

and so we showed that there exists an optimal gauge choice such that it minimizes the norm of the connection matrix, and moreover this norm is bounded by the norm of the curvature, such that small curvature leads to small connection, as we wished. As a sanity check, note that if the connection is flat, then the Hodge gauge indeed forces the connection matrix to be zero.

\footnotetext{
${ }^{3}$ See e.g. War83], theorem 6.8, or [DK97], theorem (A.7), which they call "the Fredholm alternative".

${ }^{4}$ Alternatively, see theorem 1.26
} 


\section{Uhlenbeck's gauge fixing lemma}

In the discussion above we considered the case of an abelian gauge group action on a trivial bundle over a simply connected compact manifold without boundary. Each of these hypotheses were important for this straightforward development: the vanishing bracket let us write the linear equation $F_{A}=\mathrm{d} A$; the triviality of the bundle let us work with the local representation of connections and gauge transformations; $H^{1}(M)=0$ and compactness were needed for the elliptic estimate, and the empty boundary hypothesis was used for Stokes's theorem and to avoid dealing with (elliptic) boundary conditions. What we want now is for a similar result to hold locally on any smooth manifold with a non-abelian gauge group action on a bundle that is not necessarily trivial. Of course, if the result is local we may choose trivializing neighbourhoods and essentially work on a trivial bundle over the unit ball on Euclidean space. For elliptic theory to hold, we need the closed ball for compactness, but now the boundary is not empty and we will need a suitable boundary condition. Finally, for a non-abelian gauge group, the curvature is $F_{A}=\mathrm{d} A+\frac{1}{2}[A \wedge A]$ which leads to non-linear equations. We assert that the gauge we used above, supplemented with a suitable boundary condition, is still an interesting and profitable gauge choice. Indeed, on the closed ball, the Euler-Lagrange equations for the $\int_{B}|A|^{2}$ functional are 5

$$
\left\{\begin{aligned}
\mathrm{d}^{*} A & =0, \\
\left.* A\right|_{\partial U} & =0 .
\end{aligned}\right.
$$

Issues can arise when directly minimizing the norm this way, as the gauges constructed can have singularities; however, in the small curvature regime this is not a problem 6 As we shall see later, these equations also fit in nicely with the Yang-Mills equation and yield an elliptic system. Finally, we would once again like for bounds on curvature to translate into bounds on the connection matrix. With all of these considerations, the following definition should feel natural:

Definition 2.1 (Uhlenbeck gauge). Let $(M, g)$ be a Riemannian manifold, let $G$ be a compact Lie group, and let $P \rightarrow M$ be a principal $G$-bundle.

Let $U \subseteq M$ be a trivializing neighbourhood of $P$. We say that a connection $A \in \mathcal{A}^{1, p}(U)$ is in Uhlenbeck gauge with constant $\tilde{C}$ if it satisfies

$$
\left\{\begin{aligned}
\mathrm{d}^{*} A=0 & \text { on } U \\
\left.* A\right|_{\partial U}=0 & \text { on } \partial U
\end{aligned}\right.
$$

and

$$
\|A\|_{1, s} \leq \tilde{C}\left\|F_{A}\right\|_{s}
$$

for $s=p$ or $q, q$ as in the following theorem.

Our main theorem is then on the local existence of Uhlenbeck gauges.

Theorem 2.2 (Gauge fixing). Suppose that $1<q \leq p<\infty$ such that $q \geq \frac{n}{2}, p>\frac{n}{2}$, and in case $q<n, p \leq \frac{n q}{n-q}$. Then there exist constants $\tilde{C}$ and $\tilde{\varepsilon}>0$ such that the following holds:

For every point in $M$, there is a neighbourhood $U \subseteq M$ such that for every connection $A \in \mathcal{A}^{1, p}(U)$ with $\mathcal{E}_{q}(A) \leq \tilde{\varepsilon}$ there exists a gauge transformation $u \in \mathcal{G}^{2, p}(U)$ such that $\tilde{A}:=u^{*} A$ is in Uhlenbeck gauge. Note that $\left|F_{\tilde{A}}\right|=\left|F_{A}\right|$.

\footnotetext{
${ }^{5}$ See lemma 1.37 .

${ }^{6}$ See [FU91], the comment right before Lemma 8.2 on page 119
} 
Originally, this theorem was proved by Uhlenbeck in 1982 for $n>p \geq \frac{n}{2}$ and $q=\frac{n}{2}$ on the unit ball; it is theorem 2.1 in Uhl82. The condition $p>\frac{n}{2}$ guarantees that the gauge group is indeed a topological group with continuous action. Nevertheless, it is possible to extend the result for $p=\frac{n}{2}$ by a weak-limit argument.

Corollary 2.3. The theorem also holds for $p=q=\frac{n}{2}$ if $n \geq 3$.

Proof. See [Weh04], proof of remark 6.2a), page 105.

It suffices to prove the theorem on a coordinate chart, since the result is local and the Uhlenbeck gauge conditions are invariant under change of coordinates on the base manifold. Thus, we now state the theorem on an open set in Euclidean (half) space, which we will prove in the next section, and then show how this implies theorem 2.2 .

Proposition 2.4. Let $G$ be a compact Lie group and $B \subseteq \mathbb{R}^{n}$ the open unit ball or the "egg"7. Suppose that $1<q \leq p<\infty$ such that $q \geq \frac{n}{2}, p>\frac{n}{2}$, and in case $q<n, p \leq \frac{n q}{n-q}$. Then there exist constants $\tilde{C}, \tilde{\varepsilon}>0$ and $\delta>0$ such that the following holds:

If $B$ is equipped with a smooth metric $g$ such that $\|g-\mathbb{1}\|_{2, \infty} \leq \delta$ then for every connection $A \in \mathcal{A}^{1, p}(B)$ with $\mathcal{E}(A) \leq \tilde{\varepsilon}$ there exists a gauge transformation $u \in \mathcal{G}^{2, p}(B)$ such that $u^{*} A$ is in Uhlenbeck gauge with respect to the metric $g$ and with constant $\tilde{C}$.

Proof of Theorem 2.2. Take $\delta>0$ from Proposition 2.4 and take $B$ as follows:

- For $p$ in the interior of $M, B \subseteq \mathbb{R}^{n}$ is the unit ball around the origin.

- For $p \in \partial M$, the "egg" $B$ is an open subset of the half space $\mathbb{H}^{n}=\left\{\left(x_{1}, \ldots, x_{n}\right) \in\right.$ $\left.\mathbb{R}^{n}: x_{1} \geq 0\right\}$ that contains a neighbourhood of 0 in $\partial \mathbb{H}^{n}$, is starshaped relative to 0 and has smooth boundary 8

For $p \in M$, choose a coordinate chart around $p, \psi: V \rightarrow M$ for $V \subseteq \mathbb{R}^{n}$ or $\mathbb{H}^{n}$, such that $\psi^{*} g(0)=\mathbb{1} .^{9}$ For some small $\sigma \in(0,1], \sigma B \subseteq V$ and we can restrict $\psi: \sigma B \rightarrow M$. To get a chart on $B$, consider $\psi_{\sigma}:=\psi \circ \sigma: B \rightarrow M$. The pullback metric is $\psi_{\sigma}^{*} g(z)=\sigma^{2} \psi^{*} g(\sigma z)$; indeed,

$$
\begin{aligned}
\psi_{\sigma}^{*} g(v, w)(z) & =(\psi \circ \sigma)^{*} g(v, w)(z) \\
& =\psi^{*} g\left(\mathrm{~d}_{z} \sigma v, \mathrm{~d}_{z} \sigma w\right)(\sigma z) \\
& =\sigma^{2} \psi^{*} g(v, w)(\sigma z) .
\end{aligned}
$$

Note that $\psi_{\sigma}^{*} g(0)=\sigma^{2} \mathbb{1}$, and so this metric is not close to the identity, but if we simply rescale by $\sigma^{-2}$, then $\sigma^{-2} \psi_{\sigma}^{*} g(z)=\psi^{*} g(\sigma z)$ is $W^{2, \infty}$-close to the identity, as the first derivative is $\nabla\left(\psi^{*} g \circ \sigma\right)(z)=\sigma \nabla\left(\psi^{*} g\right)(\sigma z)$, and the second derivative is

$$
\begin{aligned}
\nabla^{2}\left(\sigma^{-2} \psi_{\sigma}^{*} g\right)(z) & =\nabla^{2}\left(\psi^{*} g \circ \sigma\right)(z) \\
& =\nabla\left(\sigma\left(\nabla \psi^{*} g \circ \sigma\right)\right)(z) \\
& =\sigma^{2} \nabla^{2} \psi^{*} g(\sigma z) .
\end{aligned}
$$

\footnotetext{
${ }^{7}$ See definition below.

${ }^{8}$ This type of domain is called an "egg squeezed to the boundary" in Weh04.

${ }^{9}$ Note that this is always possible, as you can simply choose an orthonormal basis on $T_{p} M$ and then parallel transport it to get a local frame over a contractible domain.
} 
Then, because $\psi^{*} g$ is smooth on the closure of $\sigma B$ (which is compact), these derivatives are bounded and can be made small by the choice of $\sigma$.

Now, having chosen $\sigma$ such that $\left\|\sigma^{-2} \psi_{\sigma}^{*} g-\mathbb{1}\right\|_{2, \infty} \leq \delta$, Proposition 2.4 holds on $B$ with metric $g_{B}:=\sigma^{-2} \psi_{\sigma}^{*} g$. However, in order to obtain the result on $U:=\psi(\sigma B) \subseteq M$ with the intended metric $g$, we need to show that the result still holds on $B$ with metric $\sigma^{2} g_{B}=\psi_{\sigma}^{*} g$.

On the effect of the conformal change of metric on $\mathcal{A}, \mathcal{G}$ and condition (2.1), note that over a compact manifold the metrics are equivalent and so the spaces are the same, and as for the equations, the change of metric affects the Hodge star, however only by possible conformal scalings, and the equations still hold.

What is left is to check that the bounds 2.2 are still valid with the same constant $\tilde{C}$. First, we look at the effect of the rescaling on the norm of the curvature:

$$
\begin{aligned}
\left\|F_{A}\right\|_{\sigma^{2} g ; q}^{q} & =\int_{B}\left|F_{A}\right|_{\sigma^{2} g}^{q} \sqrt{\operatorname{det}\left(\sigma^{2} g\right)} \mathrm{d}^{n} x \\
& =\int_{B}\left(\sigma^{-2} g^{i k} \sigma^{-2} g^{j l}\left(F_{A}\right)_{i j}\left(F_{A}\right)_{k l}\right)^{\frac{q}{2}} \sqrt{\sigma^{2 n} \operatorname{det}(g)} \mathrm{d}^{n} x \\
& =\sigma^{n-2 q}\left\|F_{A}\right\|_{g ; q}^{q} .
\end{aligned}
$$

For $q$ as in the theorem, this is the $L^{q}$-energy $\mathcal{E}_{\sigma^{2} g ; q}(A)$. If it is bounded $\mathcal{E}_{\sigma^{2} q ; q}(A) \leq \tilde{\varepsilon}$, then since $q \geq \frac{n}{2}$ and $\sigma \leq 1, \mathcal{E}_{g ; q}(A) \leq \sigma^{2 q-n} \tilde{\varepsilon}$ gives $\mathcal{E}_{g ; q}(A) \leq \tilde{\varepsilon}$. The calculation for the $L^{p}$-norm is the same. For the $W^{1, p}$-norm of a connection $A \in \mathcal{A}^{1, p}(B)$, we first have the straightforward calculation

$$
\|A\|_{\sigma^{2} g ; p}^{p}=\int_{B}\left(\sigma^{-2} g^{i j} A_{i} A_{j}\right)^{\frac{p}{2}} \sqrt{\operatorname{det}\left(\sigma^{2} g\right)} \mathrm{d}^{n} x=\sigma^{n-p}\|A\|_{g ; p}^{p},
$$

and then for the covariant derivative note that $(\nabla A)_{i j}=\nabla_{i} A_{j}-\Gamma_{i j}^{k} A_{k}$ and the Christoffel symbols for $\sigma^{2} g$ and $g$ are the same 10 Then $\nabla^{g} A=\nabla^{\sigma^{2} g} A$ and as for the curvature

$$
\left\|\nabla^{\sigma^{2} g} A\right\|_{\sigma^{2} g ; p}^{p}=\sigma^{n-2 p}\left\|\nabla^{g} A\right\|_{g ; p}^{p} .
$$

Putting both terms together we have

$$
\|A\|_{\sigma^{2} g ; 1, p}^{p}=\sigma^{n-p}\|A\|_{g ; 1, p}^{p}+\sigma^{n-2 p}\|\nabla A\|_{g ; 1, p}^{p} \leq \sigma^{n-2 p}\|A\|_{g ; 1, p}^{p} .
$$

Finally, if $A$ is a connection that has been put in Uhlenbeck gauge and satisfies (2.2) with respect to the metric $\sigma^{2} g$, then simply concatenating our inequalities

$$
\|A\|_{\sigma^{2} g ; 1, p} \leq \sigma^{\frac{n-2 p}{p}}\|A\|_{g ; 1, p} \leq \tilde{C} \sigma^{\frac{n-2 p}{p}}\left\|F_{A}\right\|_{g ; p}=\tilde{C}\left\|F_{A}\right\|_{\sigma^{2} g ; p}
$$

we see that $A$ also satisfies $(2.2)$ with respect to the metric $g$ and with the same constant $\tilde{C}$.

\subsection{The big bad proof}

This proof is the main technical result of this text. It will refer to most of the lemmas from the preceding chapter and appendix A. For its relevance (and length), we give it its

\footnotetext{
${ }^{10}$ Indeed, $\Gamma_{i j}^{k}=$ a sum of multiples (components of $\left.g^{-1}\right) \times($ derivatives of components of $g$ ), and so a constant rescaling is cancelled.
} 
own section. The proof will go through via the continuous induction method, and its main interesting features are the use of the implicit function theorem, boundary value spaces, the elliptic estimate for the $\mathrm{d}+\mathrm{d}^{*}$ operator and results relating to the Neumann problem.

Define the modified energy $\mathcal{E}_{q}^{\prime}(A)=\int_{B}\left|F_{A}(x)\right|^{q} \mathrm{~d} x^{n}$ using the Euclidean metric on $B$ for a connection $A$, note that if $\delta$ is small enough in $\|g-\mathbb{1}\|_{2, \infty} \leq \delta$, then $g$ is sufficiently close to the identity that

$$
\frac{1}{2} \mathcal{E}_{q}^{\prime}(A) \leq \mathcal{E}_{q}(A) \leq 2 \mathcal{E}_{q}^{\prime}(A)
$$

Define

$$
\mathcal{A}_{\varepsilon}:=\left\{A \in \mathcal{A}^{1, p}(B): \mathcal{E}^{\prime}(A)<\varepsilon\right\}
$$

and

$$
S_{\varepsilon}:=\left\{A \in \mathcal{A}_{\varepsilon} \text { such that } A \text { can be put into Uhlenbeck gauge }\right\} .
$$

We will show that $S_{\varepsilon}=A_{\varepsilon}$, and thus every connection with energy at most $\tilde{\varepsilon}:=\frac{\varepsilon}{2}$ can be put into Uhlenbeck gauge.

The proof will have three steps, and during each step some care has to be taken with the constants $\varepsilon, \tilde{C}$ and $\delta$ and their dependence on each other and the metric on $B$ :

(1) For some fixed $g$ and $\varepsilon$, we prove $\mathcal{A}_{\varepsilon}$ is connected.

(2) For some fixed $g, \varepsilon$ and $\tilde{C}, S_{\varepsilon}$ is closed.

(3) We find $\varepsilon, \tilde{C}$ and $\delta$, and vary the metric with $\delta$, such that $S_{\varepsilon}$ is open.

Throughout, we take the local descriptions explained in subsection 1.2.1. Therefore, a connection in $\mathcal{A}^{1, p}(B)$ as defined in 1.25 is a $\mathfrak{g}$-valued 1-form on $B$ and a gauge transformation in $\mathcal{G}^{2, p}(U)$ as defined in 1.26 is a function $u: B \rightarrow G$.

\section{(1) $\mathcal{A}_{\varepsilon}$ is connected.}

Proposition 2.5. Let $\bar{B}$ be equipped with any smooth metric $g$ and let $\varepsilon>0$; then $\mathcal{A}_{\varepsilon}$ as defined above is connected.

Proof. We prove there is a continuous path from each $A \in \mathcal{A}_{\varepsilon}$ to the canonical flat connection in $\mathcal{A}_{\varepsilon}$, which is represented by $A \equiv 0 \in \Omega^{1}(B, \mathfrak{g})$.

Let $A \in \mathcal{A}_{\varepsilon}$, and define the path $\left(A_{\sigma}\right)_{\sigma \in[0,1]}$ by $A_{\sigma}(x)=\sigma^{*} A(x)=\sigma A(\sigma x)$ where we take the pullback under the map $x \mapsto \sigma x{ }^{11}$ Clearly, $A_{0}=0$ and $A_{1}=A$, and for each $\sigma \in[0,1], A_{\sigma} \in \mathcal{A}^{1, p}(B)$. The curvature of the connection $A_{\sigma}$ is 12

$$
F_{A_{\sigma}}(x)=\mathrm{d}\left(A_{\sigma}\right)+\frac{1}{2}\left[A_{\sigma} \wedge A_{\sigma}\right]=\sigma^{2} \mathrm{~d} A(\sigma x)+\frac{\sigma^{2}}{2}[A(\sigma x) \wedge A(\sigma x)]=\sigma^{2} F_{A}(\sigma x),
$$

and so we have

$$
\mathcal{E}_{q}^{\prime}\left(A_{\sigma}\right)=\int_{B} \sigma^{2 q}\left|F_{A}(\sigma x)\right|^{q} \mathrm{~d} x^{n}=\sigma^{2 q-n} \int_{\sigma B}\left|F_{A}(y)\right|^{q} \mathrm{~d} y^{n} \leq \sigma^{2 q-n} \mathcal{E}^{\prime}(A) \leq \varepsilon,
$$

where we changed variables $y=\sigma x$ and used the assumption that $q \geq \frac{n}{2}$ for $\sigma^{2 q-n} \leq 1$. Therefore, the whole path is contained in $\mathcal{A}_{\varepsilon}$.

\footnotetext{
${ }^{11}$ This is well defined since $B$ is star-shaped with respect to 0 and therefore the path $\sigma x$ stays within $B$.

${ }^{12}$ Use $\mathrm{d}_{x}\left(A_{\sigma}\right)=\mathrm{d}_{x}(\sigma \circ A \circ \sigma)$, and recall that the differential of a linear map is itself.
} 
To show the continuity of the path, we will use the Euclidean metric on $\bar{B}$ instead of the metric $g$. For continuity at $\sigma=0$, since $A_{0}=0$, it suffices to show that $\left\|A_{\sigma}\right\|_{\mathbb{1} ; 1, p} \stackrel{\sigma \rightarrow 0}{\longrightarrow} 0$. We look at the two $L^{p}$-norms separately. First, we have

$$
\begin{aligned}
\left\|A_{\sigma}\right\|_{\mathbb{1} ; p(B)}^{p} & =\int_{B}\left|A_{\sigma}\right|^{p} \mathrm{~d} x^{n} \\
& =\sigma^{p} \int_{B}|A(\sigma x)|^{p} \mathrm{~d} x^{n} \\
& =\sigma^{p-n} \int_{\sigma B}|A(y)|^{p} \mathrm{~d} y^{n} \\
& =\sigma^{p-n}\|A\|_{\mathbb{1} ; p(\sigma B)}^{p} \\
& \stackrel{(1)}{\leq} \sigma^{p-n}\|1\|_{\mathbb{1} ; 2 p(\sigma B)}^{p}\|A\|_{\mathbb{1} ; 2 p(\sigma B)}^{p} \\
& =\sigma^{p-n} \operatorname{Vol}(\sigma B)^{\frac{1}{2}}\|A\|_{\mathbb{1} ; 2 p(\sigma B)}^{p} \\
& \stackrel{(2)}{\leq} \sigma^{p-n+\frac{n}{2}} \operatorname{Vol}(B)^{\frac{1}{2}} C_{W}\|A\|_{\mathbb{1} ; 1, p(\sigma B)}^{p} \\
& \leq \underbrace{\sigma^{p-\frac{n}{2}}}_{\rightarrow 0} \underbrace{\operatorname{Vol}(B)^{\frac{1}{2}} C\|A\|_{\mathbb{1} ; 1, p(B)}^{p}}_{\text {constant }} \longrightarrow 0,
\end{aligned}
$$

where in (1) we used the Hölder inequality and (2) is the Sobolev inequality for $W^{1, p} \hookrightarrow L^{2 p}$ guaranteed by the hypothesis $p>\frac{n}{2}$, which is also used for $\sigma^{p-\frac{n}{2}} \rightarrow 0$. For the derivative term,

$$
\begin{aligned}
\left\|\nabla A_{\sigma}\right\|_{\mathbb{1} ; p(B)} & =\int_{B}\left|\nabla A_{\sigma}(x)\right|^{p} \mathrm{~d} x^{n} \\
& =\int_{B} \sigma^{p}|\nabla(A \circ \sigma)(x)|^{p} \mathrm{~d} x^{n} \\
& =\sigma^{2 p} \int_{B}|\nabla A(\sigma x)|^{p} \mathrm{~d} x^{n} \\
& =\sigma^{2 p-n} \int_{\sigma B}|\nabla A(y)|^{p} \mathrm{~d} y^{n} \\
& =\sigma^{2 p-n}\|\nabla A\|_{\mathbb{1} ; p(\sigma B)}^{p} \longrightarrow 0
\end{aligned}
$$

where we simply use a change of variables and again need the condition $p>\frac{n}{2}$ for convergence.

The continuity at $\sigma_{0} \in(0,1]$ is more convoluted, and we will make use of an auxiliary sequence $\left(A^{i}\right)_{i \in \mathbb{N}} \subseteq \mathcal{A}(B)$ of smooth connections which converge to $A$ in $\mathcal{A}^{1, p}(B)$ :

$$
\begin{aligned}
\left|A_{\sigma}(x)-A_{\sigma_{0}}(x)\right|=\mid & \left|\sigma A(\sigma x)-\sigma_{0} A\left(\sigma_{0} x\right)\right| \\
=\mid & \mid \sigma A(\sigma x)-\sigma A^{i}(\sigma x)+\sigma A^{i}(\sigma x)-\sigma A^{i}\left(\sigma_{0} x\right)+\sigma A^{i}\left(\sigma_{0} x\right) \\
& \quad-\sigma A\left(\sigma_{0} x\right)+\sigma A\left(\sigma_{0} x\right)-\sigma_{0} A\left(\sigma_{0} x\right) \mid \\
\leq & \left|A(\sigma x)-A^{i}(\sigma x)\right|+\sigma\left|A^{i}(\sigma x)-A^{i}\left(\sigma_{0} x\right)\right| \\
& +\sigma\left|A^{i}\left(\sigma_{0} x\right)-A\left(\sigma_{0} x\right)\right|+\left|\sigma-\sigma_{0}\right|\left|A\left(\sigma_{0} x\right)\right| \\
\leq & \left|A(\sigma x)-A^{i}(\sigma x)\right|+\left|\sigma-\sigma_{0}\right| C_{i} \\
& +\left|A^{i}\left(\sigma_{0} x\right)-A\left(\sigma_{0} x\right)\right|+\left|\sigma-\sigma_{0}\right|\left|A\left(\sigma_{0} x\right)\right|
\end{aligned}
$$


where we have used $\sigma \leq 1$ and the mean value inequality, proposition A.2 for $\left\|\nabla A^{i}(x)\right\| \leq$ $C_{i}$, since the $A_{i}$ and all of their derivatives are bounded on $\bar{B}$. Now we apply the Euclidean norm $\|\cdot\|_{\mathbb{1} ; p(B)}$ to this inequality ${ }^{13}$ and change variables to obtain

$$
\begin{aligned}
\left\|A_{\sigma}-A_{\sigma_{0}}\right\|_{\mathbb{1} ; p} \leq & \sigma^{-\frac{n}{p}}\left\|A-A^{i}\right\|_{\mathbb{1} ; p(\sigma B)}+\left|\sigma-\sigma_{0}\right| C_{i} \operatorname{Vol}(B)^{\frac{1}{p}} \\
& +\sigma_{0}^{-\frac{n}{p}}\left\|A-A^{i}\right\|_{\mathbb{1} ; p\left(\sigma_{0} B\right)}+\left|\sigma-\sigma_{0}\right| \sigma_{0}^{-\frac{n}{p}}\|A\|_{\mathbb{1} ; p\left(\sigma_{0} B\right)} \\
\leq & \left(\sigma^{-\frac{n}{p}}+\sigma_{0}^{-\frac{n}{p}}\right)\left\|A-A^{i}\right\|_{\mathbb{1} ; p(B)}+\left|\sigma-\sigma_{0}\right| C_{i} \operatorname{Vol}(B)^{\frac{1}{p}} \\
& +\left|\sigma-\sigma_{0}\right| \sigma_{0}^{-\frac{n}{p}}\|A\|_{\mathbb{1} ; p(B)} .
\end{aligned}
$$

It is now necessary to be a bit careful about the mixed terms depending on $i$ and $\sigma$, but for fixed $\sigma_{0}$ the right-hand side can be made as small as we want in the following way: first, take $\sigma$ close to $\sigma_{0}$ such that $\sigma^{-\frac{n}{p}}+\sigma_{0}^{-\frac{n}{p}}$ is bounded, say, by $2 \sigma_{0}^{-\frac{n}{p}}+c$ for some constant $c$; then take $i$ such that the first term is small; for this fixed $i, C_{i}$ is also constant and therefore a further suitable choice of $\sigma$ even closer to $\sigma_{0}$ makes the second and third terms small.

The computation to check that $\left\|\nabla A_{\sigma}-\nabla A_{\sigma_{0}}\right\| \stackrel{\sigma \rightarrow \sigma_{0}}{\longrightarrow} 0$ is completely analogous, using bounds $C_{i}^{\prime}$ on the second derivatives of $A^{i}$, yielding

$$
\begin{aligned}
\left\|\nabla A_{\sigma}-\nabla A_{\sigma_{0}}\right\|_{\mathbb{1} ; p(B)} \leq & \left(\sigma^{-\frac{n}{p}}+\sigma_{0}^{-\frac{n}{p}}\right)\left\|\nabla A-\nabla A^{i}\right\|_{\mathbb{1} ; p(B)}+\left|\sigma-\sigma_{0}\right| C_{i}^{\prime} V o l(B)^{\frac{1}{p}} \\
& +\left|\sigma^{2}-\sigma_{0}^{2}\right| \sigma_{0}^{-\frac{n}{p}}\|\nabla A\|_{\mathbb{1} ; p(B)} \longrightarrow 0
\end{aligned}
$$

for suitable choices of $i$ and $\sigma$ close to $\sigma_{0}$ as before.

\section{(2) $S_{\varepsilon}$ is closed}

Proposition 2.6. Let $B$ be equipped with any smooth metric $g$ and let $\varepsilon>0$. Suppose that there is a sequence $\left(A_{i}\right)_{i \in \mathbb{N}} \subseteq \mathcal{A}_{\varepsilon}$ converging to some $A \in \mathcal{A}_{\varepsilon}$ such that each $A_{i}$ can be put into Uhlenbeck gauge with constant $\tilde{C}$ by some gauge transformation $u_{i} \in \mathcal{G}^{2, p}(B)$. Then there exists $u \in \mathcal{G}^{2, p}(B)$ such that $u^{*} A$ is also in Uhlenbeck gauge with constant $\tilde{C}$.

Proof. We will show that there exist the limits $u_{i} \rightarrow u$ and $u_{i}^{*} A_{i}=: \tilde{A}_{i} \rightarrow \tilde{A}$ such that $u^{*} A=\tilde{A}$ and this is in Uhlenbeck gauge. 1.29

We can get a uniform bound on $\left\|\tilde{A}_{i}\right\|_{1, p}$ by first bounding the curvature, from lemma

$$
\left\|F_{A_{i}}\right\|_{p} \leq c\left(\left\|A_{i}\right\|_{1, p}+\left\|A_{i}\right\|_{p}^{2}\right) \leq c^{\prime}
$$

with uniform $c^{\prime}$ due to the $W^{1, p}$-convergence of the $A_{i}$, and then applying the Uhlenbeck gauge condition

$$
\left\|\tilde{A}_{i}\right\|_{1, p} \leq \tilde{C}\left\|F_{A_{i}}\right\|_{p} \leq c^{\prime} \tilde{C}
$$

\footnotetext{
${ }^{13}$ That is, we elevate both sides to the $p$-th power, integrate over $B$ with the Euclidean volume form and take the $\frac{1}{p}$ power, and then separate the terms, all of which can be done because the integral, $x \mapsto x^{p}$ and $x \mapsto x^{\frac{1}{p}}$ are subadditive.
} 
With this uniform bound, we may use the Banach-Alaoglu theorem 1.18 to find a subsequence weakly converging to some $\tilde{A} \in \mathcal{A}^{1, p}(B)$, and then from the compact embedding $W^{1, p} \hookrightarrow L^{2 p}$, a further subsequence ${ }^{14}$ also converges in the $L^{2 p}$-norm to $\tilde{A}$.

Then, since both $\left\|A_{i}\right\|_{1, p}$ and $\left\|\tilde{A}_{i}\right\|_{1, p}$ are uniformly bounded, lemma 1.33 gives us a subsequence $u_{i}$ such that it converges in the $C^{0}$-topology to some $u \in \mathcal{G}^{2, p}(B)$, and $u_{i}^{-1} \mathrm{~d} u_{i} \longrightarrow u^{-1} \mathrm{~d} u$ uniformly in the $L^{2 p}$-norm.

Thus we have

$$
u^{-1} \mathrm{~d} u \longleftarrow u_{i}^{-1} \mathrm{~d} u_{i}=\tilde{A}_{i}-u_{i}^{-1} A_{i} u_{i} \longrightarrow \tilde{A}-u^{-1} A u,
$$

where the second limit follows because the two terms converge, and the second term converges because of the continuity of the adjoint action. ${ }^{15}$ Equality follows from the uniqueness of the $L^{2 p}$-limit, and so $\tilde{A}=u^{*} A$.

Now, we check that $\tilde{A}$ is in Uhlenbeck gauge, i.e. check (2.1) and $(2.2)$ :

(i) All $\tilde{A}_{i}$ are already in Uhlenbeck gauge and thus satisfy $\mathrm{d}^{*} \tilde{A}_{i}=0$, and for all $\phi \in$ $C^{\infty}(B)$ such that $\left.\phi\right|_{\partial B}=0$

$$
\begin{aligned}
\left\langle\phi, \mathrm{d}^{*} \tilde{A}\right\rangle & =\int_{B} \phi \mathrm{d}^{*} \tilde{A} * 1=\int_{B} \phi *(\mathrm{~d}^{*} \tilde{A}-\underbrace{\mathrm{d}^{*} \tilde{A}_{i}}_{=0}) \\
& =\underbrace{*^{2}}_{= \pm 1} \int_{B} \phi \mathrm{d}\left(* \tilde{A}-* \tilde{A}_{i}\right) \stackrel{(*)}{=} \pm \int_{B} \mathrm{~d} \phi \wedge *(\underbrace{\tilde{A}-\tilde{A}_{i}}_{\rightarrow 0}) \longrightarrow 0,
\end{aligned}
$$

where $(*)$ follows from the following computation (where we write $\alpha \in \Omega^{1}(B)$ ), using Stokes's theorem 1.20 and $\left.\phi\right|_{\partial B}=0$

$$
0=\int_{\partial B} \phi * \alpha=\int_{B} \mathrm{~d}(\phi * \alpha)=\int_{B} \mathrm{~d} \phi \wedge * \alpha+(-1)^{n-1} \int_{B} \phi \wedge \mathrm{d} * \alpha
$$

Since $C_{\delta}^{\infty}(B)$ is dense in $L^{p}(B)$, this proves that $\mathrm{d}^{*} \tilde{A}=0$.

(ii) Similarly, $\left.* \tilde{A}_{i}\right|_{\partial B}=0$ and this is also preserved under the weak $W^{1, p}$-limit, as we show. For any $\phi \in C^{\infty}(\partial B)$ we may extend it to some $\Phi \in C^{\infty}(B)$, and so we have

$$
\begin{aligned}
\left\langle\phi,\left.* \tilde{A}\right|_{\partial B}\right\rangle & =\left.\int_{\partial B} \phi * \tilde{A}\right|_{\partial B}=\left.\int_{\partial B} \Phi * \tilde{A}\right|_{\partial B}=\left.\int_{\partial B} \Phi *\left(\tilde{A}-\tilde{A}_{i}\right)\right|_{\partial B} \\
& \stackrel{(*)}{=} \int_{B} \mathrm{~d}\left(\Phi *\left(\tilde{A}-\tilde{A}_{i}\right)\right)=\int_{B} \mathrm{~d} \Phi *\left(\tilde{A}-\tilde{A}_{i}\right) \pm \int_{B} \Phi \mathrm{d} *\left(\tilde{A}-\tilde{A}_{i}\right) \\
& =\int_{B} \mathrm{~d} \Phi \wedge *\left(\tilde{A}-\tilde{A}_{i}\right) \pm \int_{B} \Phi *\left(* \mathrm{~d} *\left(\tilde{A}-\tilde{A}_{i}\right)\right) \\
& =\int_{B} \mathrm{~d} \Phi \wedge * \underbrace{\left(\tilde{A}-\tilde{A}_{i}\right)}_{\rightarrow 0} \pm \int_{B} \Phi \underbrace{\left(\mathrm{d}^{*} \tilde{A}-\mathrm{d}^{*} \tilde{A}_{i}\right)}_{=0}
\end{aligned}
$$

where in $(*)$ again we used Stokes's theorem 1.20 . This then shows that $\left.* \tilde{A}\right|_{\partial B}=0$ as we wished.

\footnotetext{
${ }^{14}$ We keep the same labelling $i \in \mathbb{N}$ for the subsequence.

${ }^{15}$ See calculation in proof of lemma 1.31 .
} 
(iii) Let $s=p$ or $q$. We may write

$$
\|\tilde{A}\|_{1, s} \leq \liminf _{i \rightarrow \infty}\left\|\tilde{A}_{i}\right\|_{1, s} \leq \tilde{C} \liminf _{i \rightarrow \infty}\left\|F_{A_{i}}\right\|_{s} \leq \tilde{C}\left\|F_{A}\right\|_{s}
$$

and this gives us our result. The first inequality is true because any norm is sequentially weakly lower semicontinuous; the second follows from $\tilde{A}_{i}$ being in Uhlenbeck gauge; and the third follows from the continuity of the $L^{s}$-energy functional on $\mathcal{A}^{1, s}$ from lemma 1.29 , and the convergence $A_{i} \stackrel{1, p}{\longrightarrow} A$, which also implies there is convergence in $W^{1, q}$ because for $p \geq q$ and $B$ of finite volume, $W^{1, q}(B) \hookrightarrow W^{1, p}(B)$.

\section{(3) $S_{\varepsilon}$ is open}

We would like to show that for any $A \in \mathcal{S}_{\varepsilon}$ there is a neighbourhood of $A$ in $\mathcal{A}_{\varepsilon}$ contained in $\mathcal{S}_{\varepsilon}$. Instead, it is simpler to find a neighbourhood of $A_{0}:=u^{*} A$ in $\mathcal{A}^{1, p}(B)$ made up of connections which can be put in Uhlenbeck gauge, pull it back by $u^{-1}$ to a neighbourhood of $A$ in $\mathcal{A}^{1, p}(B)$ and intercept it with $\mathcal{A}_{\varepsilon}$ to get what we need. This string of operations makes sense because $\mathcal{G}^{2, p}(B)$ acts continuously on $\mathcal{A}^{1, p}(B)$ (see lemma 1.31) and is closed under compositions. Since the energy $\mathcal{E}(A)$ is gauge invariant, we can forget about the original connection $A$ and work with an arbitrary connection in Uhlenbeck gauge.

We will make use of the implicit function theorem for a suitable operator between Banach spaces, which will yield a neighbourhood of connections around $A_{0}$ which satisfy (2.1) in the Uhlenbeck gauge definition. We can then ask for a bit more from the solutions so that the connections satisfy condition (2.2), owing to a a priori estimates.

Before moving on to the proof, it will be important to establish some inequalities with uniform constants. Since the $W^{1, p}$-norm only depends on the metric, its inverse and first derivatives, control over the $W^{1, \infty}$-norm of the metric is enough to guarantee that if $\|g-\mathbb{1}\|_{1, \infty} \leq \delta$ for small enough $\delta$, we get

$$
\frac{1}{2}\|\alpha\|_{g ; 1, p} \leq\|\alpha\|_{\mathbb{I} ; 1, p} \leq 2\|\alpha\|_{g ; 1, p}, \quad \forall \alpha \in W^{1, p}\left(B, T^{*} B\right) .
$$

If we choose $\delta$ such that this equivalence is valid for the $W^{1, p}, W^{1, q}$ and $L^{r}$-norms (with $r=r(n, p, q)$ from lemma A.9) at the same time, we moreover get uniform constants in the estimates between these spaces, by first using the appropriate estimate with the Euclidean metric $\mathbb{1}$, and then using $(2.3)$. This means that throughout the next proofs, the constants $C_{r n p q}$ coming from lemma A.9 and $C_{W}$ coming from the Sobolev embeddings will not depend on the metric.

Lemma 2.7 (A priori estimates). There exist positive constants $\delta, \tilde{C}$, and $\Lambda$ such that for every metric $g$ satisfying $\|g-\mathbb{1}\|_{1, \infty} \leq \delta$ the following holds:

Let $A \in \mathcal{A}^{1, p}(B)$ be such that $\mathrm{d}^{*} A=0$ on $B$ and $\left.* A\right|_{\partial B}=0$, and $\|A\|_{r} \leq \Lambda$, where $r=r(n, p, q)$ from lemma A.g. Then

$$
\begin{gathered}
\|A\|_{1, p} \leq \tilde{C}\left\|F_{A}\right\|_{p}, \\
\|A\|_{1, q} \leq \tilde{C}\left\|F_{A}\right\|_{q},
\end{gathered}
$$

that is, $A$ is in Uhlenbeck gauge with constant $\tilde{C}$. 
Proof. As we are working over the ball in Euclidean space and $\left.* A\right|_{\partial B}=0$, it follows from theorem 1.26 that for all $1<s<\infty$,

$$
\|A\|_{1, s} \leq C_{g}(\|\mathrm{~d} A\|_{s}+\underbrace{\left\|\mathrm{d}^{*} A\right\|_{s}}_{=0}),
$$

where $C_{g}$ depends on the metric $g$ on $B$; however, we also know that the constant depends $W^{1, \infty}$-continuously on the metric, and so we may choose $\delta$ such that there is a uniform constant $C$ for all metrics $\|g-\mathbb{1}\|_{1, \infty} \leq \delta$, and thus

$$
\|A\|_{1, s} \leq C\|\mathrm{~d} A\|_{s} .
$$

Now, looking at the curvature as $\mathrm{d} A=F_{A}-\frac{1}{2}[A \wedge A]$,

$$
\|A\|_{1, s} \leq C\left(\left\|F_{A}\right\|_{s}+\frac{1}{2}\|[A \wedge A]\|_{s}\right)
$$

and we would like to get rid of this $\|[A \wedge A \|$ term somehow. There is a clever trick to make it so that we can absorb this term into the constant and the left hand side. We take $s=p$ or $q$ and use lemma A.9 in the following:

$$
\begin{aligned}
\frac{1}{2}\|[A \wedge A]\|_{s} & \leq\|[A \wedge A]\|_{\mathbb{1} ; s} \\
& \stackrel{(1)}{\leq}\|2|A| \cdot|A|\|_{\mathbb{1} ; s} \\
& \leq 2 C_{r n p q}\|A\|_{\mathbb{1} ; r}\left\||A|_{\mathbb{1}}\right\|_{\mathbb{1} ; 1, s} \\
& \stackrel{(2)}{\leq} 8 C_{r n p q}\|A\|_{r}\|A\|_{1, s} \\
& \leq 8 C_{r n p q} \Lambda\|A\|_{1, s},
\end{aligned}
$$

where in (1) we used that $|[A \wedge A]|_{\mathbb{1}} \leq 2|A|_{\mathbb{1}}^{2}$ (see e.g. (1.28) and proof of lemma 1.29), and in (2) we used that $|\nabla| A|| \leq|\nabla A|$ (see lemma A.10). Since we used lemma A.9 for the Euclidean metric and then used the equivalence of the norms $(2.3), C_{r n p q}$ is metric independent and we may absorb it into $C$,

$$
\|A\|_{1, s} \leq C\left(\left\|F_{A}\right\|_{s}+\Lambda\|A\|_{1, s}\right),
$$

and now all that is left to do is take $\Lambda=\frac{1}{2 C}$ and combine the $\|A\|_{1, s}$ terms, and the result follows for $\tilde{C}=2 C$.

We will need to keep track of this extra condition $\|A\|_{r} \leq \Lambda$ while solving the boundary value problem posed by (2.1), and this will appear as a bound $\lambda$ on $X$ for a gauge transformation $\exp (X)$, so that a transformed connection $\exp (X)^{*} A$ will satisfy this $\Lambda$ bound on its $L^{r}$-norm.

Lemma 2.8. There exists $\delta>0$ such that for every constant $\tilde{C}>0$ there exists $\varepsilon>0$ such that for every metric $g$ satisfying $\|g-\mathbb{1}\|_{2, \infty} \leq \delta$ the following is true:

Let $A_{0} \in \mathcal{A}^{1, p}(B)$ be in Uhlenbeck gauge with constant $\tilde{C}$ and energy $\mathcal{E}(A) \leq \varepsilon$; then for all $\lambda>0$ there exists $R>0$ such that for every connection $A \in \mathcal{A}^{1, p}(B)$ with $\left\|A-A_{0}\right\|_{1, p} \leq$ $R$ there is a solution $X \in W^{2, p}(B, \mathfrak{g})$ of

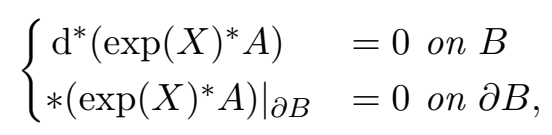

with $\|X\|_{2, p} \leq \lambda$. 
Proof. As explained, we will use the implicit function theorem A.3. Let us define our Banach spaces:

First, define the space

$$
W_{m}^{2, p}(B, \mathfrak{g}):=\left\{X \in W^{2, p}(B, \mathfrak{g}): \int_{B} X=0\right\},
$$

which is a closed subspace ${ }^{16}$ of a Banach space, and therefore also Banach. Next, define

$$
W_{\partial}^{1, p}(B, \mathfrak{g}):=\frac{W^{1, p}(B, \mathfrak{g})}{W_{\delta}^{1, p}(B, \mathfrak{g})}
$$

where $W_{\delta}^{1, p}(B, \mathfrak{g})$ is the closure in the $W^{1, p}$-norm of the subspace of sections which vanish at the boundary of $B$. This is a boundary value space, whose elements are equivalence classes of functions on $B$ which coincide on the boundary $\partial B$. With the quotient norm

$$
\|\phi\|_{W_{\partial}^{1, p}}(B, \mathfrak{g})=\inf \left\{\|\Phi\|_{1, p}: \Phi \in W^{1, p}(B, \mathfrak{g}) \text { and }\left.\Phi\right|_{\partial B}=\phi\right\},
$$

$W_{\partial}^{1, p}(B, \mathfrak{g})$ is a Banach space. Now define

$$
\mathcal{Z}=\left\{(f, \phi) \in L^{p}(B, \mathfrak{g}) \times W_{\partial}^{1, p}(B, \mathfrak{g}): \int_{B} f+\int_{\partial B} \phi=0\right\} .
$$

Note that $L^{p}(B, \mathfrak{g}) \times W_{\partial}^{1, p}(B, \mathfrak{g})$ with the direct sum norm is a Banach space, and $\mathcal{Z}$ is a closed subspace, and therefore Banach. To see that

$$
\int_{B} f+\int_{\partial B} \phi=0
$$

is a closed condition, note that using the trace theorem 1.19 we get

$$
\int_{\partial B}|\phi|=\|\phi\|_{1(\partial B)} \leq C\|\Phi\|_{1, p(B)} \leq C\left(\|\phi\|_{W_{\partial}^{1, p}}+\varepsilon\right)
$$

for some choice of $\Phi$ such that $\left.\Phi\right|_{\partial B}=\phi$, which is enough to see that $(2.4)$ is preserved under limits. Finally, $\mathcal{A}^{1, p}(B)$ is a Sobolev space and therefore clearly Banach.

We will use the implicit function theorem on the operator

$$
\begin{aligned}
F: \mathcal{A}^{1, p}(B) \times W_{m}^{2, p}(B, \mathfrak{g}) \longrightarrow \mathcal{Z} & \longrightarrow \\
(A, X) & \mapsto\left(\mathrm{d}^{*}\left(\exp (X)^{*} A\right),\left.*\left(\exp (X)^{*} A\right)\right|_{\partial B}\right) .
\end{aligned}
$$

That $F$ is a continuous map into $L^{p}(B, \mathfrak{g}) \times W_{\partial}^{1, p}(B, \mathfrak{g})$ follows from the facts that $\exp X \in$ $\mathcal{G}^{2, p}(B)$ for $X \in W^{2, p}(B, \mathfrak{g}$ ) by definition (see lemma 1.21), the map $X \mapsto \exp X$ is continuous, and the gauge action $\mathcal{G}^{2, p} \times \mathcal{A}^{1, p} \rightarrow \mathcal{A}^{1, p}$ is continuous (see lemma 1.31). That it maps into $\mathcal{Z}$ can be checked with Stokes's theorem (lemma 1.20 : for $\alpha=(\exp X)^{*} A{ }^{17}$

$$
\int_{B} \mathrm{~d}^{*} \alpha * 1=-\int_{B} \mathrm{~d} * \alpha=-\left.\int_{\partial B} * \alpha\right|_{\partial B}
$$

\footnotetext{
${ }^{16} \int_{B} X=0$ is a (clearly linear and) closed condition, which is easily seen taking limits in each component of $\mathfrak{g}$.

${ }^{17}$ Use $k=n$ in the formulas $*^{2}=(-1)^{k(n-k)}$ and $\mathrm{d}^{*}=-(-1)^{n(k-1)}$.
} 
so that 2.4 is indeed satisfied by $(f, \phi)$ in the image of $F$. Thus, $F$ is a continuous operator between Banach spaces.

Now, knowing that $F\left(A_{0}, 0\right)=0$, what we want is an open set around $A_{0}$ in $\mathcal{A}^{1, p}(B)$ and some continuous map $f$ on this open set to a neighbourhood of $0 \in W_{m}^{2, p}(B, \mathfrak{g})$ such that $F(A, f(A))=0$, and this map will permit us to control the norm of $X$. Under these circumstances, we need to analyse the derivative of $F$ with respect to the second variable $X$. The partial derivative $\left.\partial_{X} F\right|_{(A, X)}$ is the Fréchet derivative of the map $F(A, \cdot)$, which is a linear map

$$
\left.\partial_{X} F\right|_{(A, X)}: W_{m}^{2, p}(B, \mathfrak{g}) \rightarrow \mathcal{Z} .
$$

We check that this map exists, is continuous and is bijective at $\left(A_{0}, 0\right)$. First, note that since the chain rule is valid for the Fréchet derivative, and $\mathrm{d}, *$ and $\left.\right|_{\partial B}$ are linear maps, we need only look at the Fréchet derivative of the gauge action $X \mapsto(\exp (X))^{*} A$, which we will call $\mathcal{G}(A, X): W_{m}^{2, p}(B, \mathfrak{g}) \rightarrow \mathcal{A}^{1, p}(B)$, and then

$$
\left.\partial_{X} F\right|_{(A, X)}: \xi \mapsto\left(\mathrm{d}^{*}(\mathcal{G}(A, X) \xi),\left.*(\mathcal{G}(A, X) \xi)\right|_{\partial B}\right) .
$$

We will show that the linearization of the gauge action is

$$
\mathcal{G}(A, X) \xi=\mathrm{d} \xi+\mathrm{d}_{\exp (-X)} \operatorname{Ad}\left(\mathrm{d}_{-X} \exp (-\xi)\right) A,
$$

which is to say that

$$
\lim _{\|\xi\| \rightarrow 0} \frac{\left|\exp (X+\xi)^{*} A-\exp (X)^{*} A-\mathrm{d} \xi-\mathrm{d}_{\exp (-X)} \operatorname{Ad}\left(\mathrm{d}_{-X} \exp (-\xi)\right) A\right|}{\|\xi\|}=0 .
$$

We can rewrite this in a more suggestive format,

$$
\begin{gathered}
\exp (X+\xi)^{*} A-\exp (X)^{*} A-\mathrm{d} \xi-\mathrm{d}_{\exp (-X)} \operatorname{Ad}\left(\mathrm{d}_{-X} \exp (-\xi)\right) A \\
=\operatorname{Ad}_{\exp (X+\xi)^{-1} A+\exp (X+\xi)^{-1} \mathrm{~d} \exp (X+\xi)}-\operatorname{Ad}_{\exp X^{-1}} A-\exp X^{-1} \mathrm{~d} \exp X \\
-\mathrm{d} \xi-\mathrm{d}_{\exp (-X)} \operatorname{Ad}\left(\mathrm{d}_{-X} \exp (-\xi)\right) A \\
=\operatorname{Ad}_{\exp (X+\xi)^{-1}} A-\operatorname{Ad}_{\exp X^{-1}} A-\mathrm{d}_{\exp (-X)} \operatorname{Ad}\left(\mathrm{d}_{-X} \exp (-\xi)\right) A,
\end{gathered}
$$

and observe that this expression is exactly the expression for the derivative of $\operatorname{Ad}_{\exp Y}$,

$$
\begin{aligned}
\mathrm{d}_{(-X)}\left(\operatorname{Ad}_{\exp }\right)(-\xi) & =\mathrm{d}_{(-X)}(\operatorname{Ad} \circ \exp )(-\xi) \\
& =\mathrm{d}_{\exp (-X)} \operatorname{Ad}\left(\mathrm{d}_{(-X)} \exp (-\xi)\right) .
\end{aligned}
$$

Thus the limit is indeed zero. Moreover, $\mathcal{G}(A, X)$ is clearly continuous.

To check bijectivity of $\left.\partial_{X} F\right|_{\left(A_{0}, 0\right)}$ we simplify the expression

$$
\begin{aligned}
\mathcal{G}\left(A_{0}, 0\right) \xi & =\mathrm{d} \xi+\mathrm{d}_{\mathbb{1}} \operatorname{Ad}\left(\mathrm{d}_{0} \exp (-\xi)\right) A_{0} \\
& \stackrel{(*)}{=} \mathrm{d} \xi+\mathrm{d}_{\mathbb{1}} \operatorname{Ad}(-\xi) A_{0} \\
& =\mathrm{d} \xi+\operatorname{ad}(-\xi) A_{0} \\
& =\mathrm{d} \xi-\left[\xi \wedge A_{0}\right]
\end{aligned}
$$

where $(*)$ follows because the differential of the exponential map at zero is the identity. Now, we must look at $\mathrm{d}^{*} \mathcal{G}\left(A_{0}, 0\right) \xi$ and $\left.* \mathcal{G}\left(A_{0}, 0\right) \xi\right|_{\partial B}$. Note that because $\xi \in W_{m}^{2, p}(B, \mathfrak{g})$ is 
just a function, $*\left[\xi \wedge A_{0}\right]=\left[\xi \wedge * A_{0}\right]$, and remember that $A_{0}$ is in Uhlenbeck gauge already. Then we calculate

$$
\begin{aligned}
-\mathrm{d}^{*}\left[\xi \wedge A_{0}\right] & =-(-* \mathrm{~d} *)\left[\xi \wedge A_{0}\right]=* \mathrm{~d}\left[\xi \wedge * A_{0}\right] \\
& =*\left[\mathrm{~d} \xi \wedge * A_{0}\right]+*\left[\xi \wedge \mathrm{d} * A_{0}\right] \\
& =*\left[\mathrm{~d} \xi \wedge * A_{0}\right]+[\xi \wedge \underbrace{* \mathrm{~d} * A_{0}}_{-\mathrm{d}^{*} A_{0}=0}] \\
& =*\left[\mathrm{~d} \xi \wedge * A_{0}\right] .
\end{aligned}
$$

Moreover, $\left.* A_{0}\right|_{\partial B}=0$ and so

$$
\left.*\left(\mathrm{~d} \xi-\left[\xi \wedge A_{0}\right]\right)\right|_{\partial B}=\left.* \mathrm{~d} \xi\right|_{\partial B} .
$$

Therefore

$$
\left.\partial_{X} F\right|_{A_{0}, 0} \xi=\left(\mathrm{d}^{*} \mathrm{~d} \xi+*\left[\mathrm{~d} \xi \wedge * A_{0}\right],\left.* \mathrm{~d} \xi\right|_{\partial B}\right) .
$$

We write $\left.\partial_{X} F\right|_{A_{0}, 0}=T+S$, where

$$
T=\left(\Delta \xi,\left.* \mathrm{~d} \xi\right|_{\partial B}\right) \text { and } S=\left(*\left[\mathrm{~d} \xi \wedge * A_{0}\right], 0\right) .
$$

Note that $T$ is the operator for the inhomogeneous Neumann problem ${ }^{18}$ From theorem A.6. $\mathcal{Z}$ is exactly the space of functions $(f, g)$ for which the Neumann problem has solution, and so $T$ is surjective onto $\mathcal{Z}$; moreover, the solutions are unique up to additive constant, and so the additional condition on the domain $W_{m}^{2, p}(B, \mathfrak{g})$ that $\int_{B} \xi=0$ makes $T$ injective. Furthermore, by theorem A.7. the inverse of this operator is bounded for every metric $W^{2, \infty}$-close to the identity. Choosing an appropriate $\delta$, we can make $\left\|T^{-1}\right\| \leq C_{T}$ for some constant $C_{T}$ which is independent of the metric. Now, if we find a bound for $S$, lemma A.5 gives the bijectivity of $T+S$. Calculate

$$
\begin{aligned}
\left|*\left[\mathrm{~d} \xi \wedge * A_{0}\right]\right| & =\left|* \sum\left[\partial_{i} \xi, A_{j}\right] \mathrm{d} x_{i} \wedge * \mathrm{~d} x_{j}\right|=\left|* \sum\left[\partial_{i} \xi, A_{j}\right] g^{i j} * 1\right| \\
& =\left|\sum g^{i j}\left[\partial_{i} \xi, A_{j}\right]\right| \leq \sum\left|g^{i j}\left[\partial_{i} \xi, A_{j}\right]\right| \\
& \leq \sum\left|g^{i j}\right|\left|\partial_{i} \xi\right|\left|A_{j}\right| \leq \underbrace{\max \left|g^{i j}\right|}_{=:\left|g^{-1}\right|} \sum\left|\partial_{i} \xi\right|\left|A_{j}\right| \\
& =\left|g^{-1}\right||\mathrm{d} \xi|_{\mathbb{1}}\left|A_{0}\right|_{\mathbb{1}} .
\end{aligned}
$$

Then, because $\left|g^{-1}\right| \leq\left\|g^{-1}\right\|_{\infty}$, we can use lemma A.4 and require $\delta \leq \frac{1}{2}$ such that $\left|g^{-1}\right| \leq$ $\left(1-\|g-\mathbb{1}\|_{\infty}\right)^{-1} \leq 2$, and now we apply this in inequality $(*)$ below:

$$
\begin{aligned}
\|S \xi\|_{\mathcal{Z}} & =\left\|*\left[\mathrm{~d} \xi \wedge * A_{0}\right]\right\|_{g ; p} \\
& \leq 2\left\|*\left[\mathrm{~d} \xi \wedge * A_{0}\right]\right\|_{\mathbb{1} ; p} \\
& =2\left(\int_{B}\left|*_{g}\left[\mathrm{~d} \xi \wedge *_{g} A_{0}\right]\right|_{\mathbb{1}}^{p}\right)^{\frac{1}{p}} \\
& \stackrel{(*)}{\leq} 4\left(\int_{B}\left(|\mathrm{~d} \xi|_{\mathbb{1}}\left|A_{0}\right|_{\mathbb{1}}\right)^{p}\right)^{\frac{1}{p}}
\end{aligned}
$$

${ }^{18}$ See appendix $\mathrm{A}$ the section on the Neumann problem. 


$$
\begin{aligned}
& =4\left\||\mathrm{~d} \xi|_{\mathbb{I}}\left|A_{0}\right|_{\mathbb{1}}\right\|_{\mathbb{1} ; p} \\
& \leq 4 C_{r n p q}\left\||\mathrm{~d} \xi|_{\mathbb{1}}\right\|_{\mathbb{I} ; 1, p}\left\|\left|A_{0}\right|_{\mathbb{1}}\right\|_{\mathbb{1} ; 1, q} \\
& \stackrel{(1)}{\leq} 4 C_{\text {rnpq }}\|\xi\|_{\mathbb{1} ; 2, p}\left\|A_{0}\right\|_{\mathbb{1} ; 1, q} \\
& \leq 16 C_{r n p q}\|\xi\|_{2, p}\left\|A_{0}\right\|_{1, q},
\end{aligned}
$$

where the factors of 2 come from the equivalence of the norms 2.3 on $B$, the constants $C_{r n p q}$ come from lemma A.9 as usual, and in (1) we use lemma A.10 for $|\nabla| A|| \leq|\nabla A|$ and that on sections $\xi: B \rightarrow \mathfrak{g}, \nabla=\mathrm{d}$ so $\nabla \mathrm{d} \xi=\nabla^{2} \xi$,

Now, let $\tilde{C}$ be given and choose $\varepsilon=\left(32 C_{r n p q} \tilde{C} C_{T}\right)^{-q}$. Since $A_{0}$ is in Uhlenbeck gauge with energy $\mathcal{E}_{q}\left(A_{0}\right) \leq \varepsilon$ and constant $\tilde{C}$, we can use $\left\|A_{0}\right\|_{1, q} \leq \tilde{C}\left\|F_{A_{0}}\right\|_{q} \leq \varepsilon^{\frac{1}{q}} \tilde{C}$ and at last

$$
\|S \xi\|_{\mathcal{Z}} \leq 16 C_{r n p q} \tilde{C} \varepsilon^{\frac{1}{q}}\|\xi\|_{2, p}=\frac{1}{2 C_{T}}\|\xi\|_{2, p} .
$$

This means that $S$ and $T$ satisfy the hypotheses from lemma A.5 namely that $T$ is bijective with bounded inverse and $\|S\|\left\|T^{-1}\right\|=\frac{1}{2}<1$, and therefore $\left.\partial_{X} F\right|_{A_{0}, 0}=T+S$ is bijective.

Finally, this means that $F$ satisfies all conditions to the implicit function theorem A.3 around $\left(A_{0}, 0\right)$, and therefore there are neighbourhoods $U$ around $A_{0}$ and $V$ around 0 , and a continuous map $f: U \rightarrow V$ such that $F(A, f(A))=0$, which implies that $\exp (f(A))^{*} A$ is in Uhlenbeck gauge for all $A \in U \subseteq \mathcal{A}^{1, p}(B, \mathfrak{g})$. Furthermore, to get the bound $\|X\|_{2, p} \leq \lambda$, it suffices to take a ball with sufficiently small radius $R$ within $f^{-1}\left(B_{\lambda}(0) \cap V\right) \subseteq U$, so that $f: B_{R}\left(A_{0}\right) \rightarrow B_{\lambda}(0)$.

Lemma 2.7 fixes the constant $\tilde{C}$ we will use for the Uhlenbeck gauge, and this in turn fixes $\varepsilon$, which is defined in terms of $\tilde{C}$ in the proof of lemma 2.8. Then for a connection $A_{0}$ in Uhlenbeck gauge with constant $\tilde{C}$ and energy $\mathcal{E}_{q} \leq \varepsilon$, we find $R$ such that for every connection $A \in B_{R}\left(A_{0}\right) \subseteq \mathcal{A}^{1, p}(B)$, there is a gauge transformation $\exp (X)$ such that $(\exp X)^{*} A$ satisfies $(2.1)$, the first condition of the Uhlenbeck gauge, and we get a bound $\|X\|_{2, p} \leq \lambda$.

All that is left to do now is check that these solutions $(A, X)$ satisfy $\left\|\exp (X)^{*} A\right\|_{r} \leq \Lambda$, so that they can be shown to satisfy $(2.2)$ by lemma 2.7 and finally all of $B_{R}\left(A_{0}\right)$ can be put into Uhlenbeck gauge with constant $C$. Therefore, we show this now: first, we have

$$
\left\|\exp (X)^{*} A\right\|_{r} \leq\left\|\operatorname{Ad}_{\exp (X)} A\right\|_{r}+\|\exp (-X) \mathrm{d} \exp (X)\|_{r} .
$$

Because the inner product on $\mathfrak{g}$ is Ad-invariant, the first term is just $\|A\|_{r}$ and we have

$$
\begin{aligned}
\|A\|_{r} & \leq\left\|A-A_{0}\right\|_{r}+\left\|A_{0}\right\|_{r} \\
& \stackrel{(*)}{\leq} 4 C_{W}\left(\left\|A-A_{0}\right\|_{1, p}+\left\|A_{0}\right\|_{1, q}\right) \\
& \leq 4 C_{W}(R+\tilde{C} \varepsilon),
\end{aligned}
$$

where $(*)$ follows from the equivalence of metrics $(2.3)$ and the Sobolev embeddings $L^{r} \hookrightarrow$ $W^{1, p}$ and $L^{r} \hookrightarrow W^{1, q}$. As for the second term, we can write

$$
\exp (-X(x)) \mathrm{d} \exp (X(x))=\exp (-X(x)) \mathrm{d}_{X} \exp \circ \mathrm{d} X
$$

and note that, since $2 p>n$, the embedding $W^{2, p} \hookrightarrow C^{0}$ provides control over the $C^{0}$-norm of $X, \sup _{x \in B}|X(x)| \leq \lambda$. Therefore, for sufficiently small $\lambda$, for every point $x \in B$ the map 
$\exp (-X(x)) \mathrm{d}_{X(x)} \exp : \mathfrak{g} \rightarrow \mathfrak{g}$ is arbitrarily close to $\exp (0) \mathrm{d}_{0} \exp =\mathrm{d}_{0} \exp =\mathbb{1}_{\mathfrak{g}}$, which means that its norm can be bounded by 2 . Thus,

$$
\begin{aligned}
\|\exp (-X) \mathrm{d} \exp (X)\|_{r} & =\left\|\exp (-X) \mathrm{d}_{X} \exp \circ \mathrm{d} X\right\|_{r} \\
& \leq 2\|\mathrm{~d} X\|_{r} \leq 4\|\mathrm{~d} X\|_{\mathbb{1} ; r} \\
& \leq 4 C_{W}\|\mathrm{~d} X\|_{\mathbb{1} ; 1, p} \\
& \leq 8 C_{W}\|\mathrm{~d} X\|_{1, p} \\
& \leq 8 C_{W}\|X\|_{2, p} \leq 8 C_{W} \lambda .
\end{aligned}
$$

For the first term, $4 C_{W}$ does not depend on the metric, and $\tilde{C}$ has already been fixed; for the second term, $8 C_{W}$ does not depend on the metric, and so putting both terms together we have

$$
\left\|\exp (X)^{*} A\right\|_{r} \leq c(R+\varepsilon+\lambda)
$$

for some uniform constant $c$. Given $\tilde{C}$, we chose $\varepsilon$ as a uniform constant which can be made smaller. The constant $\lambda$ is arbitrary, and so once again can be made even smaller. Finally, while $R$ depends on $\lambda$ and the metric on $B$, we can put a uniform upper bound on it which can also be made smaller, and furthermore making $\lambda$ smaller only makes $R$ smaller as well. Then, making each term small as needed, we may bound $\left\|\exp (X)^{*} A\right\|_{r}$ by $\Lambda$ for all metrics $\|g-\mathbb{1}\|_{1, \infty} \leq \delta$.

This ends the proof of the gauge fixing lemma on $B$. Let us recapitulate the choices of constants that were made during the proof of the theorem:

- For proving that $S_{\varepsilon}$ is connected and closed, there was no need to fix anything.

- Pick a $\delta$ small enough such that if $\|g-\mathbb{1}\|_{1, \infty} \leq \delta$, there is equivalence of norms 2.3 with different metrics on $B$ for $W^{1, p}, W^{1, q}$ and $L^{r}$ with $r=r(n, p, q)$ from lemma A.9.

- In the implicit function step, pick $\delta$ possibly smaller to guarantee bijectivity of the derivative of the operator $F$ defined. Then, for any $\tilde{C}$ given, independent of the metric, choose $\varepsilon$ appropriately, which will also be uniform. For each metric and every $\lambda$, there is $R(\lambda, g)$ such that $\|X\|_{2, p} \leq \lambda$ for $X=f(A)$ and $\left\|A-A_{0}\right\|_{1, p} \leq R$.

- The Uhlenbeck gauge constant $\tilde{C}$ is fixed with the a priori estimates. Along with it, $\delta$ is picked once again for an estimate from theorem 1.26 to hold with uniform constant, and we find a bound $\Lambda$ on the $L^{r}$-norm of the connection which guarantees that these a priori estimates hold.

- Finally, when checking that the solution to the implicit function problem has $L^{r}$-bound $\Lambda$, we find that it is bounded by $R(\lambda, g)+\varepsilon+\lambda$, where $\lambda$ can be chosen arbitrarily, $\varepsilon$ was already a uniform constant which can be made smaller, and we may choose a uniform bound $R$ for $R(\lambda, g)$ such that the $\lambda$ bound holds for each metric and a given $\lambda$. Then $R, \varepsilon$ and $\lambda$ can be made small enough for the $\Lambda$ bound to hold. 


\section{Chapter 3}

\section{WEAK AND STRONG COMPACTNESS}

The notion of sequential compactness is an extremely important one, and from a first course in real analysis one learns that "a bounded sequence in $\mathbb{R}$ has a convergent subsequence" and that in $\mathbb{R}^{n}$ it is equivalent for a set to be compact, sequentially compact or closed and bounded. As one moves to studying functional analysis, things start to get more complicated. The closed unit ball is no longer compact if it is in an infinite dimensional Banach space, weaker topologies become important and one gets the Banach-Alaoglu theorem (in its original form). The notion of equicontinuity, which one may first encounter when studying the Arzelà-Ascoli theorem on $\mathbb{R}^{n}$, generalizes to metric spaces; the Banach-Steinhaus theorem, one of the fundamental results in functional analysis, is also known as the uniform boundedness principal and states precisely that a pointwise bounded family of continuous linear operators between Banach spaces is equicontinuous.

Other than the gauge fixing lemma, which we proved in the previous chapter, the importance of Uhlenbeck's Connections with $L^{p}$ bounds on curvature [Uhl82] is a compactness theorem, which translates a uniform bound to convergence. A little more precisely, she proves the weak compactness theorem, which states that a sequence of connections with uniformly bounded curvatures has a weakly convergent subsequence. Along with the regularity of the Yang-Mills equation provided by the gauge fixing lemma, the strong compactness theorem emerges as a corollary, and it states that a sequence of Yang-Mills connections with uniformly bounded curvature has a subsequence which converges uniformly.

The goal of this chapter is to prove these two theorems. First, we begin by commenting on compactness for geodesics and flat connections. The case of the geodesics is simple and instructive. The case of the flat connections is the opposite: while these connections do have a uniform bound on curvature (namely, zero), the usual proof of the compactness result for flat connections does not need this, instead using an identification with a specific class of homomorphisms. We include a sketch of this proof here for completeness, but also to show that not all compactness theorems emerge in the same way. Finally, note that the weak compactness theorem can be seen as a generalization of the result for flat connections.

\section{Geodesics}

Let $(M, g)$ be a Riemannian manifold, and consider curves $\gamma:[0,1] \rightarrow M$ with fixed endpoints $\gamma(0), \gamma(1) \in M$. A geodesic will be one such curve that minimizes length, so it is 
a critical point of the length functional

$$
\mathcal{L}(\gamma)=\int_{0}^{1}|\nabla \gamma| \mathrm{d} t
$$

It is also a critical point of the $L^{2}$-energy

$$
\mathcal{E}(\gamma)=\int_{0}^{1}|\nabla \gamma|^{2} \mathrm{~d} t
$$

For $a, b \in[0,1]$, the distance between two points on the curve is

$$
\begin{aligned}
\mathrm{d}(\gamma(a), \gamma(b))=\int_{a}^{b}|\nabla \gamma| \mathrm{d} t & \left.\left.\stackrel{(*)}{\leq}\left|\int_{a}^{b} 1 \mathrm{~d} t\right|^{\frac{1}{2}}\left|\int_{a}^{b}\right| \nabla \gamma\right|^{2} \mathrm{~d} t\right|^{\frac{1}{2}} \\
& \leq|a-b|^{\frac{1}{2}} \mathcal{E}(\gamma)^{\frac{1}{2}}
\end{aligned}
$$

where $(*)$ is the Cauchy-Schwartz inequality. Using this now it is easy to see how uniform bounds on energy can lead to compactness properties. Let $\left\{\gamma_{i}\right\}_{i \in \mathbb{N}}$ be a sequence of paths $\gamma_{i}:[0,1] \rightarrow M$ with fixed endpoints and $\mathcal{E}\left(\gamma_{i}\right)<C$. Looking at $(M, g)$ as a metric space with this distance as the metric, we have that

$$
\mathrm{d}\left(\gamma_{i}(a), \gamma_{i}(b)\right) \leq C|a-b|^{\frac{1}{2}},
$$

which makes this sequence equicontinuous 1 . Therefore, by the Arzelà-Ascoli theorem, there is a uniformly convergent subsequence.

\section{Flat connections}

The previous example had a very clear procedure: uniform bounds lead to convergence. We now come back to gauge theory and look at flat connections on trivial vector bundles. It is a very classical result that the moduli space of flat $G$-connections is compact, but the usual way to prove it does not follow the same procedure. Instead, it hinges on the following lemma.

Lemma 3.1 (Cra15, corollary 1.29). Let $E \rightarrow M$ be a vector bundle with flat connections $\nabla$. Then for any $x, y \in M$, the induced parallel transport from $E_{x}$ to $E_{y}$ only depends on the homotopy class of the path from $x$ to $y$.

A connection is called $G$-connection if, for instance, the associated parallel transports induce isomorphisms of the fibres which are in $G$ as a subgroup of $G L\left(\mathbb{R}^{m}\right)$ (this will always be the case for $G$ compact), for $m$ the rank of the bundle. With this lemma, we can write the following identification.

Proposition 3.2 ([DK97], proposition 2.2.3). There is a one-to-one correspondence between conjugacy classes of homomorphisms $\rho: \pi_{1}(M) \rightarrow G$ and gauge equivalence classes of flat $G$-connections on $M$.

Sketch of proof. Let $\nabla$ be a flat $G$-connection, then the associated parallel transport induces a representation $\rho^{\nabla}: \pi_{1}(M, x) \rightarrow G$ by defining, for each loop $\gamma$ based at $x \in M$,

$$
\rho^{\nabla}(\lfloor\gamma\rceil)=P_{\gamma}^{\nabla} \in G \subseteq G L\left(E_{x}\right) .
$$

\footnotetext{
${ }^{1}$ For $\varepsilon>0$, take $\delta=(\varepsilon / C)^{2}$.
} 
If we prove compactness of the space of homomorphisms $\rho: \pi_{1}(M) \rightarrow G$ when $G$ is compact, then we are done. We will sketch this proof.

Lemma 3.3. Let $G$ be a compact Lie group, $M$ a smooth manifold. Then $\frac{\operatorname{Hom}\left(\pi_{1}(M), G\right)}{G}$ is compact.

Sketch of proof. We prove that $\operatorname{Hom}\left(\pi_{1}(M), G\right)$ is closed in $G^{\pi_{1}(M)}$ with the product topology, which is compact by Tychonoff's theorem and because $G$ is compact. Then $\operatorname{Hom}\left(\pi_{1}(M), G\right)$ is compact, as is quotient.

Let $f: \pi_{1}(M) \rightarrow G$ be a function which is not a homomorphism: there exist $r, s \in \pi_{1}(M)$ such that $f(r) f(s) \neq f(r s)$. Take $U_{f(r) f(s)}, U_{f(r s)}$ neighbourhoods of $f(r) f(s)$ and $f(r s)$ which do not intersect. Since $G$ is a topological group, the multiplication is continuous and the preimage of $U_{f(r) f(s)}$ gives neighbourhoods of $f(r)$ and $f(s), U_{f(r)}$ and $U_{f(s)}$, respectively. Now, letting $p_{r}: G^{\pi_{1}(M)} \rightarrow G$ be the continuous projection $p_{r}(f)=f(r)$, we construct a neighbourhood of $f$ on $G^{\pi_{1}(M)}$,

$$
\mathcal{U}=p_{r}^{-1}\left(U_{f(r)}\right) \cap p_{s}^{-1}\left(U_{f(s)}\right) \cap p_{f(r s)}^{-1}\left(U_{f(r s)}\right),
$$

such that for any $g \in \mathcal{U}, g$ cannot be a homomorphism: $g(r s) \in U_{f(r s)}$ and $g(r) g(s) \in$ $U_{f(r) f(s)}$, and therefore $g(r s) \neq g(r) g(s)$. Thus, the complement of the homomorphisms is open and consequently $\operatorname{Hom}\left(\pi_{1}(M), G\right)$ is closed.

Corollary 3.4. The moduli space of flat $G$-connections on $M$ is compact.

\subsection{Weak compactness}

Without further ado, we come to the result. Let $M$ be a compact $n$-manifold with (possibly empty) boundary, and let $P \rightarrow M$ be a $G$-principal bundle.

Theorem 3.5 (Weak compactness). Let $\frac{n}{2}<p<\infty$. A sequence of connections in $\mathcal{A}^{1, p}(P)$ with uniform $L^{p}$-bound on the sequence of curvatures has a subsequence which is gauge equivalent to a weakly convergent sequence, with gauge transformations in $\mathcal{G}^{2, p}(P)$.

For the proof of this theorem we are essentially looking for a sequence of global gauges such that the gauged connections are uniformly $W^{1, p}$-bounded and will therefore, by the Banach-Alaoglu theorem, converge weakly. For that we will need the gauge fixing theorem 2.2 , and a patching lemma 3.10 which we will prove later, but roughly goes as follows.

Lemma 3.6 (cf. lemma 3.10). Two sets of transition functions describe isomorphic bundles if they are $C^{0}$-close. Furthermore, there are bounds on the gauge transformations relating these transition functions; for a sequence of transition functions and transformations, the bounds are uniform.

The gauge fixing lemma will give us a bundle atlas of $P \rightarrow M$ such that the local connections are in Uhlenbeck gauge, and therefore the uniform bound on the curvature will translate into a uniform bound on the connections. The gauge transformations resulting from the lemma do not necessarily patch up to a global gauge transformation, however they do yield new transition functions for a sequence of bundles. While it will be easy to see that these bundles will all be isomorphic to each other and the original bundle, the obvious changes to the gauge transformations mess with the uniform bounds, and thus 
the patching lemma will be needed in order to find less straightforward but more suitable isomorphisms between the bundles, which then can be modified in a uniform way to yield globally defined uniformly bounded gauge transformations. To get uniform bounds on the gauge transformations, we will also need the following.

Lemma 3.7 (cf. lemmata 1.33 and 1.34). Bounds on connection forms give bounds on the gauge transformations relating them, and moreover if two sequences of connections are uniformly bounded, then the sequence of gauge transformations relating them has a $C^{0}$ convergent subsequence.

Proof of weak compactness theorem. Let $\left(A^{i}\right)_{i \in \mathbb{N}} \subseteq \mathcal{A}^{1, p}(P)$ be a sequence such that $\left\|F_{A^{i}}\right\|_{p}$ is uniformly bounded. Choose $q<p$ such that it satisfies the hypotheses of the gauge fixing lemma 2.2 . We can bound the $L^{q}$-energy

$$
\mathcal{E}_{q}\left(\left.A^{i}\right|_{U}\right)=\left\|F_{A^{i}}\right\|_{q(U)}^{q} \stackrel{(1)}{\leq}(\operatorname{Vol} U)^{\frac{p-q}{p}}\left\|F_{A^{i}}\right\|_{p(U)}^{q} \leq(\operatorname{Vol} U)^{1-\frac{q}{p}}\left\|F_{A^{i}}\right\|_{p}^{q},
$$

where (1) is the Hölder inequality for $\frac{1}{q}=\frac{1}{p}+\frac{p-q}{p q}$. With this expression we can make $\mathcal{E}_{q}\left(\left.A^{i}\right|_{U}\right) \leq \tilde{\varepsilon}$ for $U$ of sufficiently small volume, and this is why it is important that $q<p$ strictly since we need $1-\frac{q}{p}>0$ to make the $L^{q}$-energy small. Now we are in the setting of 2.2 , and all the $A^{i}$ may be put in Uhlenbeck gauge on open sets which cover $M$; since $M$ is compact, we can take a finite subcollection $M=\bigcup_{\alpha=1}^{N} U_{\alpha}$.

These $U_{\alpha}$ form a bundle atlas for $P \rightarrow M$, and on each $U_{\alpha}$ the connections are represented by connection matrices $A_{\alpha}^{i} \in \mathcal{A}^{1, p}\left(U_{\alpha}\right)$. Since $\mathcal{E}_{q}\left(A_{\alpha}^{i}\right)=\mathcal{E}_{q}\left(\left.A^{i}\right|_{U_{\alpha}}\right) \leq \tilde{\varepsilon}$, there exist $u_{\alpha}^{i} \in \mathcal{G}^{2, p}\left(U_{\alpha}\right)$ such that $u_{\alpha}^{i *} A_{\alpha}^{i}$ is in Uhlenbeck gauge, and in particular $\left\|u_{\alpha}^{i *} A_{\alpha}^{i}\right\|_{1, p} \leq$ $\tilde{C}\left\|F_{A_{\alpha}^{i}}\right\|_{p}$ is uniformly bounded. This is sufficient to find weakly convergent subsequences on each $U_{\alpha}$, however the $u_{\alpha}$ do not necessarily define a global gauge transformation. For that to be the case we need

$$
u_{\alpha \beta}^{i}:=\left(u_{\alpha}^{i}\right)^{-1} \phi_{\alpha \beta} u_{\beta}^{i}
$$

to be identical to $\phi_{\alpha \beta}$, the transition functions of the bundle atlas, see 1.18 and the discussion in subsection $\left.1.2 .1\right|^{2}$ Therefore, the next step in the proof is modifying the $u_{\alpha}^{i}$ appropriately to achieve this.

In order to use the patching lemma 3.10 we need the transition functions to be $C^{0}$-close to each other. To see this, write

$$
\begin{aligned}
u_{\alpha \beta}^{i *}\left(u_{\alpha}^{i *} A_{\alpha}^{i}\right) & =\left(u_{\alpha}^{i} u_{\alpha \beta}^{i}\right)^{*} A_{\alpha}^{i}=\left(\phi_{\alpha \beta} u_{\beta}^{i}\right)^{*} A_{\alpha}^{i}=u_{\beta}^{i *}\left(\phi_{\alpha \beta}^{*} A_{\alpha}^{i}\right) \\
& =u_{\beta}^{i *} A_{\beta}^{i},
\end{aligned}
$$

and since all $u_{\alpha}^{i *} A_{\alpha}^{i}$ are uniformly bounded for any $\alpha \in A$, lemma 1.33 tells us that the gauge transformations relating these connection forms, $u_{\alpha \beta}^{i}$, are also uniformly bounded, and furthermore there is a subsequence of the $u_{\alpha \beta}^{i}$ (also labelled $i \in \mathbb{N}$ ) that converges in $C^{0}$. Thus, for a further subsequence, and for each $\alpha, \beta=1, \ldots, N$, all the $u_{\alpha \beta}^{i}$ can be made to be within a geodesic $\delta$-ball of each other for any $\delta>0$; in particular we can single out the first element of this subsequence $g_{\alpha \beta}:=u_{\alpha \beta}^{1}$ and denote also $g_{\alpha}:=u_{\alpha}^{1}$, and we have 3

$$
\mathrm{d}\left(u_{\alpha \beta}^{i}, g_{\alpha \beta}\right) \leq \delta,
$$

\footnotetext{
${ }^{2}$ Note that if we look at the gauge transformations $u_{\alpha}^{i}$ as local changes of trivialization, then the $u_{\alpha \beta}^{i}$ are new transition functions for an isomorphic bundle for each $i \leq N$; even so, this is not enough.

${ }^{3}$ See subsection 1.2 .2 for the definitions of a geodesic convex ball and this metric.

${ }^{4}$ Also note that the first element $g_{\alpha \beta}$ can only be fixed after the choice of $\delta$.
} 
Picking $\delta=\Delta_{\text {exp }}$ the radius of a convex geodesic ball on $G$, the patching lemma 3.10 gives us a refinement $V_{\alpha} \subseteq U_{\alpha}$ of the original cover and new gauge transformations $h_{\alpha}^{i}: V_{\alpha} \rightarrow G$ with uniform bounds and such that

$$
\left(h_{\alpha}^{i}\right)^{-1} u_{\alpha \beta}^{i} h_{\beta}^{i}=g_{\alpha \beta} .
$$

With these new gauge transformations we can now modify the original $u_{\alpha}^{i}$. Define $\tilde{u}_{\alpha}^{i}:=$ $u_{\alpha}^{i} h_{\alpha}^{i} g_{\alpha}$ on $V_{\alpha}$, and note that this defines a global gauge transformation, as $M=\bigcup_{\alpha=1}^{N} V_{\alpha}$ and on $V_{\alpha} \cap V_{\beta}$

$$
\left(\tilde{u}_{\alpha}^{i}\right)^{-1} \phi_{\alpha \beta} \tilde{u}_{\beta}^{i}=g_{\alpha}\left(h_{\alpha}^{i}\right)^{-1} \underbrace{\left(u_{\alpha}^{i}\right)^{-1} \phi_{\alpha \beta} u_{\beta}^{i}}_{u_{\alpha \beta}^{i}} h_{\beta}^{i} g_{\beta}^{-1}=g_{\alpha} g_{\alpha \beta} g_{\beta}^{-1}=\phi_{\alpha \beta} .
$$

Moreover, $\tilde{u}^{i}$ as defined by the local $\tilde{u}_{\alpha}^{i}$ is in $\mathcal{G}^{2, p}(P)$, as lemma 3.10 yields $h_{\alpha}^{i} \in \mathcal{G}^{2, p}\left(V_{\alpha}\right)$, we had $u_{\alpha}^{i} \in \mathcal{G}^{2, p}\left(V_{\alpha}\right)$ from the start and $\mathcal{G}^{k, p}$ is closed under group multiplication for $k p>n$, which is the case since $p>\frac{n}{2}$.

It remains to prove that $\tilde{u}_{\alpha}^{i *} A_{\alpha}^{i}$ is uniformly bounded in $\mathcal{A}^{1, p}\left(V_{\alpha}\right)$ for all $\alpha=1, \ldots, N$. This follows easily from lemma 1.31 , which in this case states that for $A^{i} \in \mathcal{A}^{1, p}(V)$ and $u^{i} \in \mathcal{G}^{2, p}(V)$ for some trivializing neighbourhood $V$, the following holds 5

$$
\left\|u^{i *} A^{i}\right\|_{1, p} \leq\left\|\left(u^{i}\right)^{-1} \mathrm{~d} u^{i}\right\|_{1, p}+c\left\|A^{i}\right\|\left(1+C_{W}\left\|\left(u^{i}\right)^{-1} \mathrm{~d} u^{i}\right\|_{1, p}\right),
$$

where $c, C_{W}$ are constants. Then writing

$$
\tilde{u}_{\alpha}^{i *} A_{\alpha}^{i}=\left(g_{\alpha}^{-1}\right)^{*} h_{\alpha}^{i *} u_{\alpha}^{i *} A_{\alpha}^{i},
$$

we first note that $h_{\alpha}^{i *}\left(u_{\alpha}^{i *} A_{\alpha}^{i}\right)$ is uniformly bounded: $\left\|u_{\alpha}^{i *} A_{\alpha}^{i}\right\|_{1, p}$ is bounded uniformly by the uniform bound on the curvature (because of the Uhlenbeck gauge), and $\left\|\left(h_{\alpha}^{i}\right) \mathrm{d} h_{\alpha}^{i}\right\|_{1, p}$ is uniformly bounded by the patching lemma and the fact that $\left\|\left(u_{\alpha \beta}^{i}\right)^{-1} \mathrm{~d} u_{\alpha \beta}^{i}\right\|_{1, p}$ has a uniform bound (lemma 1.33). Using (3.4) again, the uniform bound on $h_{\alpha}^{i *} u_{\alpha}^{i *} A_{\alpha}^{i}$ and the fact that $g_{\alpha}^{-1}$ is independent of $i \in \mathbb{N}$, we get a uniform $W^{1, p}$-bound on $\tilde{u}_{\alpha}^{i *} A_{\alpha}^{i}$.

Finally, we can use the Banach-Alaoglu theorem 1.18 to guarantee that for every $\alpha=$ $1, \ldots, N$, the sequence $\tilde{u}_{\alpha}^{i *} A_{\alpha}^{i}$ has a $W^{1, p}$-weakly convergent subsequence, and because the $\tilde{u}^{i}$ are global gauges, we can choose the same ${ }^{6}$ subsequence for all (finite) $\alpha$, which finally gives us a weakly convergent subsequence of $\tilde{u}^{i *} A^{i}$ in $\mathcal{A}^{1, p}(P)$.

Remark 3.8. Note that the choice of $\tilde{u}_{\alpha}^{i}$ makes sense. In finding a global gauge, the simplest thing to do would be to take $\tilde{u}_{\alpha}^{i}=\mathbb{1}_{G}$, and in order to keep the original $u_{\alpha}^{i}$ around for the Uhlenbeck gauge, it would have sufficed to have $\tilde{u}_{\alpha}^{i}=\mathbb{1}_{G}=u_{\alpha}^{i}\left(u_{\alpha}^{i}\right)^{-1}$; however, there is no uniform bound on $\left(u_{\alpha}^{i}\right)^{-1}$. In the patching lemma, it is already clear that $h_{\alpha}^{i}=\left(u_{\alpha}^{i}\right)^{-1} g_{\alpha}$ would have sufficed for the isomorphism, and if this could be guaranteed to have a uniform bound in this form then one could naturally write

$$
\tilde{u}_{\alpha}^{i}=\mathbb{1}_{G}=u_{\alpha}^{i} \underbrace{\left(u_{\alpha}^{i}\right)^{-1} g_{\alpha}}_{h_{\alpha}^{i}} g_{\alpha}^{-1} .
$$

\footnotetext{
${ }^{5}$ We used the Sobolev embedding $W^{1, p} \hookrightarrow L^{2 p}$, note that $1-\frac{n}{p} \geq-\frac{n}{2 p}$.

${ }^{6}$ For $\alpha=1$, there is a convergent subsequence; this subsequence is also uniformly bounded on $\alpha=2$, and so some further subsequence converges. By repeating this process until you find a convergent subsequence for $\alpha=N$, this same subsequence can be used for all $\alpha$. If there were countably many $\alpha$, a similar argument would work by taking the diagonal of the subsequences.
} 
Thus it makes sense to simply substitute the more complicated $h_{\alpha}^{i}$ found in the proof of the patching lemma in the expression above when choosing a uniformly bounded globally defined gauge transformation.

\subsection{Strong compactness}

While the strong compactness theorem is attributed to Uhlenbeck, it is not stated it in any of her 1982 papers. However, it is a simple corollary from the proof of the weak compactness theorem and a result on regularity of Yang-Mills connections in Coulomb gauge, which is a consequence of the gauge fixing lemma and she also stated in her paper [Uhl82. We show the adaptation of the proof, sketching the points which are already explained in the proof of theorem $3.57^{7}$

Theorem 3.9 (Strong compactness). Let $M$ be a compact Riemannian n-manifold with (possibly empty) boundary, and let $1<p<\infty$ such that $p>\frac{n}{2}$ and in case $n=2, p \geq \frac{4}{3}$. Suppose a sequence of connections $\left(A^{i}\right)_{i \in \mathbb{N}} \subseteq \mathcal{A}^{1, p}(P)$ is such that the $A^{i}$ are weak YangMills connections and $\left\|F_{A^{i}}\right\|_{p}$ is uniformly bounded. Then there exists a subsequence (with same label $i \in \mathbb{N}$ ) and a sequence of gauge transformations $\left(u^{i}\right)_{i \in \mathbb{N}} \subseteq \mathcal{G}^{2, p}(P)$ such that $u^{i *} A^{i}$ converges strongly with all derivatives to a smooth Yang-Mills connection.

Proof. Let $A^{i} \in \mathcal{A}^{1, p}(P)$ be as in the statement of the theorem. Choose $q$ to satisfy the hypotheses of the gauge fixing lemma, then there is a finite cover $M=\bigcup_{\alpha=1}^{N} U_{\alpha}$ such that $\mathcal{E}_{q}\left(A_{\alpha}^{i}\right) \leq \tilde{\varepsilon}$, and therefore there exist sequences of gauge transformations $\left(u_{\alpha}^{i}\right)_{i \in \mathbb{N}} \subseteq \mathcal{G}^{2, p}\left(U_{\alpha}\right)$ on each $U_{\alpha}$ such that from the uniform bound on $\left\|F_{A^{i}}\right\|_{p}$ we get a uniform bound on $\left\|u_{\alpha}^{i *} A_{\alpha}^{i}\right\|_{1, p}$ for each $\alpha$. Since the $A^{i}$ are weak Yang-Mills connections, then so are the $u_{\alpha}^{i *} A_{\alpha}^{i}$ (lemma 1.36), and therefore from the regularity theorem 1.39 we find uniform bounds on $\left\|u_{\alpha}^{i *} A_{\alpha}^{i}\right\|_{k, p}$ for all $k \in \mathbb{N}$.

Once again we look at the transition functions $u_{\alpha \beta}^{i}$ as defined in (3.1), where the $\phi_{\alpha \beta}$ are the transition functions for our bundle. From $(3.2)$ and lemma 1.34 we see $u_{\alpha \beta}^{i} \in \mathcal{G}^{k, p}$ for all $k$, and then from lemma 1.33 we find uniform bounds on $\left\|\left(u_{\alpha \beta}^{i}\right)^{-1} \mathrm{~d} u_{\alpha \beta}^{i}\right\|_{k, p}$ for all $k$, and a subsequence of the $u_{\alpha \beta}^{i}$ that converges in $C^{0}$ which can be taken the same subsequence for all $\alpha, \beta=1, \ldots, N$. Therefore there is some $i$ such that all the $u_{\alpha \beta}^{i}$ are within a $\Delta_{\exp }$ sized $C^{0}$-ball, for $\Delta_{\exp }$ the radius of a convex geodesic ball on $G$, and we take this $i$ to be the first element of the sequence. Now, instead of fixing $u_{\alpha}^{1}$ as we did for the proof of 3.5 , we will need to take smooth $g_{\alpha} \in \mathcal{G}\left(U_{\alpha}\right)$ that are $C^{0}$-close to $u_{\alpha}^{1}$ for each $\alpha$, which will imply that $g_{\alpha \beta}:=g_{\alpha}^{-1} \phi_{\alpha \beta} g_{\beta}$ will also be $C^{0}$-close to the $u_{\alpha \beta}^{i}$.

We apply the patching lemma 3.10 to find a refinement $V_{\alpha} \subseteq U_{\alpha}$ of the original cover and $h_{\alpha}^{i} \in \mathcal{G}^{k, p}\left(V_{\alpha}\right)$ such that

$$
\left(h_{\alpha}^{i}\right)^{-1} u_{\alpha \beta}^{i} h_{\beta}^{i}=g_{\alpha \beta}
$$

on $V_{\alpha} \cap V_{\beta}$ and such that there are uniform bounds on $\left\|\left(h_{\alpha}^{i}\right)^{-1} \mathrm{~d} h_{\alpha}^{i}\right\|_{k, p\left(V_{\alpha}\right)}$ for all $k \in \mathbb{N}$. Then $\tilde{u}_{\alpha}^{i}:=u_{\alpha}^{i} h_{\alpha}^{i} g_{\alpha}^{-1}$ will patch to global gauge transformations as in 3.3 , and the inequality in lemma 1.31 will take the form 8

$$
\left\|u^{i *} A^{i}\right\|_{k, p} \leq\left\|\left(u^{i}\right)^{-1} \mathrm{~d} u^{i}\right\|_{k, p}+c\left\|A^{i}\right\|\left(1+C_{W}\left\|\left(u^{i}\right)^{-1} \mathrm{~d} u^{i}\right\|_{k, p}\right)^{k},
$$

\footnotetext{
${ }^{7}$ Throughout this proof we once again keep taking subsequences and relabelling them the same as the original sequence, with $i \in \mathbb{N}$.

${ }^{8} W^{k-1,2 p} \hookrightarrow W^{k, p}$ since $p>\frac{n}{2}$.
} 
slightly more complicated than (3.4), which we use to find uniform $W^{k, p}$-bounds on $\tilde{u}_{\alpha}^{i *} A_{\alpha}^{i}=$ $\left(g_{\alpha}^{-1}\right)^{*} h_{\alpha}^{i *} u_{\alpha}^{i *} A_{\alpha}^{i}$ for all $k \in \mathbb{N}$, on each $V_{\alpha}$. Here it is important that the $g_{\alpha}^{i}$ are smooth in order to preserve the bounds for all $k$.

Finally, having obtained uniform bounds on the $W^{k, p}$-norms of the local gauged connections for all $k \in \mathbb{N}$, we may use the Arzelà-Ascoli theorem to find subsequences which converge uniformly with all derivatives, and then take the same subsequence on all $V_{\alpha}$ such that the $\tilde{u}_{\alpha}^{i}$ patch to a global $\tilde{u}^{i}$ and $\tilde{u}^{i *} A^{i}$ converges uniformly with all derivatives to some smooth connection $\tilde{A} \in \mathcal{A}(P)$. Then, from lemmata 1.36 and $1.38, \tilde{u}^{i} A^{i}$ is weak Yang-Mills for each $i \in \mathbb{N}$ and the limit connection $\tilde{A}$ will also be weak Yang-Mills; since it is smooth, it is a Yang-Mills connection.

In the book Weh04 another approach is used to prove the strong compactness theorem, due to Dietmar Salamon. Rather than adapt the proof of the weak compactness theorem, relies on a local slice theorem and then applies theorem 3.5 directly. Observe, however, that it needs strict inequality $p>\frac{4}{3}$ in case $n=2$ because there will only be weak convergence of the connections, see lemma 1.38 . ( $i i)$.

\subsection{Patching}

Finally, we prove the patching lemma used in the proofs of the weak and strong compactness theorems.

Lemma 3.10 (Patching lemma). Let $M$ be an n-manifold, $p>\frac{n}{2}$ and let $M=\bigcup_{\alpha \in \mathbb{N}} U_{\alpha}$ be a locally finite open cover by precompact 9 sets. Then there is a refinement $V_{\alpha} \subseteq U_{\alpha}$ such that the following holds:

(i) Let $k \in \mathbb{N}$ and let $g_{\alpha \beta}, h_{\alpha \beta} \in \mathcal{G}^{k+1, p}\left(U_{\alpha} \cap U_{\beta}\right)$ be two sets of transition functions for some principal $G$-bundle over $M$ such that

$$
\mathrm{d}\left(g_{\alpha \beta}, h_{\alpha \beta}\right) \leq \Delta_{\exp }, \quad \forall \alpha, \beta \in \mathbb{N} .
$$

Then there exist gauge transformations $h_{\alpha} \in \mathcal{G}^{k+1, p}\left(V_{\alpha}\right)$ for all $\alpha \in \mathbb{N}$ such that on all intersections $V_{\alpha} \cap V_{\beta}$

$$
h_{\alpha}^{-1} h_{\alpha \beta} h_{\beta}=g_{\alpha \beta} .
$$

(ii) Let $K \geq 2$ be an integer or $K=\infty$. If the $h_{\alpha \beta}$ in $(i)$ run through a sequence $h_{\alpha \beta}^{i}$ of sets of transition functions such that $h_{\alpha \beta}^{i}, g_{\alpha \beta} \in \mathcal{G}^{k+1, p}\left(U_{\alpha} \cap U_{\beta}\right)$ for all $k<K$, and moreover for all $\alpha, \beta \in \mathbb{N}$ and $k<K$ there is a uniform bound on

$$
\left\|\left(h_{\alpha \beta}^{i}\right)^{-1} \mathrm{~d} h_{\alpha \beta}^{i}\right\|_{k, p\left(U_{\alpha} \cap U_{\beta}\right)} .
$$

Then the gauge transformations $h_{\alpha}^{i}$ in $(i)$ satisfy, for all $\alpha \in \mathbb{N}$ and $k<K, h_{\alpha}^{i} \in$ $\mathcal{G}^{k+1}\left(V_{\alpha}\right)$ and

$$
\sup _{i \in \mathbb{N}}\left\|\left(h_{\alpha}^{i}\right)^{-1} \mathrm{~d} h_{\alpha}^{i}\right\|_{k, p\left(V_{\alpha}\right)}<\infty
$$

which is to say that these norms are uniformly bounded for each $\alpha$ and $k$.

\footnotetext{
${ }^{9}$ Closure is compact.
} 
Proof. Note that we assume the cover is countable. We will first prove $(i)$ by induction on the cover, and then for a sequence of the constructed $h_{\alpha}^{i}$ constructed in $(i)$, regularity will follow directly and the uniform bounds will be proved by another induction on the cover.

For $(i)$, on each step $j \in \mathbb{N}$, we will construct $h_{j}$ on $V_{j}:=U_{j}$, while changing some (or none) of the previous $V_{\alpha}$ already constructed for $\alpha \leq j-1$ and keeping $h_{\alpha}$ the same, albeit with possibly smaller domain. For each $V_{\alpha}$, the process will end in finitely many steps, because as will be seen during the construction, on the $j$-th step a certain $V_{\alpha}$ will only be modified if $V_{\alpha} \cap U_{j} \neq \varnothing$, and the cover is locally finite. Moreover, the $V_{\alpha}$ will not depend on $k \in \mathbb{N}$ (from the Sobolev exponent) or the transition functions $h_{\alpha \beta}, g_{\alpha \beta}$. For the $h_{j}$ constructed, we will want three conditions to be satisfied at each step $j$ :

(1) the one we are trying to achieve, that is, condition (3.6),

$$
h_{\alpha}^{-1} h_{\alpha \beta} h_{\beta}=g_{\alpha \beta},
$$

on $V_{\alpha} \cap V_{\beta}$ for all $\alpha, \beta \leq j$,

(2) a technical condition which will be important within the construction,

$$
\mathrm{d}\left(h_{i \alpha} h_{\alpha} g_{\alpha i}, \mathbb{1}\right) \leq \Delta_{\exp },
$$

on $V_{\alpha} \cap U_{i}$ for all $\alpha \leq j$ and $i \geq j$,

(3) and regularity, $h_{\alpha} \in \mathcal{G}^{k+1}\left(V_{\alpha}\right)$ for all $\alpha \leq j$.

For the first step, $j=1$, we take $V_{1}:=U_{1}$ and $h_{1}:=\mathbb{1}$. Conditions (1) and (3) are trivially satisfied ${ }^{10}$ and for all $i \geq 1, \mathrm{~d}\left(h_{i 1} h_{1} g_{1 i}, \mathbb{1}\right)=\mathrm{d}\left(g_{1 i}, h_{1 i}\right) \leq \Delta_{\exp }$ on $V_{1} \cap U_{i}$ by assumption, therefore condition (2) is also satisfied.

At an arbitrary $j$-th step, for the induction hypothesis we will assume that for all $\alpha \leq j-1$ we have constructed $V_{\alpha} \subseteq U_{\alpha}$ and $h_{\alpha}$ such that there is still an open cover of $M$ and the $h_{\alpha}$ respect conditions (1)-(3). That is, $M=\bigcup_{\alpha<j} V_{\alpha} \cup \bigcup_{\alpha \geq j} U_{\alpha}$, and conditions (1)-(3) were met on the $(j-1)$-th step.

Then, for $\alpha=j$, we will take $V_{j}:=U_{j}$ and construct $h_{j}$ such that (1)-(3) are satisfied. We will see that there is a natural choice of $h_{j}$ on the intersection with the $V_{\alpha}$ which will automatically yield condition (1). We could then take $h_{j}=\mathbb{1}$ on $U_{j} \backslash \bigcup_{\alpha<j} V_{\alpha}$ and be done, but then $h_{j}$ might not even be continuous, let alone in $\mathcal{G}^{k+1}\left(U_{j}\right)$. The idea, then, is to write $h_{j}:=\exp \left(\psi_{j} \xi_{j}\right)$ for some $\xi_{j} \in \mathfrak{g}$ and a cutoff function $\psi_{j}$. Condition (2) is what is needed to have a well defined $\xi_{j}$, and the $V_{\alpha}$ will be modified so that there can be two disjoint compact sets where $\psi_{j}$ will take the values 0 and 1 .

Consider $\rho_{j}: U_{j} \cap \bigcup_{\alpha<j} V_{\alpha} \rightarrow G$ given by $\rho_{j}:=h_{j \alpha} h_{\alpha} g_{\alpha j}$ on $U_{j} \cap V_{\alpha}$. This is well defined, since (3.6) is satisfied for $\alpha, \beta<j$ by assumption (condition (1)) and so on intersections $U_{j} \cap V_{\alpha} \cap V_{\beta}$

$$
h_{j \beta} h_{\beta} g_{\beta j}=\left(h_{j \alpha} h_{\alpha \beta}\right) h_{\beta}\left(g_{\beta \alpha} g_{\alpha j}\right)=h_{j \alpha} h_{\alpha} g_{\alpha j} .
$$

This is exactly what we need $h_{j}$ to be on the intersections $U_{j} \cap V_{\alpha}$, as

$$
h_{\alpha}^{-1} h_{\alpha j}\left(h_{j \alpha} h_{\alpha} g_{\alpha j}\right)=g_{\alpha j} .
$$

As a product of $\mathcal{G}^{k+1, p}$ maps ${ }^{11} \rho \in \mathcal{G}^{k+1, p}\left(U_{j} \cap \bigcup_{\alpha<j} V_{\alpha}\right)$, and moreover condition (2) for $\alpha \leq j-1$ guarantees that $\mathrm{d}(\rho, \mathbb{1}) \leq \Delta_{\text {exp }}$, and therefore $\rho$ takes values in the convex 

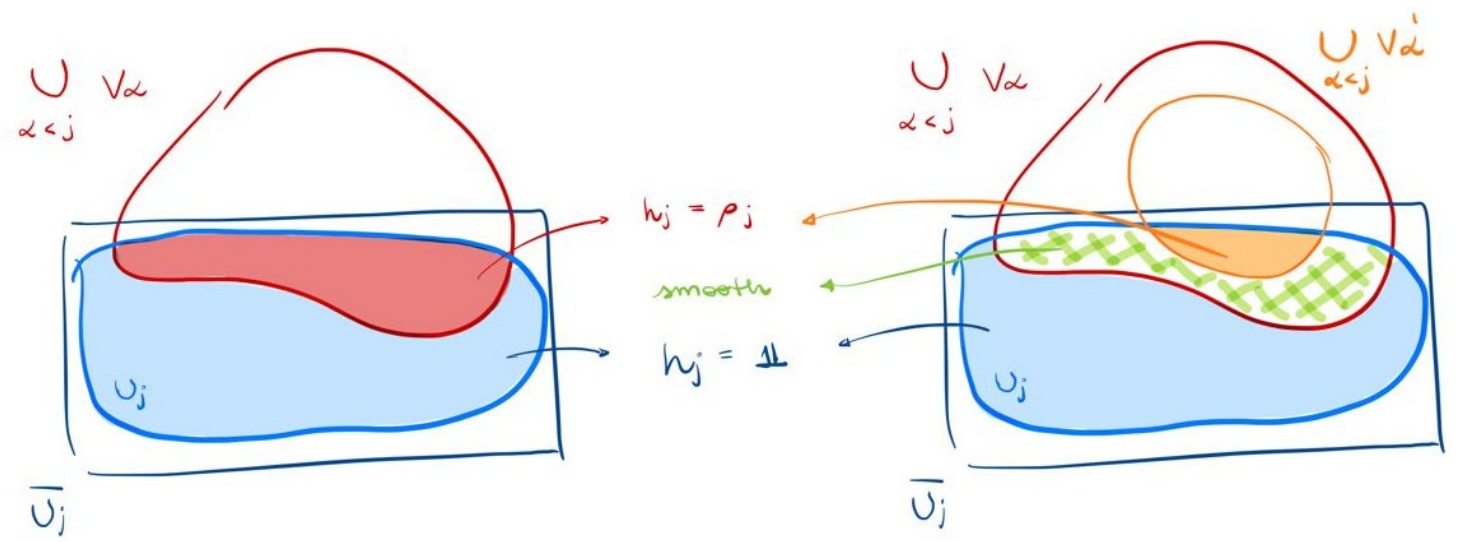

Figure 3.1: A sketch of the involved sets on the $j$-th step, illustrating the reason to make the $V_{\alpha}$ into smaller $V_{\alpha}^{\prime}$ : so that the $h_{j}$ will be appropriately smooth.

geodesic ball $B_{\Delta_{\exp }}(\mathbb{1})$. Therefore, there exists $\xi_{j}: W^{k+1, p}\left(U_{j} \cap \bigcup_{\alpha<j} V_{\alpha}\right) \rightarrow \mathfrak{g}$ such that $\rho_{j}=\exp \left(\xi_{j}\right)$.

Next, we replace the $V_{\alpha}, \alpha<j$, by possible smaller $V_{\alpha}^{\prime} \subseteq V_{\alpha}$, so that we can take (see Fig. 3.3

$$
h_{j}= \begin{cases}\rho_{j}=\exp \left(\xi_{j}\right) & \text { on } U_{j} \cap \bigcup_{\alpha<j} V_{\alpha}^{\prime}, \\ \mathbb{1}=\exp (0) & \text { on } U_{j} \backslash \bigcup_{\alpha<j} V_{\alpha}\end{cases}
$$

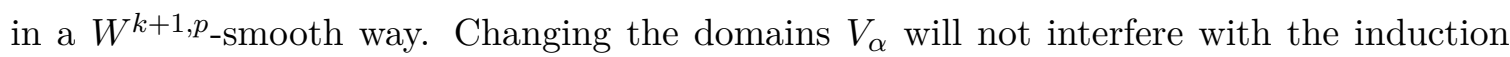
hypothesis, as conditions (1)-(3) remain valid when diminishing the domain of the $h_{\alpha}$, so long as we are still left with an open cover of $M$.

$B:=\overline{U_{j}} \backslash \bigcup_{\alpha<j} V_{\alpha}$ is compact and is where $h_{j}=\mathbb{1}$. We need to make it disjoint to $C:=\overline{U_{j} \cap \bigcup_{\alpha<j} V_{\alpha}^{\prime}}$, where we will want $h_{j}=\rho_{j}$. Let $N=M \backslash \bigcup_{\alpha \geq j} U_{\alpha} \subseteq \bigcup_{\alpha<j} U_{\alpha}$. Note that it is closed and covered by finitely many precompact sets, and therefore it is compact. For $\ell=1, \ldots, j-1$, we will change one $V_{\ell}$ at a time. Define

$$
A_{\ell}:=N \backslash\left(\bigcup_{\alpha<\ell} V_{\alpha}^{\prime} \cup \underset{\ell<\alpha<j}{\bigcup} V_{\alpha}\right) .
$$

The $A_{\ell}$ are compact and

$$
A_{\ell} \subseteq V_{\ell} \subseteq B^{c} .
$$

Then there exists an open set $V_{\ell}^{\prime} \subseteq V_{\ell}$ such that

$$
A_{\ell} \subseteq V_{\ell}^{\prime} \subseteq \overline{V_{\ell}^{\prime}} \subseteq B^{c}
$$

The cover is preserved at each step since $A_{\ell} \subseteq V_{\ell}^{\prime}$ and the $A_{\ell}$ are chosen such that they cover whatever parts of $N$ the other $V_{\alpha}$ and $V_{\alpha}^{\prime}$ do not, then

$$
M=N \cup \bigcup_{\alpha>j} U_{\alpha} \text { and } N \subseteq \bigcup_{\alpha<\ell} V_{\alpha}^{\prime} \cup A_{\ell} \cup \bigcup_{\ell<\alpha<j} V_{\alpha} \text {. }
$$

\footnotetext{
${ }^{10}$ Remember $h_{\alpha \alpha}=g_{\alpha \alpha}=\mathbb{1}$.

${ }^{11} \mathrm{By}$ assumption for the transition functions and by the induction hypothesis for the $h_{\alpha}$.
} 


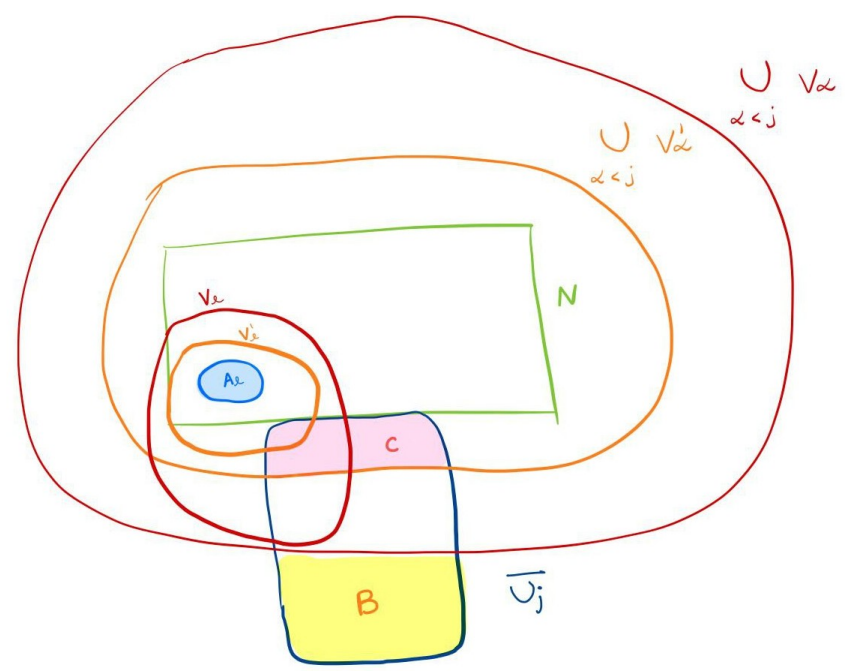

Figure 3.2: A sketch of the construction of the sets $B$ and $C$, that we need to make disjoint.

Also, $C \subseteq \bigcup_{\alpha<j} \overline{V_{\alpha}^{\prime}} \subseteq B^{c}$, and therefore $B \cap C=\varnothing$. If $V_{\ell} \cap U_{j}=\varnothing$, also $V_{\ell} \cap \overline{U_{j}}=\varnothing$ and so $V_{\ell}$ makes no difference for the definition of $C$ and does not need to be changed, $V_{\ell}^{\prime}:=V_{\ell}$, making the process finite for each $\alpha \in \mathbb{N}$.

We can now say that there exists a cutoff function $\psi_{j}: \overline{U_{j}} \rightarrow[0,1]$ such that $\psi(B)=0$ and $\psi(C)=1.12$ Then let

$$
h_{j}:= \begin{cases}\exp \left(\psi_{j} \xi_{j}\right) & \text { on } V_{j} \cap \bigcup_{\alpha<j} V_{\alpha} \\ \mathbb{1} & \text { on } V_{j} \backslash \bigcup_{\alpha<j} V_{\alpha}\end{cases}
$$

Note that, for $x \in V_{j} \cap V_{\alpha}, h_{j}(x)=\exp (a \xi)=\phi_{\xi}^{t=a}(\mathbb{1})$ for some $a \in[0,1]$ and $\xi \in \mathfrak{g}$, and thus $h_{j}(x)$ is part of some geodesic between $\mathbb{1}$ and $\exp (\xi)=\exp \left(\xi_{j}(x)\right)=\rho_{j}(x)$. Then

$$
h_{j}(x) \begin{cases}=h_{j \alpha} h_{\alpha} g_{\alpha j}(x) & \text { on } V_{j} \cap V_{\alpha}^{\prime}, \text { for } \alpha \leq j-1, \\ \in \gamma\left(\mathbb{1},\left(h_{j \alpha} h_{\alpha} g_{\alpha j}\right)(x)\right) & \text { on } V_{j} \cap V_{\alpha}, \text { for } \alpha \leq j-1 \\ \equiv \mathbb{1} & \text { on } V_{j} \backslash \bigcup_{\alpha<j} V_{\alpha} .\end{cases}
$$

Now, we check that $h_{j}$ satisfies conditions (1)-(3). The first equality shows that condition (1) holds now for $\alpha, \beta \leq j$ when replacing $V_{\alpha}$ by $V_{\alpha}^{\prime}$, as we had already seen with (3.9). Condition (2) remains valid for $\alpha \leq j-1$ from the induction hypothesis with $V_{\alpha}^{\prime} \subseteq V_{\alpha}$, and we now check that for $i \geq j+1$ it is valid on $V_{j} \cap U_{i}$. On $V_{j} \cap U_{i} \backslash \bigcup_{\alpha<j} V_{\alpha}$, we have $h_{j} \equiv \mathbb{1}$, and so

$$
\mathrm{d}\left(h_{i j} h_{j} g_{j i}, \mathbb{1}\right)=\mathrm{d}\left(h_{i j} g_{j i}, \mathbb{1}\right)=\mathrm{d}\left(h_{i j}, g_{i j}\right) \leq \Delta_{\text {exp }},
$$

from the original assumption on the transition functions. On $V_{j} \cap U_{i} \cap V_{\alpha}$ for some $\alpha \leq j-1$, we show that $h_{j}$ lies on the convex geodesic ball $B_{\Delta_{\text {exp }}}\left(h_{j i} g_{i j}\right)$, and therefore

$$
\mathrm{d}\left(h_{i j} h_{j} g_{j i}, \mathbb{1}\right)=\mathrm{d}\left(h_{j}, h_{j i} g_{i j}\right) \leq \Delta_{\exp },
$$

as we wish. First, note that we have shown that $h_{j}$ lies on the unique minimal geodesic from $\mathbb{1}$ to $h_{j \alpha} h_{\alpha} g_{\alpha j}$. Now,

$$
\mathrm{d}\left(\mathbb{1}, h_{j i} g_{i j}\right)=\mathrm{d}\left(h_{i j}, g_{i j}\right) \leq \Delta_{\exp }
$$

\footnotetext{
${ }^{12}$ Take a partition of unity subordinate to the cover $B^{c}$ and $C^{c}$, for instance.
} 
by assumption on the transition functions, and

$$
\mathrm{d}\left(h_{j \alpha} h_{\alpha} g_{\alpha j}, h_{j i} g_{i j}\right)=\mathrm{d}\left(h_{\alpha}, h_{\alpha j} h_{j i} g_{i j} g_{j \alpha}\right)=\mathrm{d}\left(h_{\alpha}, h_{\alpha i} g_{i \alpha}\right)=\mathrm{d}\left(h_{i \alpha} h_{\alpha} g_{\alpha i}, \mathbb{1}\right) \leq \Delta_{\exp }
$$

by the induction hypothesis for $\alpha \leq j-1$. Both endpoints of $\gamma\left(\mathbb{1}, h_{j \alpha} h_{\alpha} g_{\alpha j}\right)$ lie in $B_{\Delta_{\exp }}\left(h_{j i} g_{i j}\right)$, and therefore the geodesic is entirely contained in the ball. Finally, condition (3) is still met by $h_{\alpha}$ for $\alpha \leq j-1$ because $h_{\alpha} \in \mathcal{G}^{k+1, p}\left(V_{\alpha}\right)$ and restricting to a smaller domain preserves the regularity; for $\alpha=j$, we know that $\psi_{j}$ is smooth and $\xi_{j} \in W^{k+1, p}\left(V_{j}, \mathfrak{g}\right)$, and therefore $h_{j}=\exp \left(\psi_{j} \xi_{j}\right) \in \mathcal{G}^{k+1, p}\left(V_{j}\right)$, by lemma 1.21 .

\section{We are done with the proof of $(i)$.}

For (ii), we have instead of $h_{\alpha \beta}$ a sequence of $h_{\alpha \beta}^{i}$, each of which is close to $g_{\alpha \beta}$, the transition functions $g_{\alpha \beta}, h_{\alpha \beta}^{i}$ are said to be in the appropriate Sobolev gauge group $\mathcal{G}^{k+1, p}$ for all $k<K$, and moreover there are uniform bounds on $\left\|\left(h_{\alpha \beta}^{i}\right)^{-1} \mathrm{~d} h_{\alpha \beta}^{i}\right\|_{k, p\left(U_{\alpha} \cap U_{\beta}\right)}$ for each $\alpha, \beta$. We need to check the regularity of the $h_{\alpha}^{i}$ for all $k<K$, and that there are uniform bounds on $\left\|\left(h_{\alpha}^{i}\right)^{-1} \mathrm{~d} h_{\alpha}^{i}\right\|_{k, p\left(V_{\alpha}\right)}$ for each $\alpha$.

Since the construction of each $h_{\alpha}^{i}$ in (i) is independent of the Sobolev exponent $k$ and regularity followed from the original regularity of the transition functions, we have $h_{\alpha}^{i} \in$ $\mathcal{G}^{k+1, p}\left(V_{\alpha}\right)$ for all $k<K$.

For each $k<K$, the uniform bounds will follow by another induction on the cover. The first step is trivial, since the construction in $(i)$ begins with $h_{1}^{i} \equiv \mathbb{1}$ and so $\mathrm{d} h_{1}^{i}=0$ and the norm is just zero. For the induction hypothesis we will assume that for all $\alpha \leq j-1$ there are uniform bounds on $\left\|\left(h_{\alpha}^{i}\right)^{-1} \mathrm{~d} h_{\alpha}^{i}\right\|_{k, p\left(U_{\alpha}\right)}$. Note that $h_{j}^{i}=\exp \left(\psi_{j} \xi_{j}^{i}\right)$ on $U_{j}$ and $\xi_{j}^{i}$ is defined by $\rho_{j}^{i}=\exp \left(\xi_{j}^{i}\right)=h_{j \alpha}^{i} h_{\alpha}^{i} g_{\alpha j}$ on $U_{j} \cap V_{\alpha}$ for $\alpha \leq j-1$. We will show that there are uniform bounds on $\rho_{j}^{i}$ on the intersections of $U_{j}$ with each $V_{\alpha}$, which will then give uniform bounds on $\xi_{j}^{i}$ on $U_{j} \cap \bigcup_{\alpha<j} V_{\alpha}$, and in turn this will give the uniform bounds on $h_{j}^{i}$.

For the uniform bound for $\rho_{j}^{i}$, first note the estimate for $u, v \in \mathcal{G}^{k+1, p}(U)$ over some precompact set $U$,

$$
\begin{aligned}
\left\|(u v)^{-1} \mathrm{~d}(u v)\right\|_{k, p} & =\left\|v^{-1} u^{-1}(u \mathrm{~d} v+\mathrm{d} u \cdot v)\right\|_{k, p} \\
& \leq\left\|v^{-1} \mathrm{~d} v\right\|_{k, p}+\left\|v^{-1}\left(u^{-1} \mathrm{~d} u\right) v\right\|_{k, p} \\
& \stackrel{(1)}{\leq}\left\|v^{-1} \mathrm{~d} v\right\|_{k, p}+C\left\|u^{-1} \mathrm{~d} u\right\|_{k, p}\left(1+\left\|v^{-1} \mathrm{~d} v\right\|_{k-1,2 p}\right)^{k} \\
& \stackrel{(2)}{\leq}\left\|v^{-1} \mathrm{~d} v\right\|_{k, p}+C\left\|u^{-1} \mathrm{~d} u\right\|_{k, p}\left(1+\left\|v^{-1} \mathrm{~d} v\right\|_{k, p}\right)^{k}
\end{aligned}
$$

where in (1) we used lemma 1.32 , and in (2) we used the Sobolev estimate for $W^{k-1,2 p} \hookrightarrow$ $W^{k, p}$, which is valid over $U$ even if $M$ is not compact. Since we have uniform bounds over $U_{j} \cap V_{\alpha}$ on $\left\|\left(h_{j \alpha}^{i}\right)^{-1} \mathrm{~d} h_{j \alpha}^{i}\right\|_{k, p}$ by assumption, on $\left\|\left(h_{\alpha}^{i}\right)^{-1} \mathrm{~d} h_{\alpha}^{i}\right\|_{k, p}$ by induction hypothesis and on $\left\|\left(g_{\alpha j}\right)^{-1} \mathrm{~d} g_{\alpha j}\right\|_{k, p}$ because it is independent of $i$, we can apply this estimate to $(u, v)=$ $\left(h_{j \alpha}^{i}, h_{\alpha}^{i}\right)$ and then to $(u, v)=\left(h_{j \alpha}^{i} h_{\alpha}^{i}, g_{\alpha j}\right)$ to get a uniform bound on $\left\|\left(\rho_{j}^{i}\right)^{-1} \mathrm{~d} \rho_{j}^{i}\right\|_{k, p\left(U_{j} \cap V_{\alpha}\right)}$. Because there are finitely many intersections $U_{j} \cap V_{\alpha}$, this gives an overall uniform bound for $\left\|\left(\rho_{j}^{i}\right)^{-1} \mathrm{~d} \rho_{j}^{i}\right\|_{k, p}$ on its whole domain of definition, $U_{j} \cap \bigcup_{\alpha<j} V_{\alpha}$.

Finally, we can use this to get a uniform bound for $\left\|\xi_{j}^{i}\right\|_{k, p}$ on $U_{j} \cap \bigcup_{\alpha<j} V_{\alpha}$ in a rather roundabout way, which we only sketch. First, fix an embedding $\Phi: B_{\Delta_{\exp }}(\mathbb{1}) \subseteq G \rightarrow \mathbb{R}^{2 d+1}$, 
which contains the image of every $\rho_{j}^{i}$, and use the bound on $\left(\rho_{j}^{i}\right)^{-1} \mathrm{~d} \rho_{j}^{i}$ to achieve bounds on the embedding; this will then give bounds on a global chart $\phi: B_{\Delta_{\exp }}(\mathbb{1}) \rightarrow \mathbb{R}^{d}$, and subsequently on $\xi_{j}^{i}$. To see this more clearly, make the following definitions: let $U:=U_{j} \cap$ $\bigcup_{\alpha<j} V_{\alpha}$, then let $\tilde{\phi}: \mathfrak{g} \rightarrow \mathbb{R}^{d}$ be an isomorphism, and use it to define a chart $\phi:=\tilde{\phi} \circ \exp ^{-1}$ and an embedding $\Phi:=(\phi, 0)=\iota \circ \phi$ for $\iota: \mathbb{R}^{d} \hookrightarrow \mathbb{R}^{2 d+1}$. To get from bounds on $\rho$ to bounds on the embedding, use these definitions and see the calculations on page 189 of [Weh04; then the calculations on page 187 give estimates on the chart, which gives estimates on $\xi_{j}^{i}$ via the isomorphism, and finally see the calculations on page 188 to achieve estimates on the $h_{j}^{i}$.

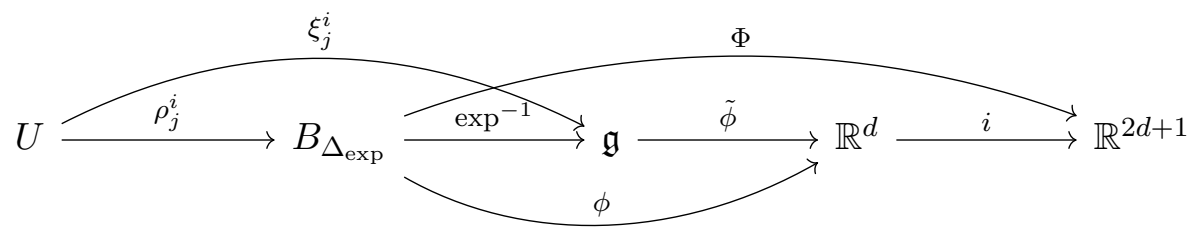

Figure 3.3: Embedding $\Phi$ and chart $\phi$ of $B_{\Delta_{\exp }} \subseteq G$. We have dropped the subscript $j$ and defined $U:=U_{j} \cap \bigcup_{\alpha<j} V_{\alpha}$. 


\section{Appendix A}

\section{BACKGROUND MATERIAL}

We will need some results on calculus of Banach spaces. A good source is [AP95].

Definition A.1. Let $X$ and $Y$ be Banach spaces, and $U \subseteq X$ an open subset, and consider a map $F: U \rightarrow Y$. We say that $F$ is Fréchet differentiable at $u \in U$ if there exists a bounded operator $A: X \rightarrow Y$ such that

$$
\lim _{\|h\| \rightarrow 0} \frac{\| F(u+h)-F(u)-A(h \|)}{\|h\|}=0 .
$$

Such an $A$ is uniquely determined as is called the (Fréchet) differential of $F$ at $u$.

The following result is the analogous to the usual mean value theorem of calculus.

Proposition A.2 (Mean value inequality). Let $F: U \rightarrow Y$ be differentiable. For $u, v \in U$ such that the line segment $[u, v]$ is contained in $U$,

$$
\|F(u)-F(v)\| \leq \sup _{w \in[u, v]}\|\mathrm{d} F(w)\| .
$$

And this is the implicit function theorem for Banach spaces.

Theorem A.3 (Implicit function theorem). Let $T: X \times Y \rightarrow Z$ be a continuous map between Banach spaces that is differentiable with respect to $Y$, and suppose there is a point $(\alpha, \beta) \in X \times Y$ such that $T(\alpha, \beta)=0$ and $\left.\partial_{Y} T\right|_{(\alpha, \beta)}$ is bijective.

Then there exist neighbourhoods $U \subseteq X$ and $V \subseteq Y$ of $\alpha$ and $\beta$, respectively, and a continuous map $f: U \rightarrow V$ such that for all $x \in U, T(x, f(x))=0$.

Lemma A.4. If $T$ is an operator on a Banach space such that $\|T-I\|<1$, then

$$
\left\|T^{-1}\right\| \leq \frac{1}{1-\|T-I\|}
$$

Lemma A.5 ([Weh04], lemma E.4). Let $T, S: X \rightarrow Z$ be bounded linear operators between Banach spaces, and suppose that $T$ is bijective and $\left\|T^{-1}\right\|\|S\|<1$. Then the perturbed operator $T+S$ is also bijective with bounded inverse. 


\section{The Neumann problem}

The first four chapters of Weh04 are fully devoted to giving a good and thorough introduction to the Neumann problem, beginning with the $L^{2}$ theory for the homogeneous problem, generalization to $L^{p}$-spaces and sections of vector bundles, and the inhomogeneous problem. We quote here only a few results that will be needed in chapter 2 For $M$ a compact manifold with boundary, the Neumann problem is as follows:

$$
\left\{\begin{array}{lc}
\Delta u=f & \text { on } M, \\
\frac{\partial u}{\partial \nu}=g & \text { on } \partial M,
\end{array}\right.
$$

where $\nu$ is the exterior normal direction. The problem is said homogeneous when $g=0$. From now on let $1<p<\infty$ and $k \in \mathbb{N}_{0}$. If $f \in W^{k, p}(M)$, then the natural space for the boundary values $g$ is

$$
W_{\partial}^{k+1, p}(M):=\frac{W^{1, p}(M)}{W_{\delta}^{k+1, p}(M)},
$$

where $W_{\delta}^{k+1, p}(M)$ is defined as the closure in $W^{k+1, p}(M)$ of the smooth functions vanishing on the boundary. The norm on this space is

$$
\|g\|_{W_{\partial}^{k+1, p}}=\inf \left\{\|G\|_{k+1, p}: G \in W^{k+1, p}(M) \text { and } g=\left.G\right|_{\partial M}\right\} .
$$

Theorem A.6 ([Weh04, theorem 3.1). Let $f \in L^{p}(M)$ and $g \in W_{\partial}^{1, p}(M)$. Then there exists a solution $u \in W^{2, p}(M)$ the Neumann problem if and only if

$$
\int_{M} f+\int_{\partial M} g=0
$$

The solution is unique up to an additive constant.

Proposition A.7 ([Weh04, theorem 3.2). There exist constants $C, C^{\prime}$ such that for all $u \in W^{k+2, p}(M)$,

$$
\begin{aligned}
& \|u\|_{k+2, p} \leq C^{\prime}\left(\|\Delta u\|_{k, p}+\left\|\frac{\partial u}{\partial \nu}\right\|_{W_{\partial}^{k+1, p}}+\|u\|_{k+1, p}\right), \\
& \|u\|_{k+2, p} \leq C\left(\|\Delta u\|_{k, p}+\left\|\frac{\partial u}{\partial \nu}\right\|_{W_{\partial}^{k+1, p}}\right), \quad \text { if } \int_{M} u=0 .
\end{aligned}
$$

Moreover, for each $k \in \mathbb{N}_{0}$, these constants depend continuously on the metric on $M$. For $C$ the dependence is with respect to the $W^{k+1, \infty}$-topology, and for $C^{\prime}$ the dependence is with respect to the $W^{k+2, \infty}$-topology on the space of metrics.

When restricted to functions such that $\int_{M} u=0$, the second estimate implies that the operator associated to the Neumann problem has bounded inverse.

\section{Orphaned lemmas and estimates}

Finally, we leave in this section a couple of estimates on Sobolev spaces which did not add to the presentation of the theory in chapter 1 , but are nonetheless useful. 
Lemma A.8 (Weh04, lemma B.3). Let $M$ be a compact Riemannian n-manifold, and let $k \in \mathbb{N}_{0}$ and $1 \leq p, r, s<\infty$ be such that either

$$
r, s \geq p \quad \text { and } \quad \frac{1}{r}+\frac{1}{s}<\frac{k}{n}+\frac{1}{p},
$$

or

$$
r, s>p \quad \text { and } \quad \frac{1}{r}+\frac{1}{s} \leq \frac{k}{n}+\frac{1}{p} .
$$

Then there is a constant $C$ such that for all $\alpha \in W^{k, r}(M)$ and $\beta \in W^{k, s}(M)$ the product lies in $W^{k, p}(M)$ and satisfies

$$
\|\alpha \cdot \beta\|_{k, p} \leq C\|\alpha\|_{k, r}\|\beta\|_{k, s} .
$$

A particular important case is $k \leq 1, r=s=p$ and $k p>n$.

Lemma A.9 (Weh04, lemma 6.5). Let $M$ be a compact Riemannian n-manifold and $1 \leq q \leq p<\infty$ such that $q \geq \frac{n}{2}$. In case $q<n$, assume further that $p \leq \frac{n q}{n-q}$. Then there exists a constant $C_{r n p q}$ such that, for all $f, g \in W^{1, p}(M)$

$$
\begin{aligned}
\|f \cdot g\|_{q} & \leq C_{r n p q}\|f\|_{r}\|g\|_{1, q}, \\
\|f \cdot g\|_{p} & \leq C_{r n p q}\|f\|_{r}\|g\|_{1, p}, \\
\|f \cdot g\|_{p} & \leq C_{r n p q}\|f\|_{1, q}\|g\|_{1, p},
\end{aligned}
$$

where we have:

(i) For $q<n, r=\frac{n q}{n-q} \geq p$,

(ii) for $q=n, r=2 p$,

(iii) and for $q>n, r=\infty$.

Whenever this lemma is used the constant will be called $C_{r n p q}$. The following lemma is sometimes called Kato's inequality, and we prove it in a particular case.

Lemma A.10. Let $E \rightarrow B$ be a vector bundle over $B \subseteq \mathbb{R}^{n}$ with the Euclidean metric $g_{i j}=\delta_{i j}$, and choose a metric and a compatible connection for $E$. Then for a section $A$ of $T^{*} M \otimes E$,

$$
|\nabla| A|| \leq|\nabla A|
$$

Proof. Write $A=A_{i} \mathrm{~d} x_{i}$, then

$$
(\nabla A)_{i j}=\nabla_{i} A\left(\partial_{j}\right)-A\left(\nabla_{i} \partial_{j}\right)=\nabla_{i} A_{j},
$$

because $\nabla_{i} \partial_{j}=\Gamma_{i j}^{k} \partial_{k}$ and the Christoffel symbols are zero for the Euclidean metric. Then simply write

$$
\nabla|A|=\mathrm{d}|A|=\frac{1}{2|A|} \mathrm{d}\langle A, A\rangle=\frac{1}{2|A|} \sum \partial_{i}\langle A, A\rangle \mathrm{d} x_{i}=\frac{1}{|A|} \sum\left\langle\nabla_{i} A, A\right\rangle \mathrm{d} x_{i},
$$

and use the Cauchy-Schwartz inequality in the following:

$$
|\nabla| A||^{2}=\sum \frac{\left\langle\nabla_{i} A, A\right\rangle^{2}}{|A|^{2}} \leq \sum \frac{\left|\nabla_{i} A\right|^{2}|A|^{2}}{|A|^{2}}=\sum\left|\nabla_{i} A\right|^{2}=|\nabla A|^{2} .
$$




\section{BIBLIOGRAPHY}

[AP95] Antonio Ambrosetti and Giovanni Prodi. A primer of nonlinear analysis, volume 34 of Cambridge Studies in Advanced Mathematics. Cambridge University Press, Cambridge, 1995. 64

[Aub82] Thierry Aubin. Nonlinear analysis on manifolds. Monge-Ampère equations, volume 252 of Grundlehren der Mathematischen Wissenschaften [Fundamental Principles of Mathematical Sciences]. Springer-Verlag, New York, 1982. 6, 19

[Cra15] Marius Crainic. Lecture notes for Mastermath course Differential Geometry 2015/2016. https://webspace.science.uu.nl/ crain101/DG-2015/main10. pdf, 2015. [Online; accessed 26-May-2021]. 1, 4, 5, 7, 53

[DK97] S. K. Donaldson and P. B. Kronheimer. The Geometry of Four-Manifolds. Oxford Mathematical Monographs. Clarendon Press, 1997. 25, 36, 37, 53

[Eva10] Lawrence C. Evans. Partial differential equations, volume 19 of Graduate Studies in Mathematics. American Mathematical Society, Providence, RI, second edition, 2010. 19

[FU91] D. Freed and K. Uhlenbeck. Instantons and Four-Manifolds. MSRI Publications. Springer-Verlag, 2 edition, 1991. 38

[GHL90] Sylvestre Gallot, Dominique Hulin, and Jacques Lafontaine. Riemannian geometry. Universitext. Springer-Verlag, Berlin, second edition, 1990. 18

[Hö7] Lars Hörmander. The analysis of linear partial differential operators. III. Classics in Mathematics. Springer, Berlin, 2007. Pseudo-differential operators, Reprint of the 1994 edition. 25

[Kna96] A. W. Knapp. Lie Groups Beyond an Introduction. Birkhäuser, 1996. 17

[Nic07] Liviu I. Nicolaescu. Lectures on the geometry of manifolds. World Scientific Publishing Co. Pte. Ltd., Hackensack, NJ, second edition, 2007. 19, 25

[Sch95] Günter Schwarz. Hodge decomposition - a method for solving boundary value problems, volume 1607 of Lecture Notes in Mathematics. Springer-Verlag, Berlin, 1995. 25

[Uhl82] Karen K. Uhlenbeck. Connections with $L^{p}$ bounds on curvature. Comm. Math. Phys., 83(1):31-42, 1982. 25, $39,52,57$ 
[War83] Frank W. Warner. Foundations of differentiable manifolds and Lie groups, volume 94 of Graduate Texts in Mathematics. Springer-Verlag, New York-Berlin, 1983. 37

[Weh04] K. Wehrheim. Uhlenbeck Compactness. Series of Lectures in Mathematics. EMS, 2004. 17, 19, 21, 22, 23, 24, 25, 30, 35, 39, 58, 63, 64, 65, 66

[Wel80] R. O. Wells. Differential analysis on complex manifolds. Graduate Texts in Mathematics. Springer-Verlag, 1980. 25 